\title{
Experimental:
}

All syntheses were carried out under deoxygenated and dried argon or nitrogen by use of standard Schlenk techniques or an inert gas glove box. All solvents were dried prior to use.

Elemental analyses were carried out using a Perkin Elmer Series II CHNS/O analyzer 2400 and a Perkin Elmer 2380 Atomic Absorption Spectrophotometer (Ni). NMR spectra were acquired on a Bruker Avance 400 spectrometer. IR UV/Vis spectra were acquired with a DU 650 Beckman spectrophotometer.

The ligands were synthesized according to modified literature methods $\left(\mathrm{Bz}_{3} \mathrm{TAC},(\mathrm{oFBz})_{3} \mathrm{TAC}\right)$.(see ref 1$)$

\section{Synthesis of hexakis(acetonitrile)nickeltetrafluoroborate}

$\left[(\mathrm{MeOH})_{6} \mathrm{Ni}\right]\left(\mathrm{BF}_{4}\right)_{2}$ was obtained according to the literature method [(a) Drago, R. S.; Meek, D. W.; Longhi, R.; Joesten, M. D. Inorg. Chem. 1963, 2, 1056-1060; (b) Wickenden, A. E.; Krause, R. A. Inorg. Chem. 1965, 4, 404-407.] by adding 2,2-dimethoxypropane ( $5 \mathrm{ml}, 40.7 \mathrm{mmol}$ ) (tri(ethyl)orthoformate can also be used) to hexaquonickeltetrafluoroborate $(1 \mathrm{~g}, 2.94 \mathrm{mmol})$ (recrystallized from saturated solution in thf, commercial or prepared from mixing $\mathrm{Ba}(\mathrm{OH})_{2}+2 \mathrm{HBF}_{4}(\mathrm{aq})+\mathrm{NiSO}_{4}(\mathrm{aq})$ after filtering off of $\left.\mathrm{BaSO}_{4}\right)$. The resulting green solution was stirred extensively for two hours. After removing the volatile materials an other $1 \mathrm{ml}$ of 2,2-dimethoxypropane was added, stirred for 15 minutes and then removed in vacuo. The raw $\left[(\mathrm{MeOH})_{6} \mathrm{Ni}\right]\left(\mathrm{BF}_{4}\right)_{2}$ was obtained as a light green solid in almost quantitative yield: Anal. Calcd for $\mathrm{C}_{6} \mathrm{H}_{24} \mathrm{O}_{6} \mathrm{~B}_{2} \mathrm{~F}_{8} \mathrm{Ni}: \mathrm{C}, 16.97 ; \mathrm{H}, 5.70$. Found: C, 15.80; H, 5.16.

Acetonitrile $(5 \mathrm{ml}, 95.7 \mathrm{mmol})$ was added to raw $\left[(\mathrm{MeOH})_{6} \mathrm{Ni}\right]\left(\mathrm{BF}_{4}\right)_{2}(1.25 \mathrm{~g}, 2.94 \mathrm{mmol})$ and stirred for 20 minutes. The volatile materials were removed in vacuo and the whole procedure was repeated two times. The blue $\left[(\mathrm{MeCN})_{6} \mathrm{Ni}\right]\left(\mathrm{BF}_{4}\right)_{2}$ was recrystallized by cooling of saturated solutions in acetonitrile. Anal. Calcd for $\mathrm{C}_{12} \mathrm{H}_{18} \mathrm{~N}_{6} \mathrm{~B}_{2} \mathrm{~F}_{8} \mathrm{Ni}(6 \mathrm{MeCN})$ : C, 30.11; H, 3.79; N, 17.56. Anal. Calcd for $\mathrm{C}_{10} \mathrm{H}_{15} \mathrm{~N}_{5} \mathrm{~B}_{2} \mathrm{~F}_{8} \mathrm{Ni}$ (5 MeCN): C, 27.45; H, 3.46; N, 16.01. Found: C, 28.1; H, 3.74; N, 16.0. Apparently up to one eq is lost during drying in vacuo.

$\left[(\mathrm{MeCN})_{4} \mathrm{Zn}\right]\left(\mathrm{BF}_{4}\right)_{2}$ was obtained in an analogous procedure resulting in colourless needles after recrystallisation from $\mathrm{MeCN}$ :

7.822 $\mathrm{g} \mathrm{Zn}\left(\mathrm{BF}_{4}\right)_{2}$ hydrate $\left(6-7 \mathrm{H}_{2} \mathrm{O}\right.$, ca $\left.22 \mathrm{mmol}\right)$ are dissolved in $15 \mathrm{ml} \mathrm{CH}(\mathrm{OEt})_{3}(90 \mathrm{mmol})$, then the solvent is slowly pumped off (mostly EtOH and $\mathrm{CHO}(\mathrm{OEt})_{3}$ ) under vacuum near RT. This was repeated 
three times by adding $8 \mathrm{ml} \mathrm{CH}(\mathrm{OEt})_{3}(3 \times 48 \mathrm{mmol})$ and pumping off the solvent. The residue becomes increasingly less soluble in added solvent. The final treatment with $\mathrm{CH}(\mathrm{OEt})_{3}$ was left over night and all solvent was pumped off to dryness. In the glove box, the solid was dissolved in $5 \mathrm{ml} \mathrm{MeCN}$ (ca $100 \mathrm{mmol}$ ), stirred for $1 \mathrm{~h}$, solvent pumped off, and the procedure repeated 3 times. Finally the solution was kept for 2 days, decanted from any insoluble precipitate and pumped dry again. The oily residue was washed with hexane and pumped dry several times until a nearly white solid remained. Yield 5.85g. Insufficient $\mathrm{EtOH} / \mathrm{MeCN}$ exchange may lead to material containing some EtOH. This can be used as well as EtOH is exchanged for the new ligand first.

Synthesis of 1a. $\mathrm{Bz}_{3}$ TAC $(3.20 \mathrm{mmol})$ was added to a solution of $\left[(\mathrm{MeCN})_{6} \mathrm{Ni}\right]\left(\mathrm{BF}_{4}\right)_{2}(3.20 \mathrm{mmol})$ in acetonitrile. The dark blue solution was stirred for half an hour. After removing all the volatile materials the crude product was washed three times with ether and dried in vacuo (90\% yield). Cooling of a solution in $\mathrm{MeCN}$, saturated at $40^{\circ} \mathrm{C}$, resulted in deep blue crystals. Alternatively, the original reaction solution can be layered with toluene or $\mathrm{Et}_{2} \mathrm{O}$ to give crystals of $\mathbf{1 a}$ (same cell constants). Anal. Calcd for $\mathrm{C}_{30} \mathrm{H}_{36} \mathrm{~N}_{6} \mathrm{NiB}_{2} \mathrm{~F}_{8}$ : C, 50.53; H, 5.09; N, 11.79; Ni, 8.23. Found: C, 50.01; H, 5.44; N, 11.96; Ni, 7.02. Mp $126^{\circ} \mathrm{C}$. UV/Vis $(\mathrm{MeCN}) v(\mathrm{~nm}) / \varepsilon\left(\mathrm{cm}^{-1} \cdot \mathrm{mol}^{-1} \cdot 1\right) 945 / 39,590 / 60,362 / 93,292 / 1261$.

1a/Zn, $1 \mathbf{b}$ and $\mathbf{1 b} / \mathbf{Z n}$ can be prepared analogously:

$100 \mathrm{mg}$ of $(\mathrm{oFPhCH} 2)_{3} \mathrm{TAC}(0.24 \mathrm{mmol})$ and $95 \mathrm{mg}$ of $\left[\mathrm{Ni}(\mathrm{MeCN})_{5}\right](\mathrm{BF} 4)_{2}(0.22 \mathrm{mmol})$ are dissolved in 1.3 $\mathrm{ml} \mathrm{MeCN}$ to give a deep blue 0.15 molar solution of $\mathbf{1 b}$ in $\mathrm{MeCN}$ (containing $10 \%$ free ligand to eliminate excess $\mathrm{Ni}$ ). Half of the solution is layered with $1 \mathrm{ml}$ of toluene. Blue crystals grow within a day. The excess solution is decanted and the crystals washed with $\mathrm{Et}_{2} \mathrm{O}$ to yield $64 \mathrm{mg}$ of $\mathbf{1 b}(75 \%)$.

The other half of the solution is used for NMR. A 0.09 molar solution with no excess ligand was obtained by use of slight excess of $\left[\mathrm{Ni}(\mathrm{MeCN})_{5}\right](\mathrm{BF} 4)_{2}\left(21.5 \mathrm{mg}\right.$ ligand with $26 \mathrm{mg}$ or $1.14 \mathrm{eq}$. of $\left[\mathrm{Ni}(\mathrm{MeCN})_{5}\right](\mathrm{BF} 4)_{2}$ in $0.53 \mathrm{ml}$ of $\mathrm{MeCN})$.

Anal. Calcd for $1 \mathbf{b}, \mathrm{C}_{30} \mathrm{H}_{33} \mathrm{~N}_{6} \mathrm{NiB}_{2} \mathrm{~F}_{11}$ : C, 46.98; H, 4.34; N, 10.96. Found: C, 47.1; H, 4.29; N, 10.9. $99 \mathrm{mg}$ of $\left(\mathrm{PhCH}_{2}\right)_{3} \mathrm{TAC}(0.277 \mathrm{mmol})$ and $80 \mathrm{mg}$ of $\left[\mathrm{Zn}(\mathrm{MeCN})_{4}\right](\mathrm{BF} 4)_{2}(0.20 \mathrm{mmol})$ are dissolved in 810 $\mathrm{mg} \mathrm{MeCN}$ to give a nearly colourless 0.16 molar solution of $\mathbf{1 a} / \mathbf{Z n}$ in $\mathrm{MeCN}$ (containing $40 \%$ excess 
$\left.\left(\mathrm{PhCH}_{2}\right)_{3} \mathrm{TAC}\right)$. The solution was layered with $1 \mathrm{ml}$ toluene and left standing in the glove box. Colourless crystals grow over night. The solvent was poured off, the residue washed with $\mathrm{Et}_{2} \mathrm{O}$ and dried in vacuo. $27.5 \mathrm{mg}$ of $\left(\mathrm{oFPhCH}_{2}\right)_{3} \mathrm{TAC}(0.091 \mathrm{mmol})$ and $42 \mathrm{mg}$ of $\left[\mathrm{Zn}(\mathrm{MeCN})_{4}\right](\mathrm{BF} 4)_{2}(0.104 \mathrm{mmol})$ are dissolved in $0.92 \mathrm{ml} \mathrm{MeCN}$ to give a slightly yellowish 0.09 molar solution of $\mathbf{1 b} / \mathbf{Z n}$ in $\mathrm{MeCN}$ (containing $10 \%$ excess $\left.\left[\mathrm{Zn}(\mathrm{MeCN})_{4}\right]^{2+}\right)$. Half of the solution is layered with $1 \mathrm{ml}$ of toluene. Colourless crystals grew within a day. The excess solution is decanted and the crystals washed with $\mathrm{Et}_{2} \mathrm{O}$, the other half of the solution is used for NMR. 


\section{X-Ray crystallography}

Intensity data for $\mathbf{1 a}, \mathbf{1 b}$ and $\mathbf{1 b} / \mathbf{Z n}$ were collected on a Nonius KappaCCD diffractometer equipped with a low temperature device, using graphite monochromated MoKa radiation $(\lambda=0.71070 \AA)$. Data were processed using the Nonius Software ${ }^{1}$. Crystal parameters and details on data collection, solution and refinement for the complexes are provided in Table 1. Structure solution, followed by full-matrix least squares refinement was performed using the WinGX-1.70 suite of programs throughout. ${ }^{2}$

Notes on the refinement: in $\mathbf{1 b}$ and $\mathbf{1 b} / \mathbf{Z n}$ the central $\mathrm{NCMe}$ and the two $\mathrm{BF}_{4}$ groups show a 1:1 disorder. $\mathrm{B} 1$ and $\mathrm{B} 1 \mathrm{~A}$ are refined isotropically. In 1a one of the phenyl rings of the ligand shows a 1:1 disorder while one of the two BF4 groups show rotational disorder in the ratio 70:30.

1. DENZO-SCALEPACK Z. Otwinowski and W. Minor, " Processing of X-ray Diffraction Data Collected in Oscillation Mode ", Methods in Enzymology, Volume 276: Macromolecular Crystallography, part A, p.307-326, 1997,C.W. Carter, Jr. \& R. M. Sweet, Eds., Academic Press.

2. L.J. Farrugia, J. Appl. Cryst., 1999, 32, 837-838.

Table 1. Crystal and structure refinement data for $\mathbf{1 a}, \mathbf{1 b}$ and $\mathbf{1 b} / \mathbf{Z n}$.

\begin{tabular}{|c|c|c|c|}
\hline Compound & $1 \mathrm{a}\left[\mathrm{BF}_{4}\right]_{2}$ & $1 \mathrm{~b}\left[\mathrm{BF}_{4}\right]_{2}$ & $1 \mathrm{~b} / \mathrm{Zn}\left[\mathrm{BF}_{4}\right]_{2}$ \\
\hline Empirical formula & $\mathrm{C}_{30} \mathrm{H}_{36} \mathrm{~B}_{2} \mathrm{~F}_{8} \mathrm{~N}_{6} \mathrm{Ni}$ & $\mathrm{C}_{30} \mathrm{H}_{33} \mathrm{~B}_{2} \mathrm{~F}_{11} \mathrm{~N}_{6} \mathrm{Ni}$ & $\mathrm{C}_{30} \mathrm{H}_{33} \mathrm{~B}_{2} \mathrm{~F}_{11} \mathrm{~N}_{6} \mathrm{Zn}$ \\
\hline Formula weight & 712.98 & 766.95 & 773.61 \\
\hline Temperature & $150(2)$ & $250(2)$ & $150(2)$ \\
\hline Wavelength / $\AA$ & 0.71073 & 0.71073 & 0.71073 \\
\hline Crystal system & Triclinic & Triclinic & Triclinic \\
\hline Space group & P-1 & $\mathrm{P}-1$ & $\mathrm{P}-1$ \\
\hline$a / \AA$ & $10.9500(2)$ & $10.8000(1)$ & $10.7380(2)$ \\
\hline$b / \AA$ & $10.9920(2)$ & $11.7640(1)$ & $11.7460(2)$ \\
\hline$c / \AA$ & $16.3930(4)$ & $14.7490(2)$ & $14.6580(2)$ \\
\hline$\alpha /{ }^{\circ}$ & $71.500(1)$ & $105.698(1)$ & $105.6230(6)$ \\
\hline$\beta / \circ$ & $71.165(1)$ & $91.382(1)$ & $91.1820(6)$ \\
\hline$\gamma / \circ$ & $71.625(1)$ & $100.045(1)$ & $100.0630(5)$ \\
\hline Volume / $\AA^{3}$ & $1720.45(6)$ & $1771.3(3)$ & $1748.62(5)$ \\
\hline $\mathrm{Z}$ & 2 & 2 & 2 \\
\hline Density (calculated) $/ \mathrm{mg} / \mathrm{m}^{3}$ & 1.376 & 1.438 & 1.469 \\
\hline Absorption coefficient $/ \mathrm{mm}^{-1}$ & 0.637 & 0.636 & 0.792 \\
\hline $\mathrm{F}(000)$ & 736 & 784 & 788 \\
\hline Crystal size/ mm & $0.45 \times 0.38 \times 0.15$ & $0.35 \times 0.25 \times 0.2$ & $0.5 \times 0.35 \times 0.3$ \\
\hline Theta range for data collection $/^{\circ}$ & 3.83 to 29.60 & 3.34 to 27.54 & 2.99 to 27.51 \\
\hline Reflections collected & 33304 & 31733 & 29102 \\
\hline Independent reflections & $9592[\mathrm{R}(\mathrm{int})=0.0564]$ & $8097[\mathrm{R}(\mathrm{int})=0.0285]$ & $7929[\mathrm{R}(\mathrm{int})=0.0367]$ \\
\hline Data/restraints/parameters & $9592 / 0 / 518$ & 8097 / 0 / 561 & 29102 / 0 / 561 \\
\hline Largest diff. peak \& hole/ e. $\AA^{-3}$ & 0.408 and -0.566 & 0.434 and -0.418 & 0.529 and -0.699 \\
\hline Final $R^{[a, b]}$ indices $[I>2 \sigma(I)]$ & $\begin{array}{l}\mathrm{R}_{1}=0.0433 \\
\mathrm{R}_{2}=0.0998\end{array}$ & $\begin{array}{l}\mathrm{R}_{1}=0.0392 \\
\mathrm{R}_{2}=0.1011\end{array}$ & $\begin{array}{l}\mathrm{R}_{1}=0.0405 \\
\mathrm{R}_{2}=0.1000\end{array}$ \\
\hline $\mathrm{R}^{[\mathrm{a}, \mathbf{b}]}$ indices (all data) & $\begin{array}{l}\mathrm{R}_{1}=0.0695 \\
\mathrm{wR}=0.01107\end{array}$ & $\begin{array}{l}\mathrm{R}_{1}=0.0461 \\
\mathrm{wR}_{2}=0.1055\end{array}$ & $\begin{array}{l}\mathrm{R}_{1}=0.0499 \\
\mathrm{wR}_{2}=0.1057\end{array}$ \\
\hline Goodness-of-fit on $\mathrm{F}^{2[\mathrm{c}]}$ & 1.023 & 1.029 & 1.030 \\
\hline
\end{tabular}




\section{NMR Spectroscopy}

NMR spectra were obtained in acetonitrile with a $\mathrm{D}_{2} \mathrm{O}$ capillary for lock and reference. Chemical shifts are reported relative to the acetonitrile solvent signal $\left(1.96 \mathrm{ppm}\right.$ for ${ }^{1} \mathrm{H}$ and $1.79 \mathrm{ppm}$ for $\left.{ }^{13} \mathrm{C}\right)$. The ${ }^{1} \mathrm{H}$ chemical shift of neat acetonitrile against HDO (4.70ppm) in the capillary was $2.73 \mathrm{ppm}$ and was used to determine the bulk susceptibility shift for the Evans method and to correct the observed ${ }^{19} \mathrm{~F}$ shifts. Spectra with excess ligand as internal reference confirmed the validity of this external correction method. NMR spectra were obtained for concentrated solution (0.1M) in MeCN. More dilute solutions give nearly the same chemical shifts and relative line widths at the expense of diminished signal/noise ratios. Diamagnetic reference spectra are obtained from the analogous zinc complexes.

Spectra of the paramagnetic nickel complexes at appropriate repetition times according to the widths of the observed signals (from 40ms acquisition time AQ, 10ms delay D1 for 1500ppm window (SW) for 200,000 scans (NS) for broad ${ }^{13} \mathrm{C}$ signals to $1 \mathrm{~s}(\mathrm{AQ}+\mathrm{D} 1)$, 40ppm SW and 128 scans for narrow aromatic ${ }^{1} \mathrm{H}$ and ${ }^{13} \mathrm{C}$ signals. $\mathrm{T}_{1}$ (inversion recovery) and $\mathrm{T}_{2}$ (Carr-Purcell-Meiboom-Gill, cpmg) measurements were obtained by measuring several windows of 50ppm or less to ensure good $90 / 180^{\circ}$ pulses and $10-30$ variable delays for three parameter fits. As $\mathrm{T}_{1}$ is much shorter than the $\mathrm{C}-\mathrm{H}$ cross relaxation in most cases, ${ }^{1} \mathrm{H}$ decoupling was found to be unnecessary except for the narrowest ${ }^{13} \mathrm{C}$ signals. $\mathrm{T}_{2}$ was mostly obtained from the line widths and agreed well with the cpmg measurements done for some peaks.

Assignment of the signals is based on integration, linewidths, ${ }^{2} \mathrm{H}$ NMR signals observed for selectively deuterated 1a (ring $\mathrm{CD}_{2}$ at +28 and $+9 \mathrm{ppm}$, benzylic $\mathrm{CD}_{2}$ at $+150 \mathrm{ppm}$ and $\mathrm{CD}_{3} \mathrm{CN}$ at $-30 \mathrm{ppm}$ when measured in $\mathrm{CD}_{3} \mathrm{NO}_{2}$, details to be included in a future publication), and the reduction of linewidths for broad ${ }^{13} \mathrm{C}$ signals upon selective decoupling of the attached ${ }^{1} \mathrm{H}$ signal. The ring $\mathrm{H}$ signals were assigned based on selective NOEs on the two doublets: NOE at ortho $\mathrm{CH}$ is 4 times and at benzylic $\mathrm{CH}_{2} 2$ times larger when irradiated at $4 \mathrm{ppm}$ then when irradiated at $3.4 \mathrm{ppm}$ (relative to NOE between the two ring signals).

Line widths were determined by Lorentz curve fitting after manual polynomial baseline correction provided by Bruker xwin-nmr, or in the case of broad and isolated peaks by a six-parameter fit for a Lorentz shape curve plus a second order polynomial baseline using ASCII data in ORIGIN (Microcal Origin 6.0). The latter procedure eliminates most of the subjective effects of the manual baseline correction and gave more reliable values. All line shape fits were performed by ORIGIN after conversion of the Bruker NMR spectra to ASCII format.

The measured relaxation times (but not the shifts) are generally increasing upon dilution (about 10\% on 4-fold dilution) due to reduced viscosity and outer sphere relaxation due to neighbouring paramagnetic centers. The effect is much larger for diamagnetic signals and the $\mathrm{BF}_{4}$ anion. The latter indicates increased ion separation on dilution. However, the relative relaxation times within a complex do not change significantly - thus, the data are suitable for structural analysis.

As a diamagnetic reference for the coupling constant in coordinated $\mathrm{MeCN}$ commercial $\left[\mathrm{Pd}(\mathrm{MeCN})_{4}\right]\left(\mathrm{BF}_{4}\right)_{2}$ in $\mathrm{CD}_{3} \mathrm{NO}_{2}$ gave $139.8 \mathrm{~Hz}$, close to free $\mathrm{MeCN}(136 \mathrm{~Hz})$. 


\section{NMR Data}

Table 2. Data are given in the following format and are compared to values obtained from the line shape fit to the full expression for $T_{1}$ spin decoupling in red.

Chemical shift in ppm, multiplicity

$\mathrm{J}=$...coupling constant in $\mathrm{Hz}$ (suffix $\mathrm{F}$ for coupling to $\mathrm{F}$ ) [ $\mathrm{J}$ obtain from line shape fit] (Lorentz) line width $\mathrm{W}$, [W in ${ }^{13} \mathrm{C}\left\{{ }^{1} \mathrm{H}\right\}$ at region of attached $\left.\mathrm{H}\right]$

$\mathrm{T}_{2}{ }^{-1}$ as $\pi \mathrm{W}\left({ }^{13} \mathrm{C}\left\{{ }^{1} \mathrm{H}\right\}\right)$, from cpmg [from line shape fit]

$\mathrm{T}_{1}{ }^{-1}$ from inversion recovery [from ${ }^{13} \mathrm{C}$ line shape for ${ }^{1} \mathrm{H}$, or under $\mathrm{CH}$ decoupling for ${ }^{13} \mathrm{C}$ ]

\begin{tabular}{|c|c|c|c|c|}
\hline & $1 \mathbf{a}$ & 1a/Zn & $1 b$ & 1b/Zn \\
\hline $\mathrm{H}_{\mathrm{ax} \text {,ring }}$ & $\begin{array}{l}+28.1 \\
1263(3) \\
3970(10) \\
3450(230)\end{array}$ & $\begin{array}{l}3.25 \mathrm{~d} \\
\mathrm{~J}=9.0\end{array}$ & $\begin{array}{l}+26 \\
1256(4) \\
3950(10), 3710(110) \\
2970(90)\end{array}$ & $\begin{array}{l}3.36 \mathrm{~d} \\
\mathrm{~J}=9.0\end{array}$ \\
\hline $\mathrm{H}_{\text {eq,ring }}$ & $+9 *$ & $\begin{array}{l}4.00 \mathrm{~d} \\
\mathrm{~J}=9.0\end{array}$ & na & $\begin{array}{l}4.00 \mathrm{~d} \\
\mathrm{~J}=9.0\end{array}$ \\
\hline $\mathrm{C}_{\text {ring }}$ & na & $\begin{array}{l}72.7 t \\
J=153\end{array}$ & na & $\begin{array}{l}72.5 \mathrm{t} \\
\mathrm{J}=155\end{array}$ \\
\hline $\mathrm{H}_{\text {benzl }}$ & $\begin{array}{l}+141.8 \\
4604(6) \\
14460(20) \\
2750(100)\end{array}$ & $\begin{array}{l}3.80 \mathrm{~s} \\
10\end{array}$ & $\begin{array}{l}+142 \\
4600(10) \\
14450(40) \\
2760(70)\end{array}$ & $3.89 \mathrm{~s}$ \\
\hline $\mathrm{C}_{\text {benzyl }}$ & $\begin{array}{l}-88.6 \\
434(5)[396(5)] \\
1244(16) \\
534(48) \\
\end{array}$ & $\begin{array}{l}56.3 t \\
J=139\end{array}$ & $\begin{array}{l}-101 \\
410(10)[389(6)] \\
1220(20) \\
444(24)\end{array}$ & $\begin{array}{l}49.4 t \\
J=139 \\
J_{F}=2.8\end{array}$ \\
\hline $\mathrm{C}_{\mathrm{Ph} \text {,ipso }}$ & $\begin{array}{l}+441 \\
1191(11) \\
3740(40) \\
215(42) \\
\end{array}$ & $\begin{array}{l}134.0 \mathrm{t} \\
\mathrm{J}=6\end{array}$ & $\begin{array}{l}+420 \\
1040(30) \\
3270(90) \\
174(18) \\
\end{array}$ & $\begin{array}{l}121.2 \mathrm{~s} \\
\mathrm{~J}_{\mathrm{F}}=15.4\end{array}$ \\
\hline $\mathrm{H}_{\mathrm{Ph} \text {,ortho }}$ & $\begin{array}{l}+6.94 \\
357(34) \\
1120(100) \\
870(90)[900(60)]\end{array}$ & $7.4 \mathrm{~m}$ & $\begin{array}{l}7.40 \\
517(8) \\
1620(30) \\
1030(220)[1000(80)]\end{array}$ & $7.37 \mathrm{~m}$ \\
\hline $\mathrm{F}_{\mathrm{Ph} \text {,ortho }}$ & - & - & $\begin{array}{l}-114 \\
282(2) \\
886(7) \\
708(1)[724(16)]\end{array}$ & $\begin{array}{l}-117 \\
\mathrm{~J}_{\mathrm{C}}=248.9 \\
17(2)\end{array}$ \\
\hline $\mathrm{F}$ of $\mathrm{BF}_{4}$ & $\begin{array}{l}-151 \\
54.5(1) \\
171(1) \\
89.4(2)\end{array}$ & & $\begin{array}{l}-151 \\
53(1) \\
165(1) \\
99(1)\end{array}$ & $\begin{array}{l}-151 \\
2.2(1)\end{array}$ \\
\hline
\end{tabular}




\begin{tabular}{|c|c|c|c|c|}
\hline $\mathrm{C}_{\mathrm{Ph} \text {,ortho }}$ & $\begin{array}{l}138.0 \\
\mathrm{~J}=-[151(9)] \\
115(1)[25(1)] \\
79(4),[79(16)] \\
59(4)[48(6)]\end{array}$ & $\begin{array}{l}129.7 \mathrm{dd} \\
\mathrm{J}=7,161\end{array}$ & $\begin{array}{l}140.0 \\
\mathrm{~J}=-[156(12)] \\
95(7)[29(1)] \\
90(3), 61(6)[91(20)] \\
56(20)[51(3)] \\
\quad \text { and } \\
162.8 \mathrm{~d} \\
\mathrm{~J}=” 169(2) ”[249(3)] \\
\text { “135(3)" } \\
-, 53(4)[66(10)] \\
50(3)[44(4)]\end{array}$ & $\begin{array}{l}132.5 \mathrm{~d} \\
\mathrm{~J}=165 \\
\text { and } \\
162.5 \mathrm{~d} \\
\mathrm{~J}_{\mathrm{F}}=249\end{array}$ \\
\hline $\mathrm{H}_{\mathrm{Ph}, \text { meta }}$ & $\begin{array}{l}+8.86 \\
53(2) \\
167(7) \\
114(12)[130(9)]\end{array}$ & $7.3 \mathrm{~m}$ & $\begin{array}{l}8.72 \\
66(1) \\
207(4), 162(3) \\
109(12)[157(7)] \\
\text { and } \\
8.56 \\
70(1) \\
220(4) \\
126(12)[175(6)]\end{array}$ & $\begin{array}{l}7.23 \mathrm{~m} \\
\\
\text { and } \\
7.17 \mathrm{~m}\end{array}$ \\
\hline $\mathrm{C}_{\mathrm{Ph}, \text { meta }}$ & $\begin{array}{l}129.0 \mathrm{~d} \\
\mathrm{~J}=149[160(1)] \\
\text { “35(1)" }[12(2)] \\
38(7)[38(6)] \\
18(1)[14.7(2.4)]\end{array}$ & $\begin{array}{l}131.9 t d \\
J=6,159\end{array}$ & $\begin{array}{l}114.5 \mathrm{~d} \\
\mathrm{~J}=156[166(1)] \\
\text { “32(10)”[15] } \\
-, 23(2)[20(5)] \\
18(2)[16(2)] \\
\text { And } \\
125.8 \mathrm{~d} \\
\mathrm{~J}=156,[166(1)] \\
\text { “54(14)” }[10] \\
-, 20(2)[9(4)] \\
18(2)[16(2)]\end{array}$ & $\begin{array}{l}116.7 \mathrm{dd} \\
\mathrm{J}=168 \\
\mathrm{~J}_{\mathrm{F}}=24 \\
\\
\text { And } \\
125.8 \mathrm{~d} \\
\mathrm{~J}=164\end{array}$ \\
\hline $\mathrm{H}_{\mathrm{Ph} \text {,para }}$ & $\begin{array}{l}+5.80 \\
32(2) \\
101(7) \\
61(7)[71(7)] \\
\end{array}$ & $7.4 \mathrm{~m}$ & $\begin{array}{l}+5.95 \\
33(1) \\
104(4), 93(2) \\
67(7)[65(12)]\end{array}$ & $7.4 \mathrm{~m}$ \\
\hline $\mathrm{C}_{\mathrm{Ph} \text {,para }}$ & $\begin{array}{l}139.5 \mathrm{~d} \\
\mathrm{~J}=159[160(1)] \\
\text { “23(1)” }[11(1)] \\
35(4)[35(5)] \\
12(1)[9.4(1.7)]\end{array}$ & $\begin{array}{l}129.8 \mathrm{td} \\
\mathrm{J}=7,160\end{array}$ & $\begin{array}{l}+141.9 \mathrm{~d} \\
\mathrm{~J}=161[163(7)] \\
21(1)[11(1)] \\
35(4), 19(2)[32(9)] \\
9(2)[10(1)]\end{array}$ & $\begin{array}{l}134.5 d \\
J=159\end{array}$ \\
\hline $\mathrm{H}_{M e \mathrm{CN}}$ & $\begin{array}{l}-32.6 \\
284(1) \\
892(1) \\
327(6) \\
\end{array}$ & na & $\begin{array}{l}-32.2 \\
271(1) \\
850(4), 930(60) \\
330(30) \\
\end{array}$ & na \\
\hline $\mathrm{C}_{\underline{\underline{M e}} \mathrm{CN}}$ & $\begin{array}{l}+205 \\
473(3)[337(2)] \\
1059(7) \\
77(9)\end{array}$ & na & $\begin{array}{l}+204 \\
414(5)[314(10)] \\
986(30), 910(90) \\
74(7)\end{array}$ & na \\
\hline
\end{tabular}

* shift obtained from ${ }^{2} \mathrm{H}$ NMR of the ring deuterated $1\left(\mathrm{H}_{\mathrm{ax}}\right.$ at $\left.28.8 \mathrm{ppm}\right)$ (widths 44 for $\mathrm{H}_{\mathrm{ax}}$ and $91 \mathrm{~Hz}$ for $\mathrm{H}_{\mathrm{eq}}$ ) 


\section{${ }^{1} \mathrm{H}$ (top) and ${ }^{13} \mathrm{C}$ (bottom) NMR spectra of $\mathbf{1 a}$ in $\mathrm{MeCN}$}

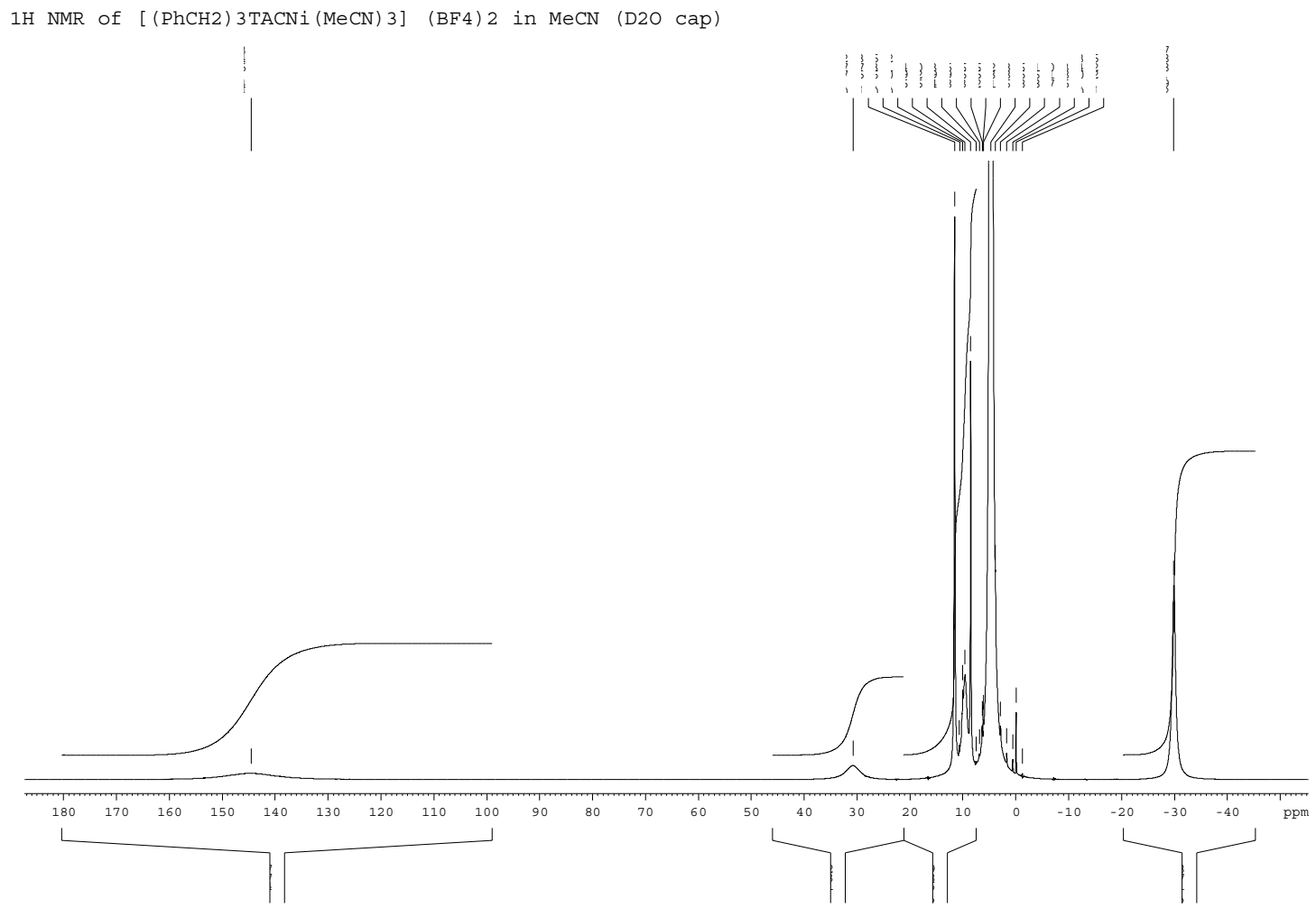

13C of [Bz3TACNi (MeCN) 3] (BF4) 2 in MeCN (D2O cap) decoupled at $140 \mathrm{ppm}$

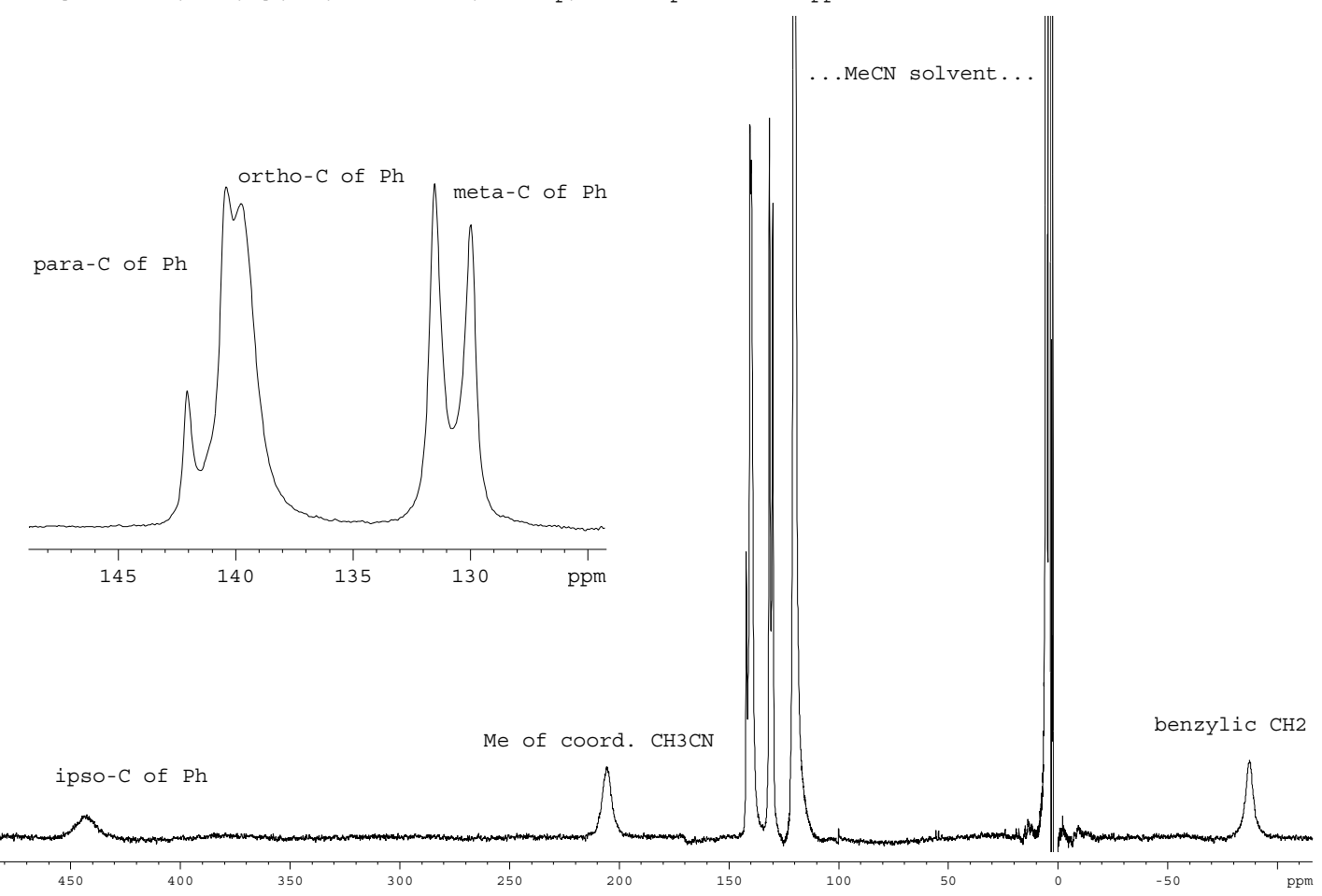


Comparison of coupled versus decoupled ${ }^{13} \mathrm{C}$ NMR of $\mathbf{1 a}$ in the aromatic region. Note that the ortho-CH signal remains a single peak without $\left\{{ }^{1} \mathrm{H}\right\}$ decoupling. It is broadened but not broader the expected $\mathrm{J}(\mathrm{C}-\mathrm{H})$ coupling as seen for the signals for meta- and para$\mathrm{CH}$.

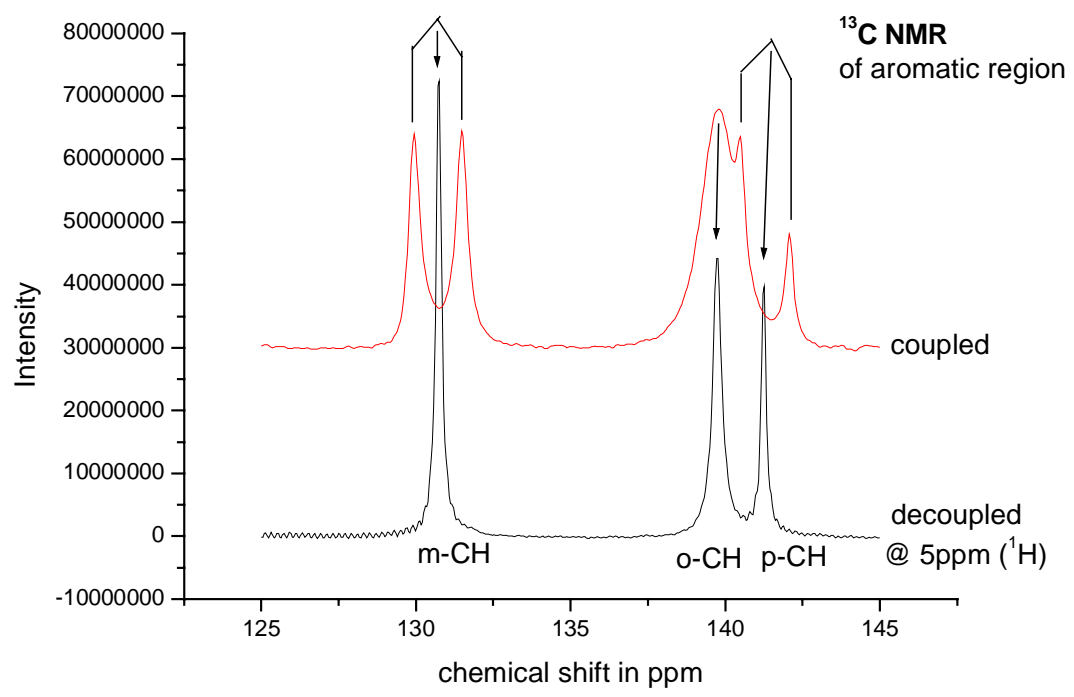

10-Parameter fit with equation eqS1 (5 parameter for each peak) for the ortho- and para$\mathrm{CH}$ signals in 1a. Minimum $\mathrm{T}_{2 \mathrm{~A}}{ }^{-1}$ set to experimental $\pi \mathrm{W}$ of the decoupled spectrum. (Arbitrary intensity units)

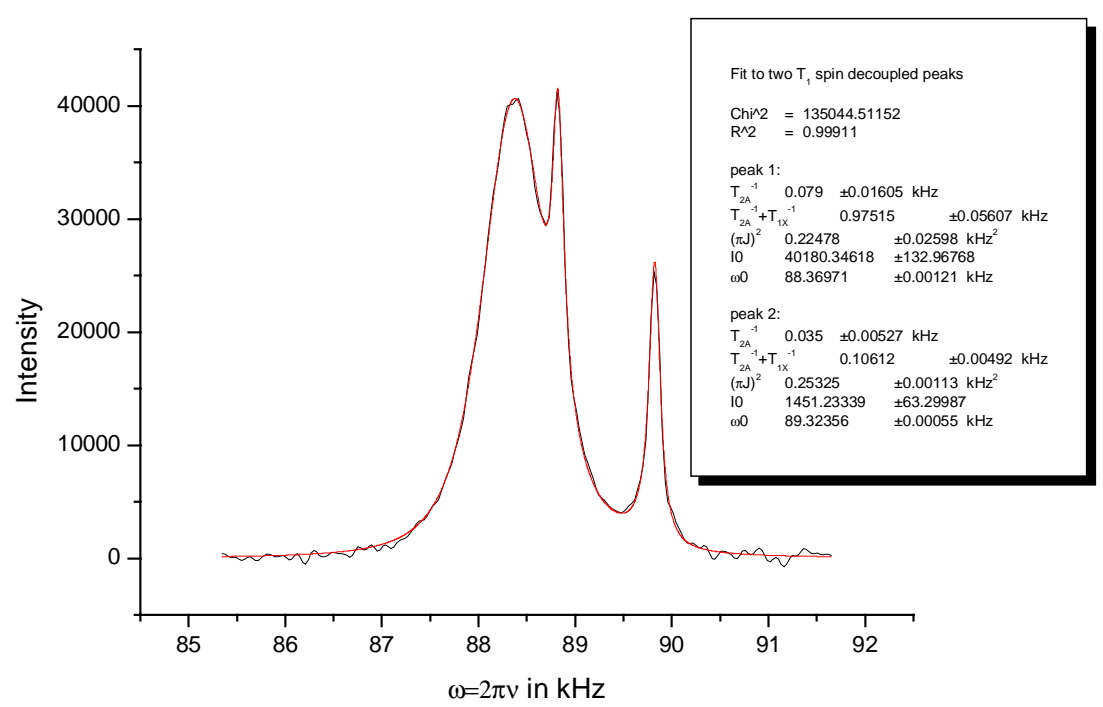

$\delta=139.77-1.73=138.04 \mathrm{ppm}, \mathrm{W}=25(5) \mathrm{Hz}, \mathrm{T}_{1 \mathrm{X}}{ }^{-1}=896(58) \mathrm{Hz}, \mathrm{J}=151(9) \mathrm{Hz}$

$\delta=141.28-1.73=139.55 \mathrm{ppm}, \mathrm{W}=11(2) \mathrm{Hz}, \mathrm{T}_{1 \mathrm{X}}{ }^{-1}=71(7) \mathrm{Hz}, \mathrm{J}=160.2(9) \mathrm{Hz}$ 
Coupled ${ }^{13} \mathrm{C}$ NMR signal for the aromatic meta-CH in $\mathbf{1 a}$ with the best fit for two Lorentz-shaped peaks of equal area and width (doublet).

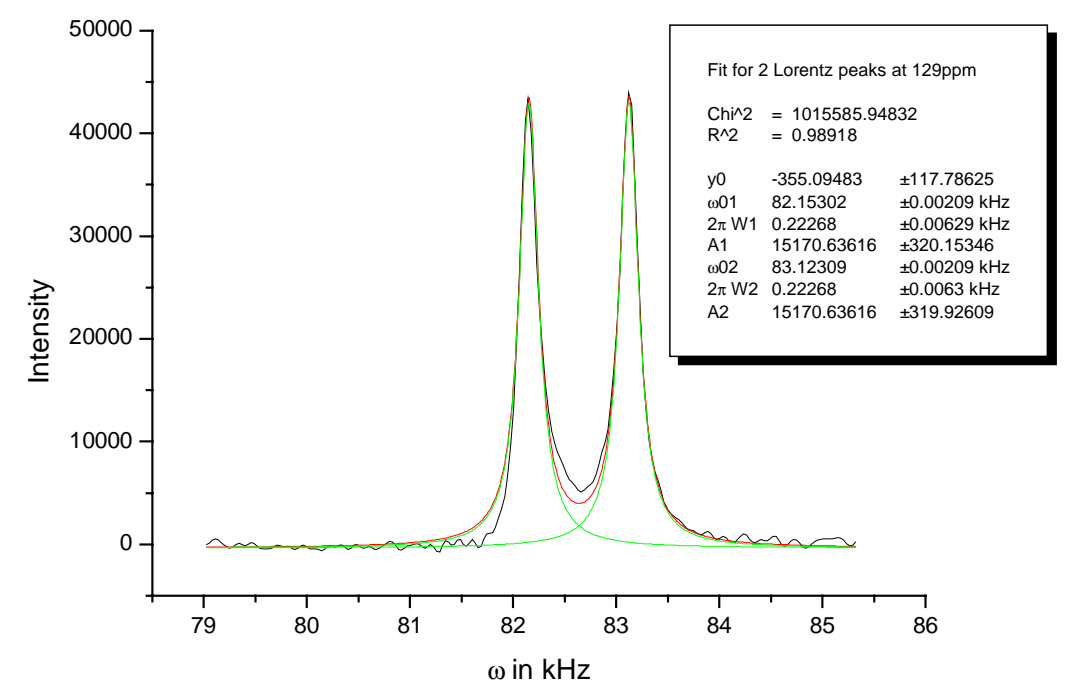

$\delta=130.71-1.73=128.98 \mathrm{ppm}, \mathrm{W}=35.4(1.0) \mathrm{Hz},,{ }^{\prime \prime} “=154(2) \mathrm{Hz}$

The fit is poor in the region between the two peaks.

Coupled ${ }^{13} \mathrm{C}$ NMR signal for the aromatic meta-CH in $1 \mathrm{a}$ with the best 5-parameter fit using equation eqS1.

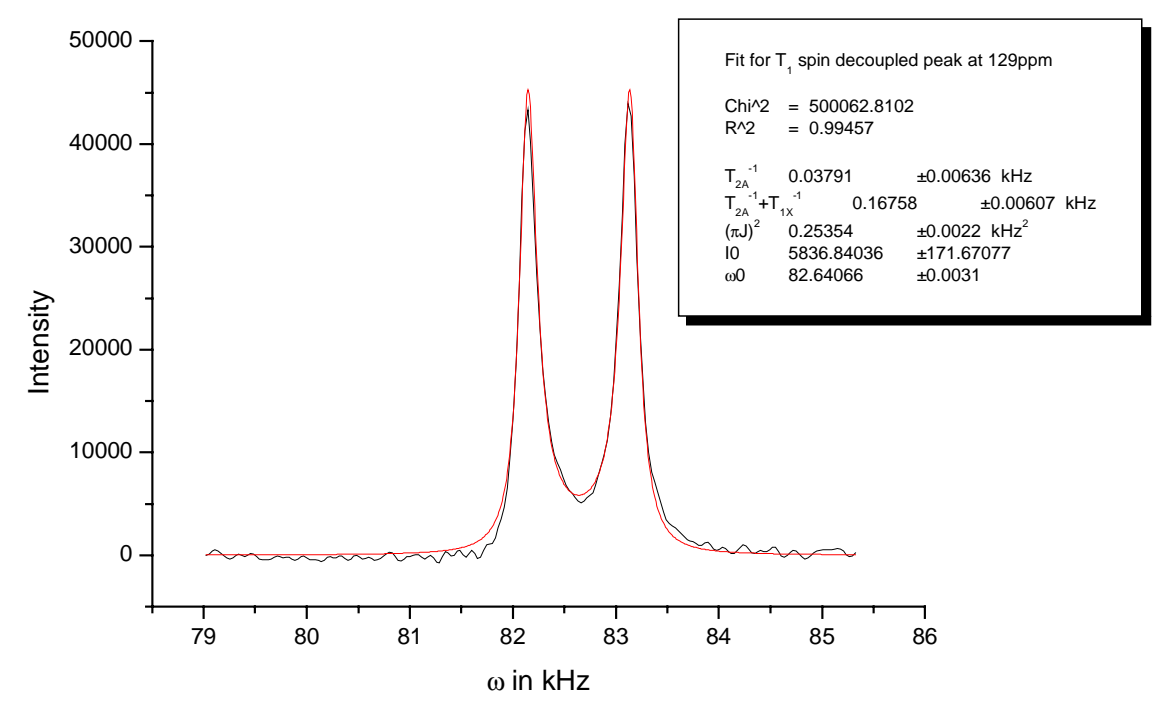

$\delta=130.71-1.73=128.98 \mathrm{ppm}, \mathrm{W}=12.0(2.0) \mathrm{Hz}, \mathrm{T}_{1 \mathrm{X}}{ }^{-1}=130(9) \mathrm{Hz}, \mathrm{J}=160.3(10) \mathrm{Hz}$

The fit is much better than for a Lorentz doublet! 
Effect of decoupling on ${ }^{13} \mathrm{C}$ NMR peaks much broader than the expected coupling constant: coordinated $\underline{\mathrm{MeCN}}$ (top) and benzylic $\underline{\mathrm{CH}} \mathrm{H}_{2}$ (bottom) in 1a
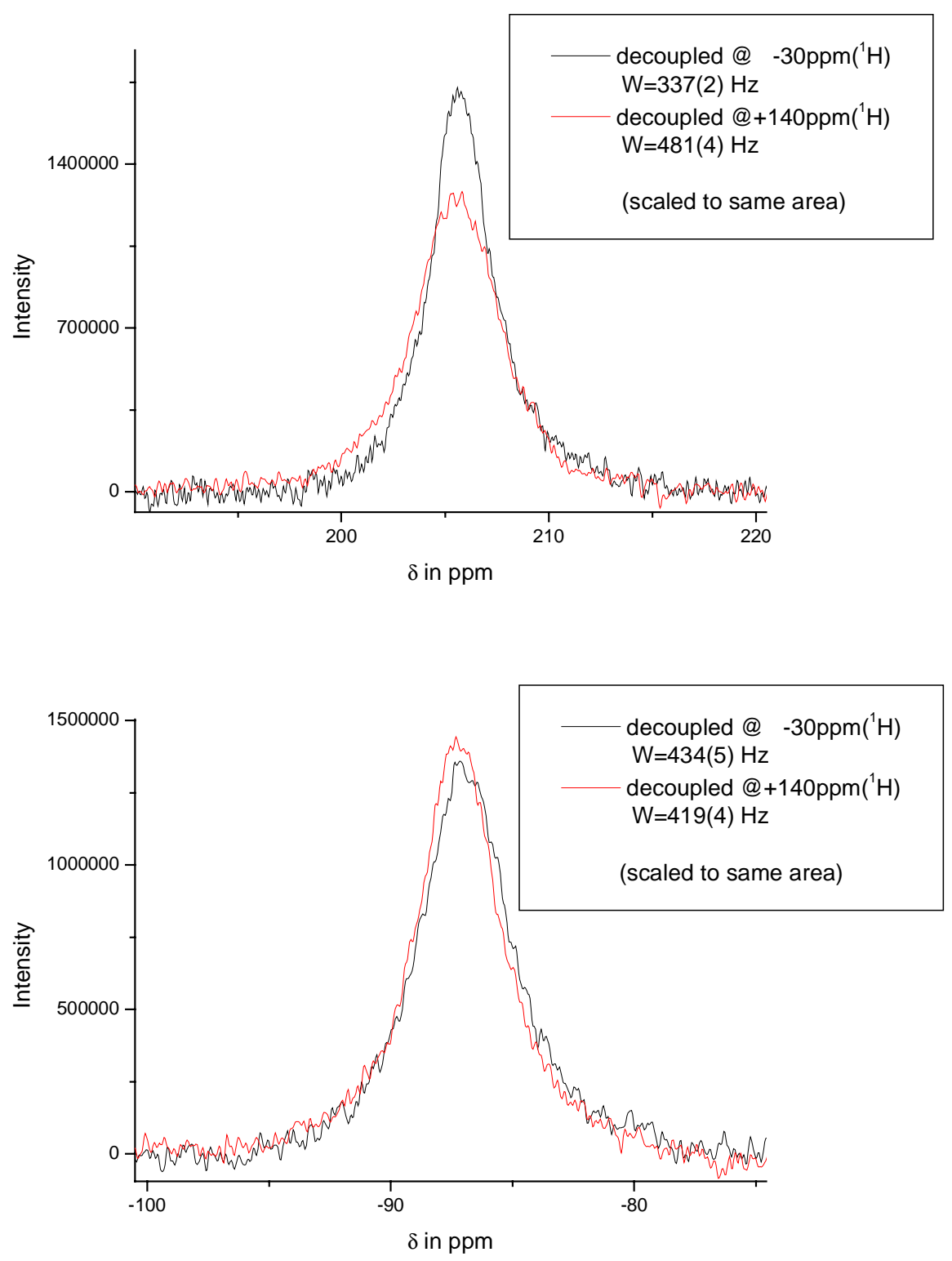

Comparison of the spectra with $\left\{{ }^{1} \mathrm{H}\right\}$ decoupling at $-30 \mathrm{ppm}$ in ${ }^{1} \mathrm{H}$ (black) and at $+140 \mathrm{ppm}$ (red):

The peak at $+205 \mathrm{ppm}$ becomes much narrower by decoupling at $-30 \mathrm{ppm}\left(\mathrm{C}_{3} \mathrm{CN}-\mathrm{Ni}\right)$ The peak at $-88 \mathrm{ppm}$ becomes narrower by decoupling at $-140 \mathrm{ppm}\left(\mathrm{NC}_{2} \mathrm{Ph}\right)$ 
Spectra of $\mathbf{1 b}$ in $\mathrm{MeCN}\left(10 \% \mathrm{Et}_{2} \mathrm{O}\right.$ added as diamagnetic reference)

\section{${ }^{1} \mathrm{H}$ NMR}

$0.1 \mathrm{M}$ [ (oFBz3TAC) Ni (MeCN) 3] (BF4) 2 in MeCN (10\% Et20)

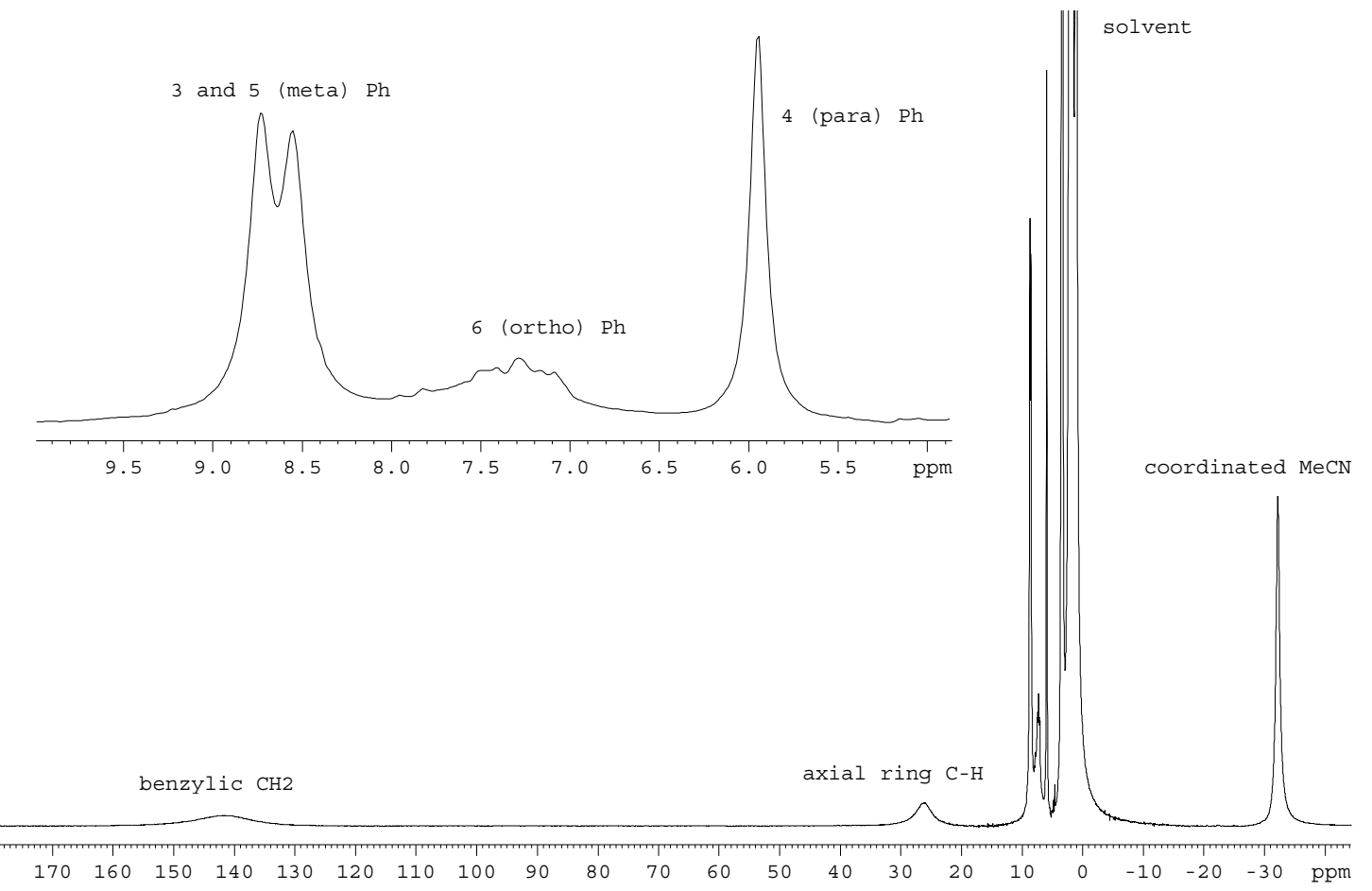

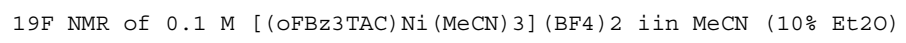

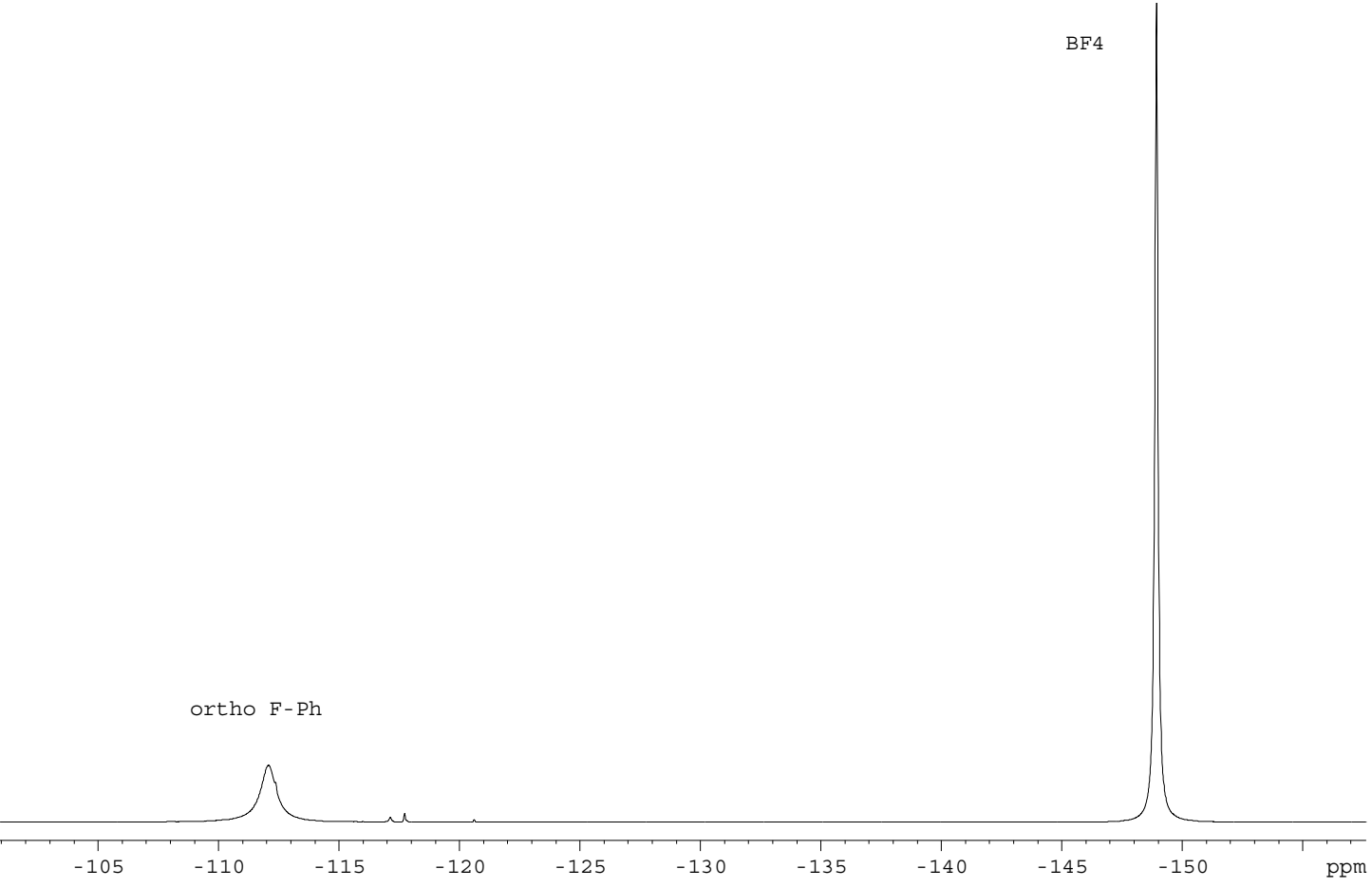


$13 \mathrm{C}\{1 \mathrm{H}$ at $146 \mathrm{ppm}\}$ of $[(\mathrm{OFBz} 3 \mathrm{TAC}) \mathrm{Ni}(\mathrm{MeCN}) 3](\mathrm{BF} 4) 2$ in $\mathrm{MeCN}$ (10\% Et2O)

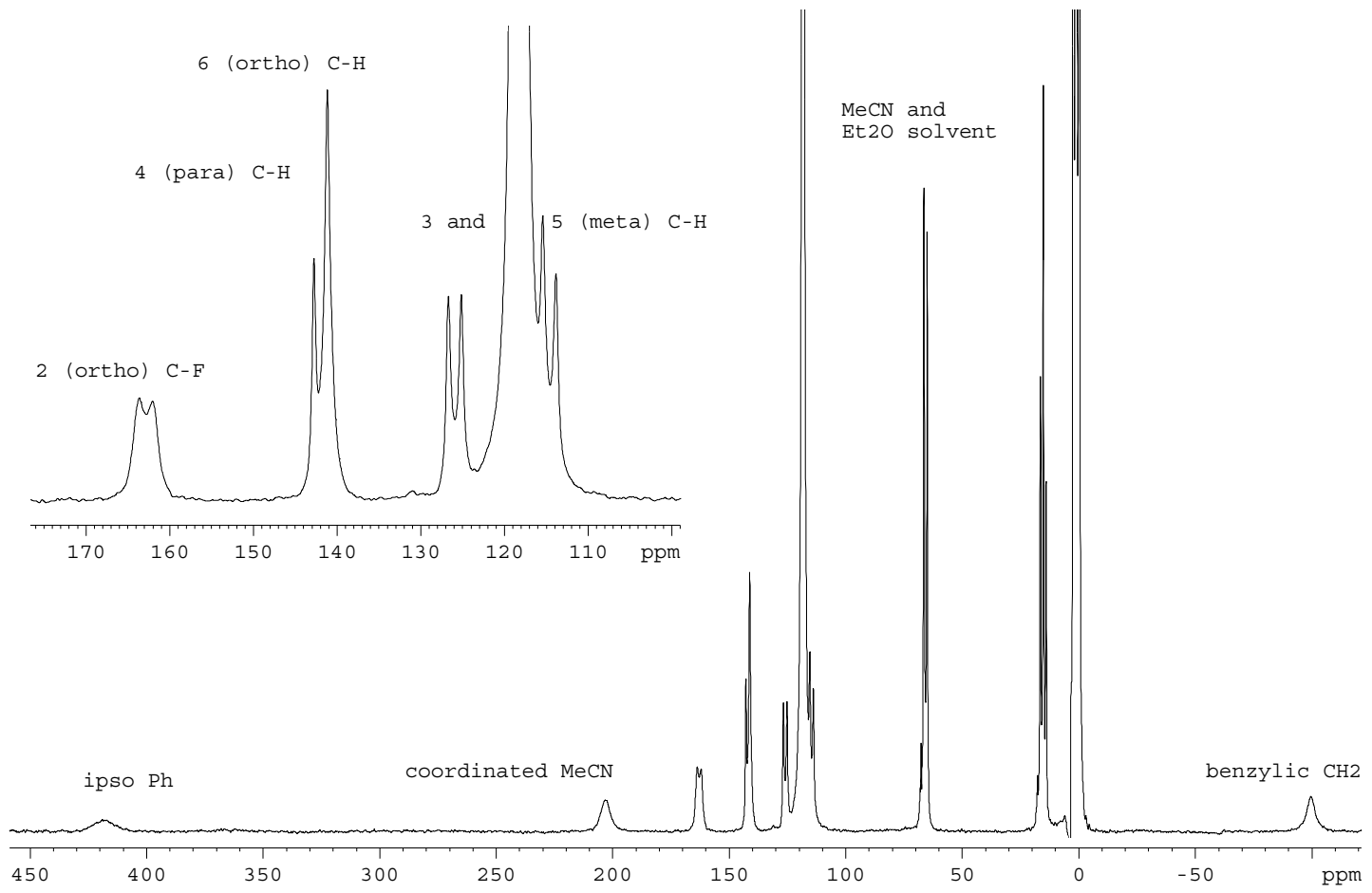

$13 \mathrm{C}\{1 \mathrm{H}$ at $7 \mathrm{ppm}\}$ of $[(\mathrm{OFBz} 3 \mathrm{TAC}) \mathrm{Ni}(\mathrm{MeCN}) 3](\mathrm{BF} 4) 2$ in $\mathrm{MeCN}$ (10\% Et 2O)

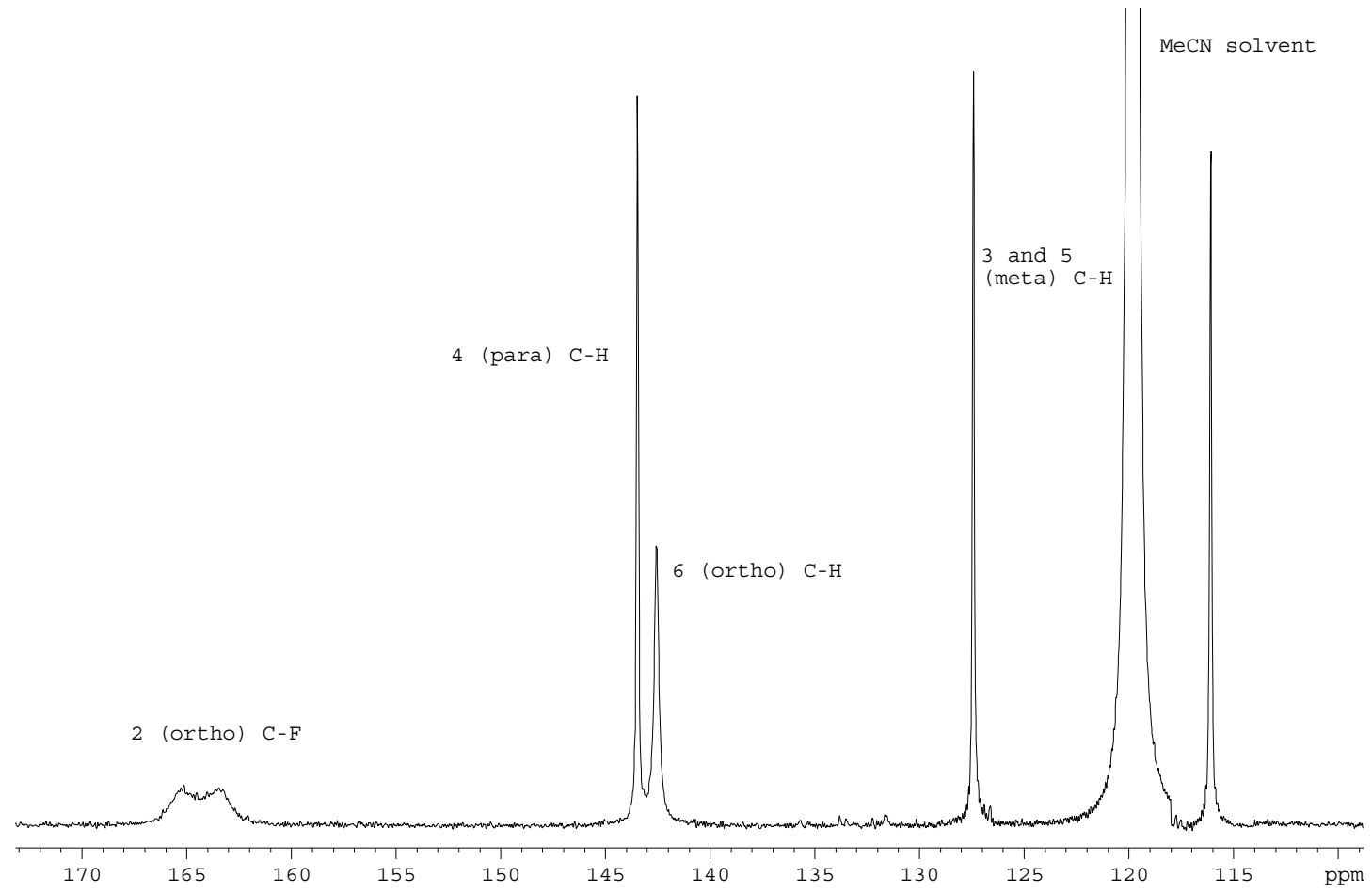


NMR spectra of the Zn analogue of $\mathbf{1 b}: \mathbf{1 b} / \mathbf{Z n}$

\section{${ }^{1}$ H NMR (400 MHz)}

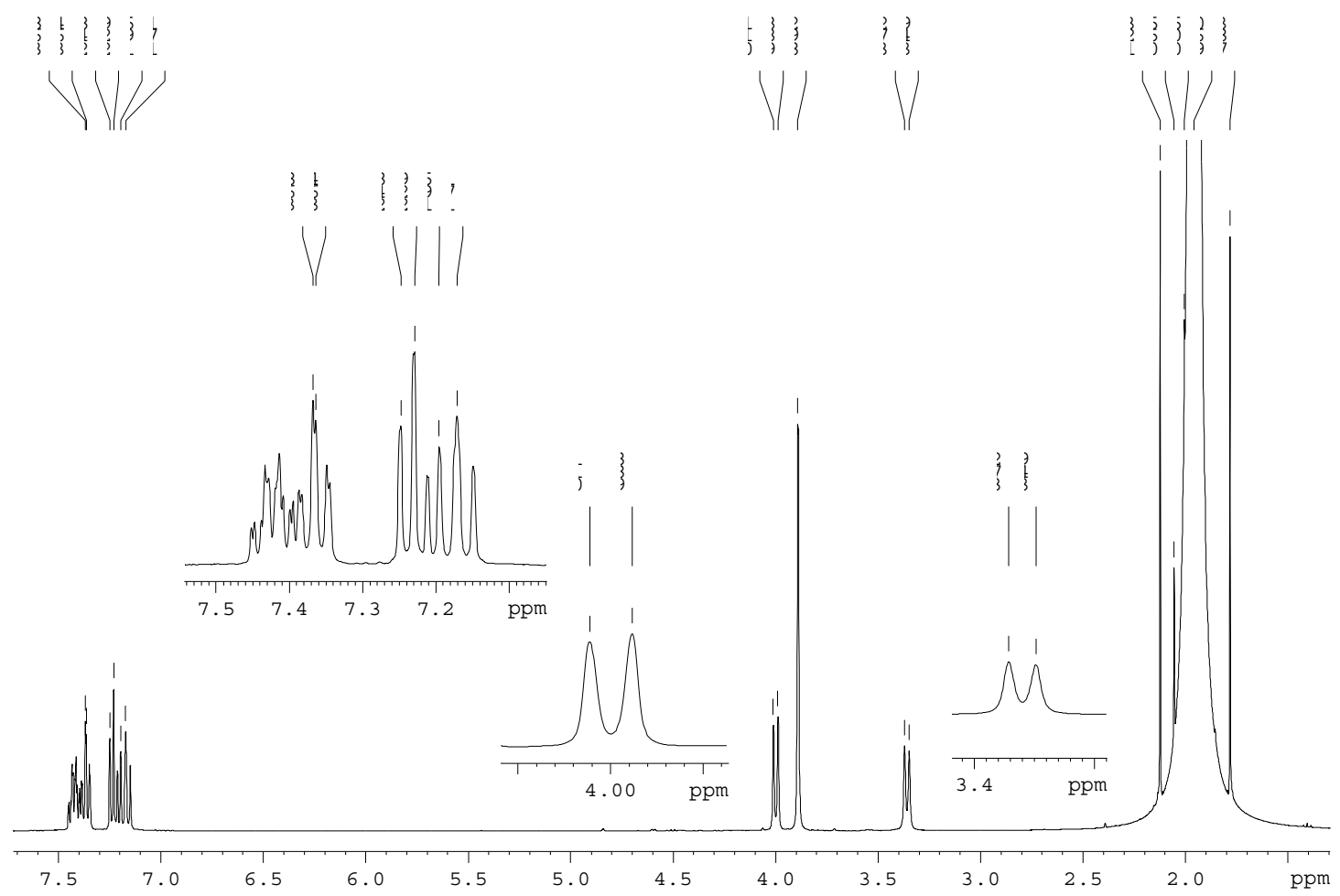

\section{${ }^{19}$ F NMR (376 MHz)}

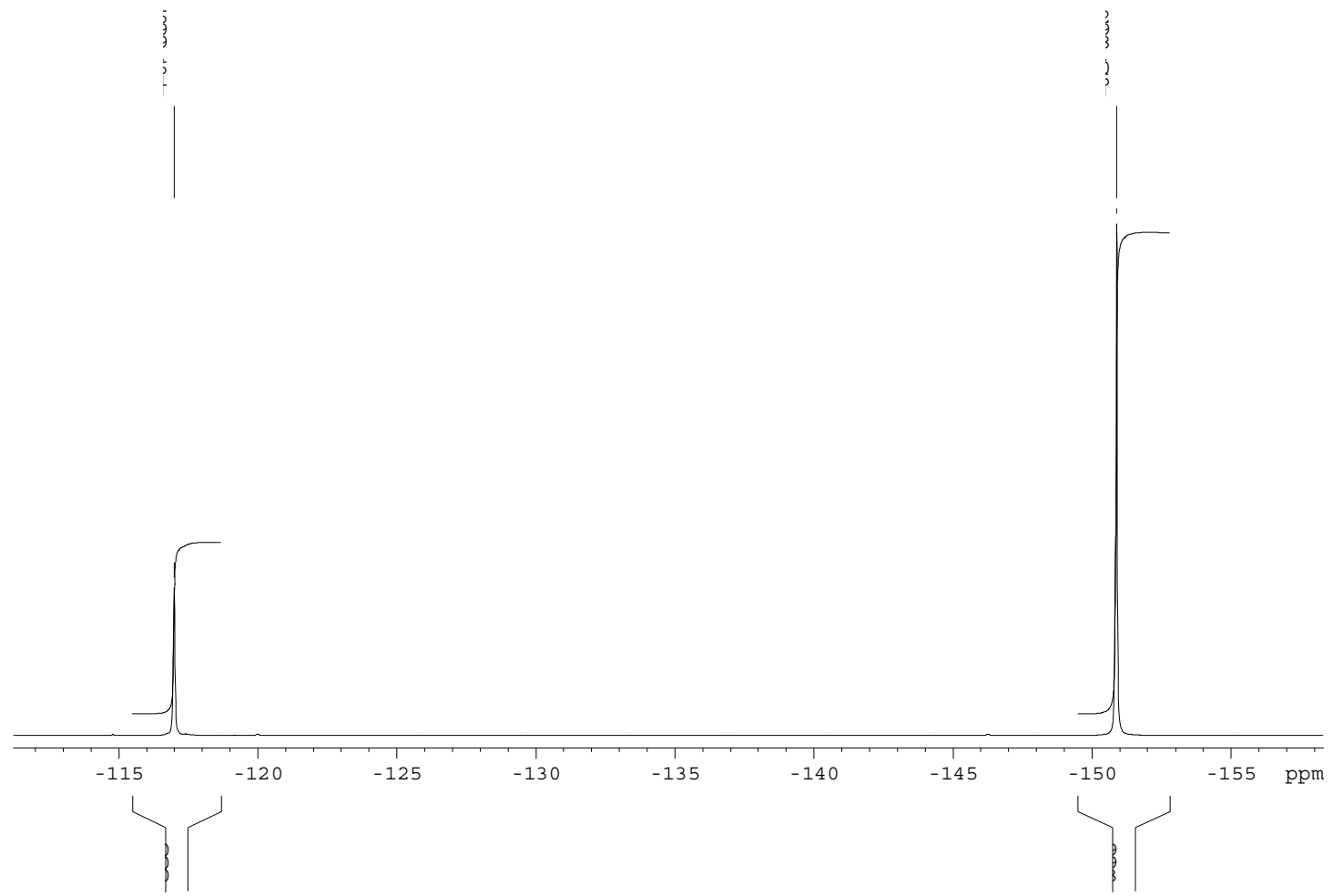




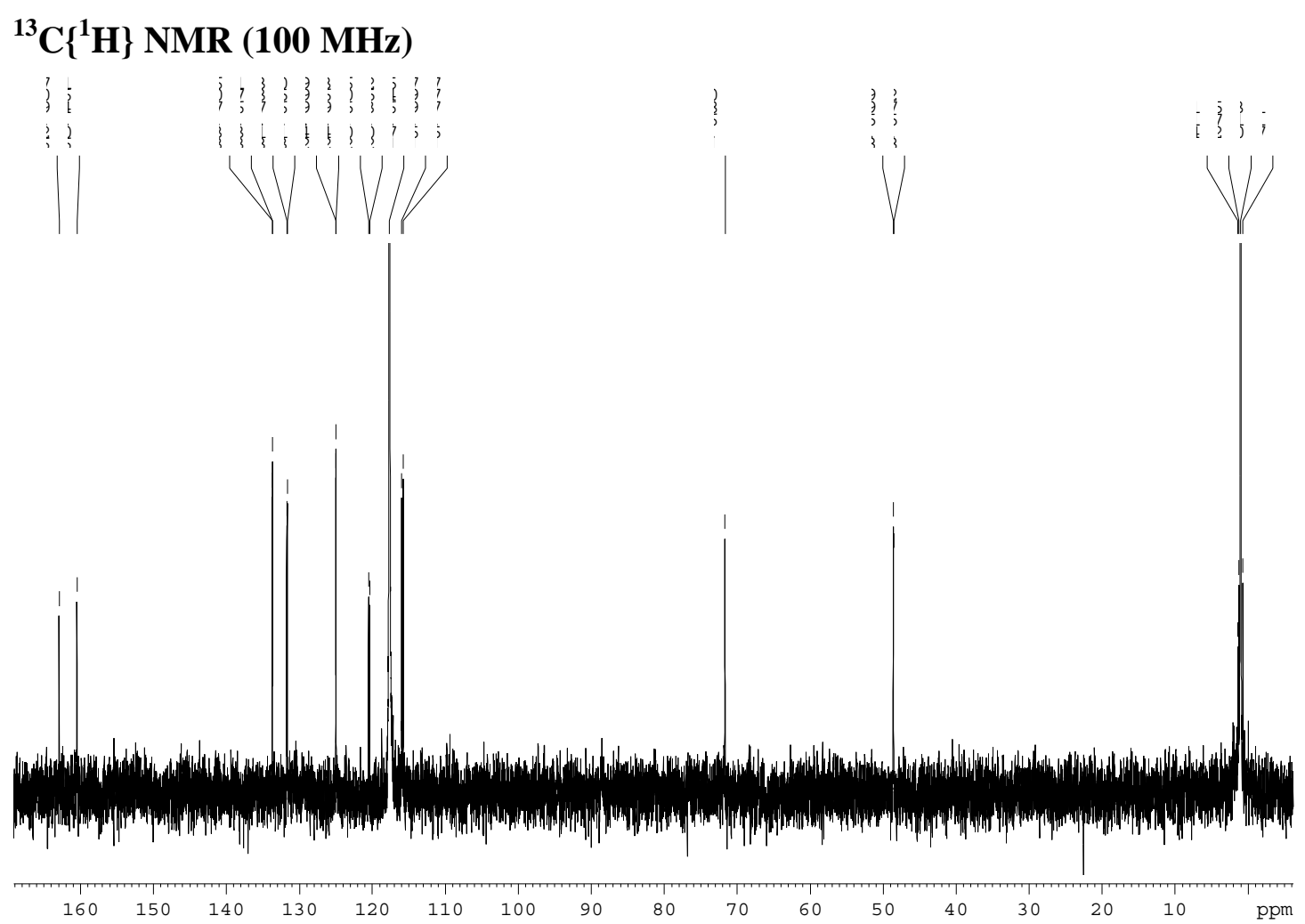

\section{${ }^{13}$ C NMR (100 MHz)}
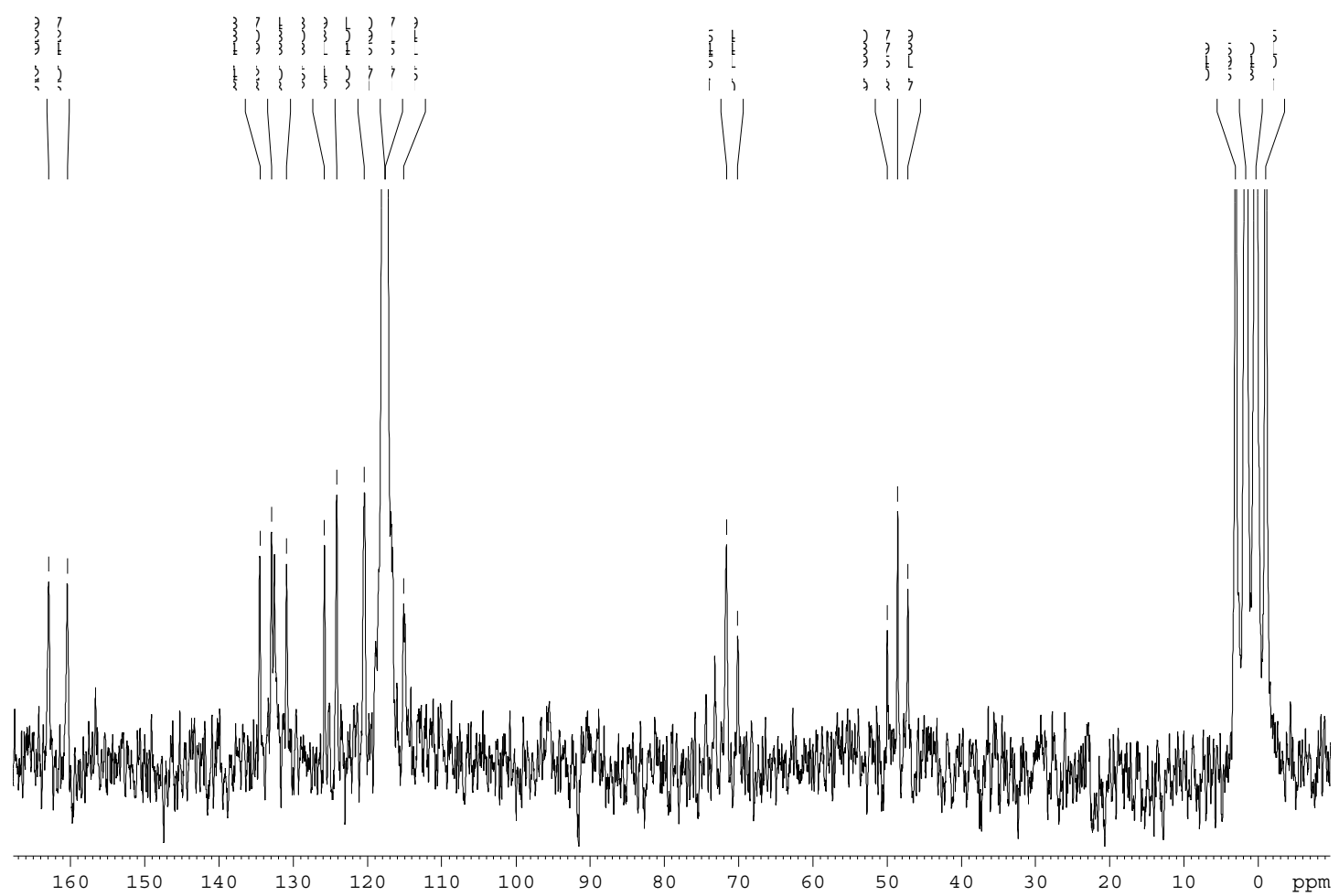
Comparisons of the ${ }^{13} \mathrm{C} \mathrm{CF}$ doublets in the zinc and nickel complex:

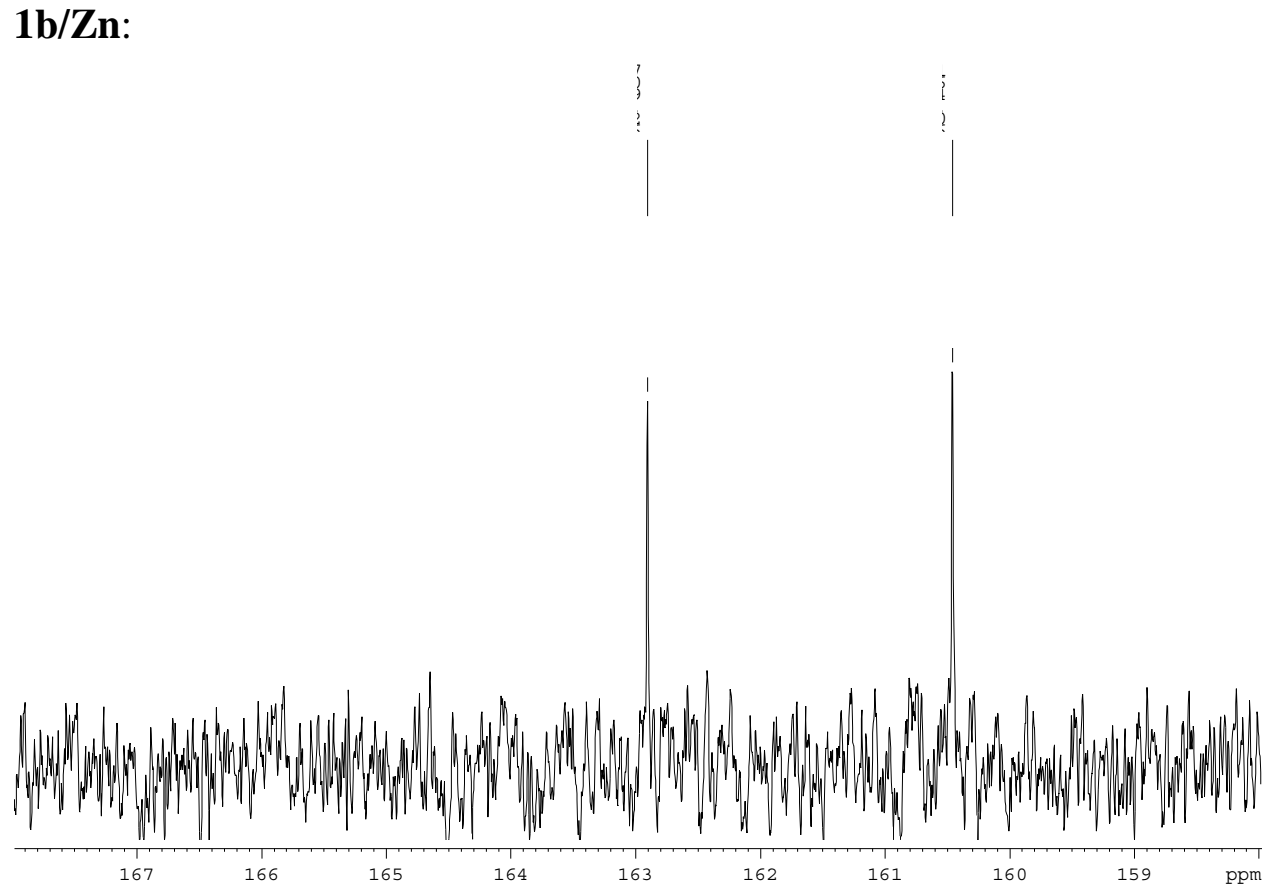

1b:
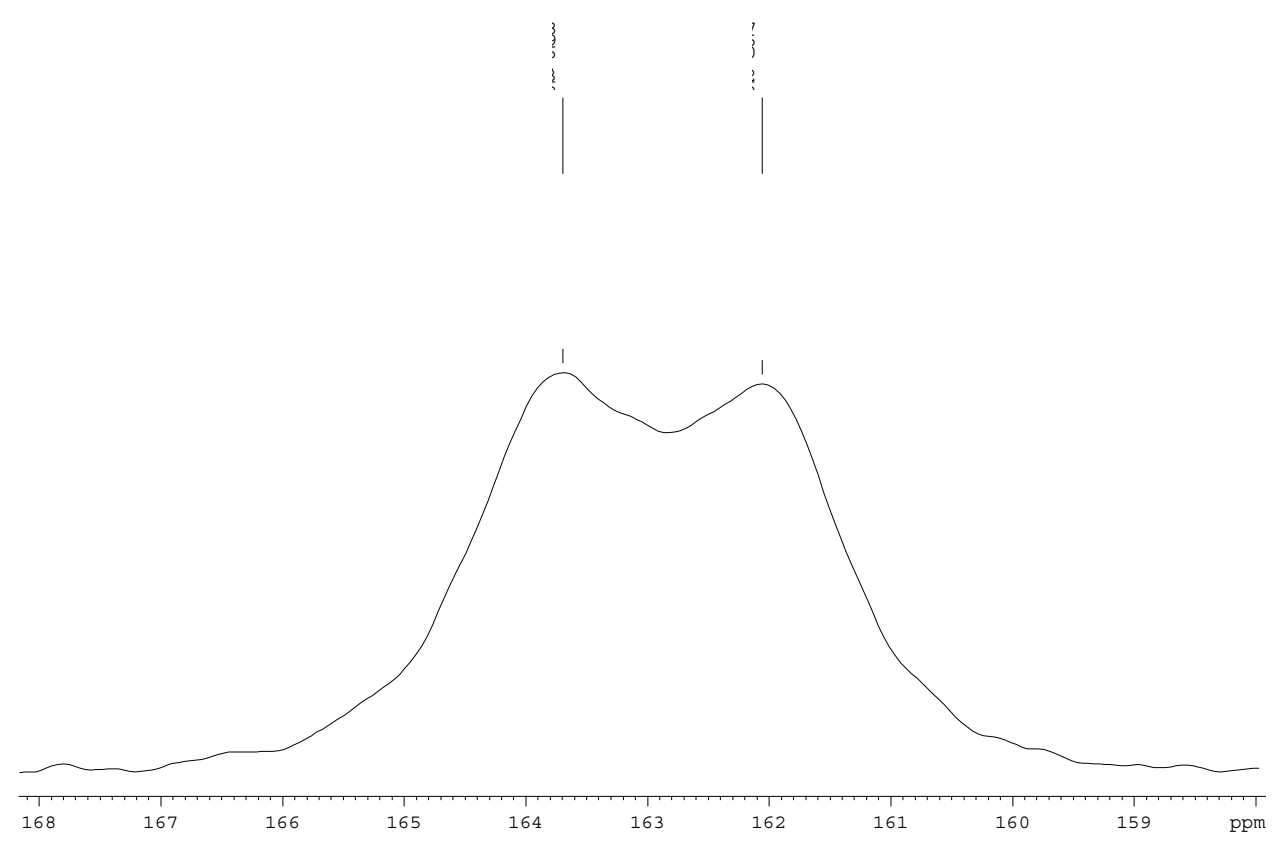
Fit for the broadened C-F doublet:

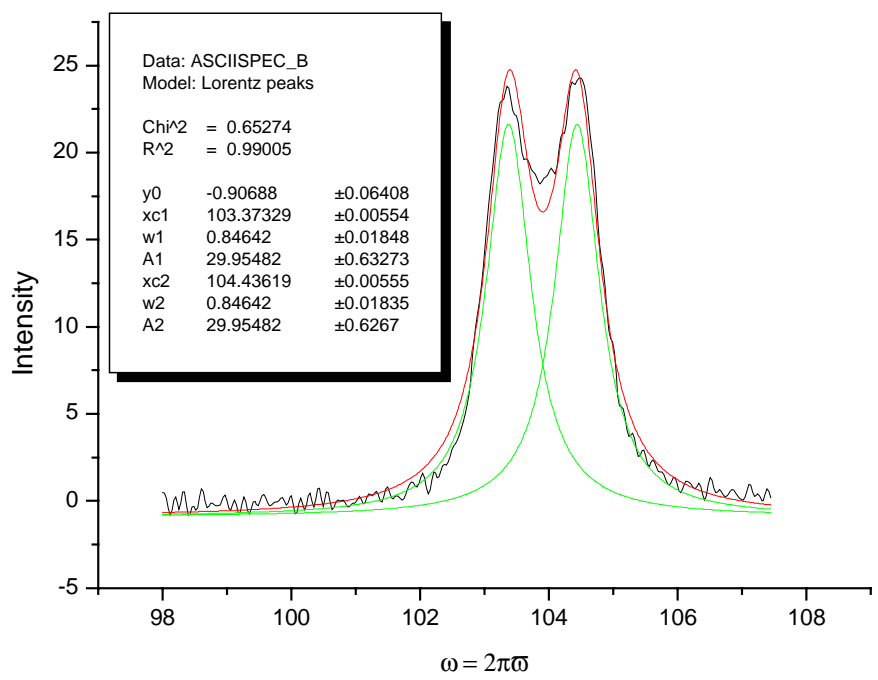

Fit as a Lorentz-shaped doublet is poor. Apparent width is $135(3) \mathrm{Hz}$ with an apparent $\mathrm{J}=169(2) \mathrm{Hz}$.

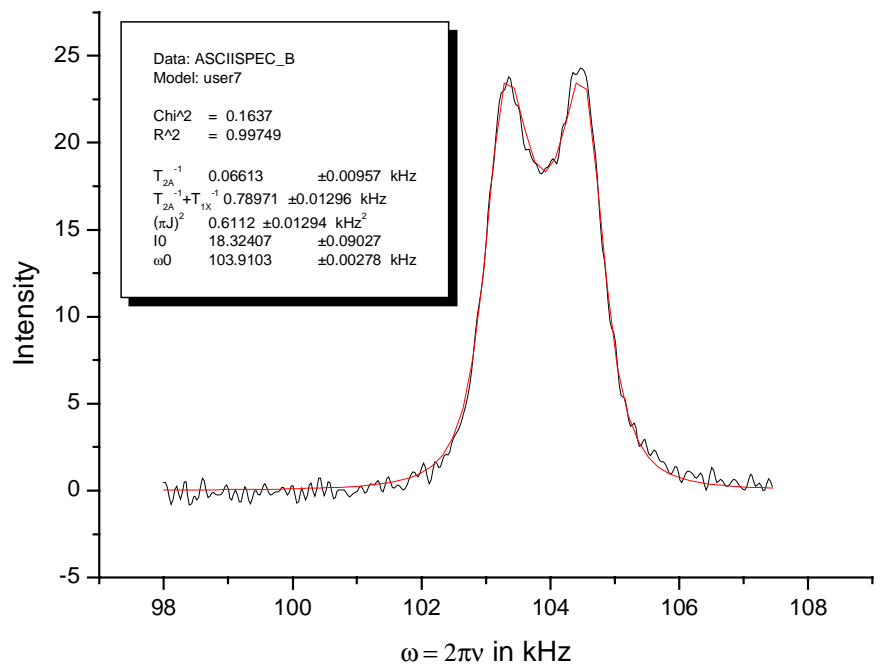

The fit is much better and gives a more reasonable width $\mathrm{T}_{2 \mathrm{~A}}{ }^{-1} / \pi=21(3) \mathrm{Hz}$,

$\mathrm{T}_{1 \mathrm{X}}{ }^{-1}=724(16) \mathrm{Hz}$ and $\mathrm{J}=249(3) \mathrm{Hz}$. These values agree well with the (decoupled) width for the ortho $\mathrm{C}-\mathrm{H}$ (similar distance) of $29 \mathrm{~Hz}$, the inversion recovery $\mathrm{T}_{1 \mathrm{X}}{ }^{-1}=708(1) \mathrm{Hz}$ and $\mathrm{J}=248.9 \mathrm{~Hz}$ in the zinc analog. 
Coupled ${ }^{13} \mathrm{C}$ NMR spectrum for the ortho and para-CH signals in $\mathbf{1 b}$ (compare to the same region for 1a on page S9) with a 10-parameter fit using equation eqS1 for two peaks.

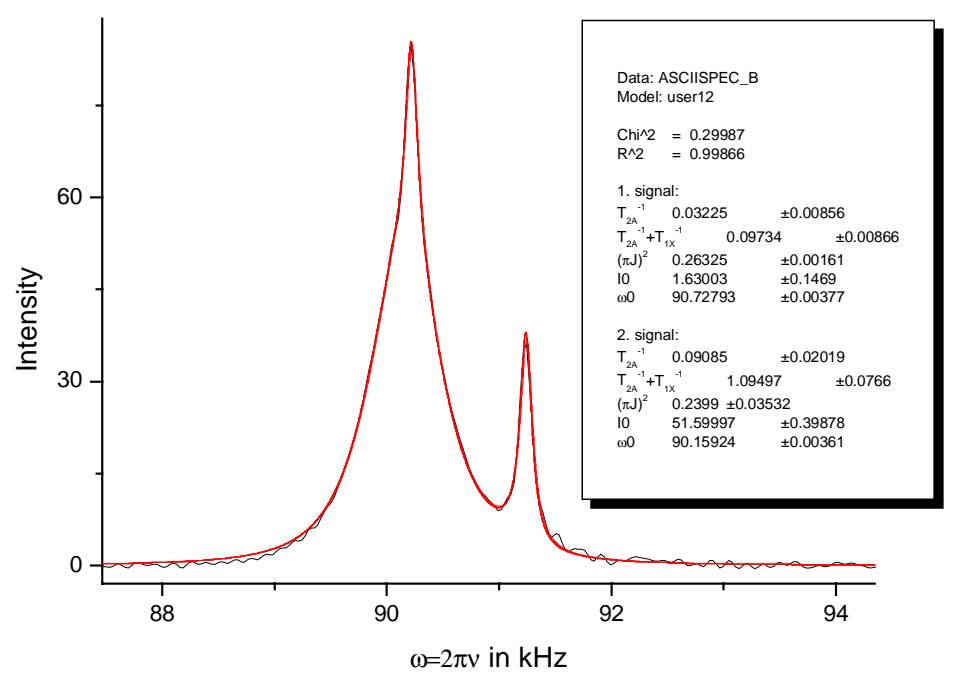

Fit for the $\mathrm{o}$ and $\mathrm{p}-\mathrm{CH}$ :

p-CH: $\delta=143.5-1.6=141.9 \mathrm{ppm}, \mathrm{W}=10(3) \mathrm{Hz}, \mathrm{J}=163(7) \mathrm{Hz}, \mathrm{T}_{1 \mathrm{X}}{ }^{-1}=65(12) \mathrm{Hz}$ o-CH: $\delta=142.6-1.6=141.0 \mathrm{ppm}, \mathrm{W}=29(6) \mathrm{Hz}, \mathrm{J}=156(12) \mathrm{Hz}, \mathrm{T}_{1 \mathrm{X}}{ }^{-1}=1004(79) \mathrm{Hz}$ 


\section{Derivation of equations}

Equations are derived for the Intensity versus $\mathrm{x}=\omega-\omega_{0}$ based on ref $3, \mathrm{~J}$ in $\mathrm{Hz}$ is used (rather than in radian). The real part of the complex expression obtained by the matrix product $\mathbf{W A}^{-1} \mathbf{1}$ has to be derived. As many terms are involved, the procedure is done in many small steps to aid understanding. This leads to full expressions for the line shape for the doublet $(2 \times 2$ matrix), triplet ( $3 \times 3)$ and quartet $(4 \times 4)$ case as eqS1 (page S17), eqS3 (S23) and eqS4 (S30), respectively.

More user friendly approximate solutions for coalesced signals are derived by Taylor expansion around $\mathrm{x}=0$. This over-estimates the apparent line width but gives the appropriate functional forms. This form is corrected empirically, by a best fit procedure as described and validated in detail on page S34.

\section{Doublet Case:}

$$
\begin{aligned}
& A=\left[\begin{array}{cc}
i x+\pi J i-T_{2 A}^{-1}-\frac{1}{2} T_{1 X}^{-1} & \frac{1}{2} T_{1 X}^{-1} \\
\frac{1}{2} T_{1 X}^{-1} & i x-\pi J i-T_{2 A}^{-1}-\frac{1}{2} T_{1 X}^{-1}
\end{array}\right] \\
& x=2 \pi\left(v-v_{0}\right) \\
& |A|=\left(i x-T_{2 A}^{-1}-\frac{1}{2} T_{1 X}^{-1}\right)^{2}+\pi^{2} J^{2}-\frac{1}{4} T_{1 X}^{-2} \\
& A^{-1}=\left[\begin{array}{cc}
i x-\pi J i-T_{2 A}^{-1}-\frac{1}{2} T_{1 X}^{-1} & -\frac{1}{2} T_{1 X}^{-1} \\
-\frac{1}{2} T_{1 X}^{-1} & i x+\pi J i-T_{2 A}^{-1}-\frac{1}{2} T_{1 X}^{-1}
\end{array}\right] / A \mid
\end{aligned}
$$$$
I(x)=\operatorname{Re}\left\{W \bullet A^{-1} \bullet 1\right\}
$$$$
W \bullet A^{-1} \bullet 1=\left(\begin{array}{l}
1 \\
1
\end{array}\right) \bullet A^{-1} \bullet\left(\begin{array}{l}
1 \\
1
\end{array}\right)=2 \frac{i x-T_{2 A}^{-1}-T_{1 X}^{-1}}{\left(i x-T_{2 A}^{-1}-\frac{1}{2} T_{1 X}^{-1}\right)^{2}+\pi^{2} J^{2}-\frac{1}{4} T_{1 X}^{-2}}=
$$$$
=2 \frac{i x-T_{2 A}^{-1}-T_{1 X}^{-1}}{-x^{2}-i x\left(2 T_{2 A}^{-1}+T_{1 X}^{-1}\right)+T_{2 A}^{-2}+T_{2 A}^{-1} T_{1 X}^{-1}+\pi^{2} J^{2}}=
$$$$
=2 \frac{\left(i x-T_{2 A}^{-1}-T_{1 X}^{-1}\right)\left(-x^{2}+i x\left(2 T_{2 A}^{-1}+T_{1 X}^{-1}\right)+T_{2 A}^{-2}+T_{2 A}^{-1} T_{1 X}^{-1}+\pi^{2} J^{2}\right)}{\left(-x^{2}-i x\left(2 T_{2 A}^{-1}+T_{1 X}^{-1}\right)+T_{2 A}^{-2}+T_{2 A}^{-1} T_{1 X}^{-1}+\pi^{2} J^{2}\right)\left(-x^{2}+i x\left(2 T_{2 A}^{-1}+T_{1 X}^{-1}\right)+T_{2 A}^{-2}+T_{2 A}^{-1} T_{1 X}^{-1}+\pi^{2} J^{2}\right)}=
$$$$
=2 \frac{i \operatorname{Im}+\left(T_{2 A}^{-1}+T_{1 X}^{-1}\right)\left(x^{2}-T_{2 A}^{-2}-T_{2 A}^{-1} T_{1 X}^{-1}-\pi^{2} J^{2}\right)-\left(2 T_{2 A}^{-1}+T_{1 X}^{-1}\right) x^{2}}{\left(-x^{2}+T_{2 A}^{-2}+T_{2 A}^{-1} T_{1 X}^{-1}+\pi^{2} J^{2}\right)^{2}+x^{2}\left(2 T_{2 A}^{-1}+T_{1 X}^{-1}\right)^{2}}=
$$$$
=2 \frac{i \operatorname{Im}-\left(T_{2 A}^{-1}+T_{1 X}^{-1}\right)\left(T_{2 A}^{-2}+T_{2 A}^{-1} T_{1 X}^{-1}+\pi^{2} J^{2}\right)-T_{2 A}^{-1} x^{2}}{x^{4}+x^{2}\left(\left(2 T_{2 A}^{-1}+T_{1 X}^{-1}\right)^{2}-2 T_{2 A}^{-2}-2 T_{2 A}^{-1} T_{1 X}^{-1}-2 \pi^{2} J^{2}\right)+\left(T_{2 A}^{-2}+T_{2 A}^{-1} T_{1 X}^{-1}+\pi^{2} J^{2}\right)^{2}}=
$$$$
=2 \frac{i \operatorname{Im}-\left(T_{2 A}^{-1}+T_{1 X}^{-1}\right)\left(T_{2 A}^{-2}+T_{2 A}^{-1} T_{1 X}^{-1}+\pi^{2} J^{2}\right)-T_{2 A}^{-1} x^{2}}{x^{4}+x^{2}\left(2 T_{2 A}^{-2}+2 T_{2 A}^{-1} T_{1 X}^{-1}+T_{1 X}^{-2}-2 \pi^{2} J^{2}\right)+\left(T_{2 A}^{-2}+T_{2 A}^{-1} T_{1 X}^{-1}+\pi^{2} J^{2}\right)^{2}}
$$

With $\mathrm{I}(\mathrm{x}=0)=\mathrm{I}_{0}=\frac{-2\left(T_{2 A}^{-1}+T_{1 X}^{-1}\right)}{\left(T_{2 A}^{-2}+T_{2 A}^{-1} T_{1 X}^{-1}+\pi^{2} J^{2}\right)}$ 
$I(x)=I_{0} \frac{\left(T_{2 A}^{-2}+T_{2 A}^{-1} T_{1 X}^{-1}+\pi^{2} J^{2}\right) \frac{T_{2 A}^{-1}}{T_{2 A}^{-1}+T_{1 X}^{-1}} x^{2}+\left(T_{2 A}^{-2}+T_{2 A}^{-1} T_{1 X}^{-1}+\pi^{2} J^{2}\right)^{2}}{x^{4}+x^{2}\left(2 T_{2 A}^{-2}+2 T_{2 A}^{-1} T_{1 X}^{-1}+T_{1 X}^{-2}-2 \pi^{2} J^{2}\right)+\left(T_{2 A}^{-2}+T_{2 A}^{-1} T_{1 X}^{-1}+\pi^{2} J^{2}\right)^{2}}$

with

$x=\omega-\omega_{0}$

$P 1=T_{2 A}^{-1} ; P 2=T_{2 A}^{-1}+T_{1 X}^{-1} ; P 3=\pi^{2} J^{2} ; P 4=I_{0} ; P 5=\omega_{0}$

$I(x)=P 4 \frac{(P 1 P 2+P 3) \frac{P 1}{P 2}(\omega-P 5)^{2}+(P 1 P 2+P 3)^{2}}{(\omega-P 5)^{4}+(\omega-P 5)^{2}\left(P 1^{2}+P 2^{2}-2 P 3\right)+(P 1 P 2+P 3)^{2}}(e q S 1)$

The latter equation (eqS1) is used as user defined equation for 5 parameter fitting in ORIGIN (or 10 parameter fitting for two peaks) 
Conditions for coalescence to a single peak:

$$
\begin{aligned}
& I(x)=I_{0} \frac{\left(T_{2 A}^{-2}+T_{2 A}^{-1} T_{1 X}^{-1}+\pi^{2} J^{2}\right)_{\frac{T_{2 A}^{-A}}{T_{2}^{-1}}+T_{1 X}^{-1}} x^{2}+\left(T_{2 A}^{-2}+T_{2 A}^{-1} T_{1 X}^{-1}+\pi^{2} J^{2}\right)^{2}}{x^{4}+x^{2}\left(2 T_{2 A}^{-2}+2 T_{2 A}^{-1} T_{1 X}^{-1}+T_{1 X}^{-2}-2 \pi^{2} J^{2}\right)+\left(T_{2 A}^{-2}+T_{2 A}^{-1} T_{1 X}^{-1}+\pi^{2} J^{2}\right)^{2}}=I_{0} \frac{A x^{2}+B}{x^{4}+C x^{2}+B} \\
& I^{\prime}(x)=I_{0} \frac{2 A x\left(x^{4}+C x^{2}+B\right)-\left(4 x^{3}+2 C x\right)\left(A x^{2}+B\right)}{\left(x^{4}+C x^{2}+B\right)^{2}}= \\
& =I_{0} \frac{2 A x^{5}+2 A C x^{3}+2 A B x-4 A x^{5}-2 A C x^{3}-4 B x^{3}-2 B C x}{\left(x^{4}+C x^{2}+B\right)^{2}}= \\
& =I_{0} \frac{-2 A x^{5}-4 B x^{3}+2(A-C) B x}{\left(x^{4}+C x^{2}+B\right)^{2}}
\end{aligned}
$$

coalescence:

$$
\begin{aligned}
& I^{\prime \prime}(x=0)=I_{0} \frac{2(A-C) B^{2}}{B^{3}}=0 \Rightarrow A=C \\
& \left(T_{2 A}^{-2}+T_{2 A}^{-1} T_{1 X}^{-1}+\pi^{2} J^{2}\right) \frac{T_{2 A}^{-1}}{T_{2 A}^{-1}+T_{1 X}^{-1}}=2 T_{2 A}^{-2}+2 T_{2 A}^{-1} T_{1 X}^{-1}+T_{1 X}^{-2}-2 \pi^{2} J^{2} \\
& T_{2 A}^{-3}+T_{2 A}^{-2} T_{1 X}^{-1}+T_{2 A}^{-1} \pi^{2} J^{2}=2 T_{2 A}^{-3}+2 T_{2 A}^{-2} T_{1 X}^{-1}+T_{2 A}^{-1} T_{1 X}^{-2}-2 T_{2 A}^{-1} \pi^{2} J^{2}+2 T_{1 X}^{-1} T_{2 A}^{-2}+2 T_{2 A}^{-1} T_{1 X}^{-2}+T_{1 X}^{-3}-2 T_{1 X}^{-1} \pi^{2} J^{2} \\
& \left(3 T_{2 A}^{-1}+2 T_{1 X}^{-1}\right) \pi^{2} J^{2}=T_{2 A}^{-3}+3 T_{2 A}^{-2} T_{1 X}^{-1}+3 T_{2 A}^{-1} T_{1 X}^{-2}+T_{1 X}^{-3}=\left(T_{1 X}^{-1}+T_{2 A}^{-1}\right)^{3} \\
& \pi^{2} J^{2}=\frac{\left(T_{1 X}^{-1}+T_{2 A}^{-1}\right)^{3}}{3 T_{2 A}^{-1}+2 T_{1 X}^{-1}}(\text { eq. } 3)
\end{aligned}
$$


Approximate solution for the maxima at $\mathrm{x}=+/-\pi \Delta$ ( $\Delta$ apparent $\mathrm{J})$ before coalescence:

$$
\begin{aligned}
& I^{\prime}(\pi \Delta)=I_{0} \frac{-2 A(\pi \Delta)^{5}-4 B(\pi \Delta)^{3}+2(A-C) B(\pi \Delta)}{\left((\pi \Delta)^{4}+C(\pi \Delta)^{2}+B\right)^{2}}=0 \\
& \Rightarrow-2 A(\pi \Delta)^{4}-4 B(\pi \Delta)^{2}+2(A-C) B=0 \\
& (\pi \Delta)^{4}+2 \frac{B}{A}(\pi \Delta)^{2}-(A-C) \frac{B}{A}=0 \\
& (\pi \Delta)^{2}=-\frac{B}{A}+\sqrt{\left(\frac{B}{A}\right)^{2}+B+C \frac{B}{A}}=-\left(T_{2 A}^{-2}+2 T_{2 A}^{-1} T_{1 X}^{-1}+T_{1 X}^{-2}+\pi^{2} J^{2}+\pi^{2} J^{2} \frac{T_{1 X}^{-1}}{T_{2 A}^{-1}}\right) \\
& +\sqrt{\begin{array}{l}
\left(T_{2 A}^{-2}+2 T_{2 A}^{-1} T_{1 X}^{-1}+T_{1 X}^{-2}+\pi^{2} J^{2}+\pi^{2} J^{2} \frac{T_{1 X}^{-1}}{T_{2 A}^{-1}}\right)^{2}+\left(T_{2 A}^{-2}+T_{2 A}^{-1} T_{1 X}^{-1}+\pi^{2} J^{2}\right)^{2} \\
-\left(2 T_{2 A}^{-2}+2 T_{2 A}^{-1} T_{1 X}^{-1}+T_{1 X}^{-2}-2 \pi^{2} J^{2}\right)\left(T_{2 A}^{-2}+2 T_{2 A}^{-1} T_{1 X}^{-1}+T_{1 X}^{-2}+\pi^{2} J^{2}+\pi^{2} J^{2} \frac{T_{1 X}^{-1}}{T_{2 A}^{-1}}\right)
\end{array}}= \\
& =-\left(T_{2 A}^{-1}+T_{1 X}^{-1}\right)^{2}-\left(1+\frac{T_{1 X}^{-1}}{T_{2 A}^{-1}}\right) \pi^{2} J^{2} \\
& +\sqrt{\begin{array}{l}
\left(T_{2 A}^{-1}+T_{1 X}^{-1}\right)^{4}+2\left(T_{2 A}^{-1}+T_{1 X}^{-1}\right)^{2}\left(1+\frac{T_{1 X}^{-1}}{T_{2 A}^{-1}}\right) \pi^{2} J^{2}+\left(1+\frac{T_{1 X}^{-1}}{T_{2 A}^{-1}}\right)^{2} \pi^{4} J^{4}+T_{2 A}^{-2}\left(T_{2 A}^{-1}+T_{1 X}^{-1}\right)^{2} \\
+\left(T_{2 A}^{-1}+T_{1 X}^{-1}\right) \pi^{2} J^{2}+\pi^{4} J^{4}-T_{2 A}^{-2}\left(T_{2 A}^{-1}+T_{1 X}^{-1}\right)^{2}-T_{2 A}^{-2}\left(1+\frac{T_{1 X}^{-1}}{T_{2 A}^{-1}}\right) \pi^{2} J^{2}-\left(T_{2 A}^{-1}+T_{1 X}^{-1}\right)^{4}= \\
-\left(T_{2 A}^{-1}+T_{1 X}^{-1}\right)^{2}\left(1+\frac{T_{1 X}^{-1}}{T_{2 A}^{-1}}\right) \pi^{2} J^{2}+2\left(T_{2 A}^{-1}+T_{1 X}^{-1}\right)^{2} \pi^{2} J^{2}+2\left(1+\frac{T_{1 X}^{-1}}{T_{2 A}^{-1}}\right) \pi^{4} J^{4}
\end{array}} \\
& =-\left(T_{2 A}^{-1}+T_{1 X}^{-1}\right)^{2}-\left(1+\frac{T_{1 X}^{-1}}{T_{2 A}^{-1}}\right) \pi^{2} J^{2}
\end{aligned}
$$

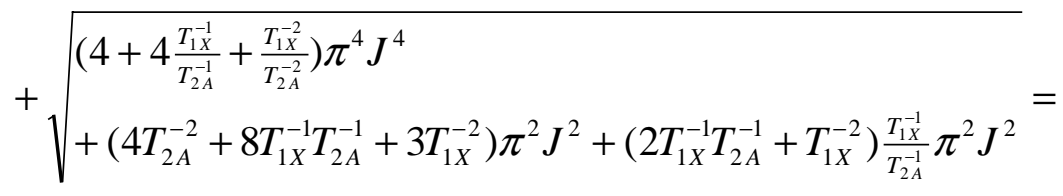

$$
\begin{aligned}
& =-\left(T_{2 A}^{-1}+T_{1 X}^{-1}\right)^{2}-\left(1+\frac{T_{1 X}^{-1}}{T_{2 A}^{-1}}\right) \pi^{2} J^{2} \\
& +\sqrt{\left(4 T_{2 A}^{-2}+8 T_{1 X}^{-1} T_{2 A}^{-1}+5 T_{1 X}^{-2}+\frac{T_{1 X}^{-3}}{T_{2 A}^{-1}}\right) \pi^{2} J^{2}+\left(2+\frac{T_{1 X}^{-1}}{T_{2 A}^{-1}}\right)^{2} \pi^{4} J^{4}}= \\
& =T_{2 A}\left[-T_{2 A}^{-1}\left(T_{2 A}^{-1}+T_{1 X}^{-1}\right)^{2}-\left(T_{2 A}^{-1}+T_{1 X}^{-1}\right) \pi^{2} J^{2}+\right. \\
& \left.\sqrt{\left(4 T_{2 A}^{-4}+8 T_{1 X}^{-1} T_{2 A}^{-3}+5 T_{1 X}^{-2} T_{2 A}^{-2}+T_{1 X}^{-3} T_{2 A}^{-1}\right) \pi^{2} J^{2}+\left(2 T_{2 A}^{-1}+T_{1 X}^{-1}\right)^{2} \pi^{4} J^{4}}\right]
\end{aligned}
$$

Expansion in $\mathrm{T}_{2 \mathrm{~A}}{ }^{-1}\left(<<\mathrm{T}_{1 \mathrm{X}}{ }^{-1}, \pi \mathrm{J}\right)$ to second order:

$$
\begin{aligned}
& (\pi \Delta)^{2}=T_{2 A}\left[-T_{1 X}^{-1} \pi^{2} J^{2}+\sqrt{T_{1 X}^{-2} \pi^{4} J^{4}}\right] \\
& -T_{1 X}^{-2}-\pi^{2} J^{2}+\left[T_{1 X}^{-3} \pi^{2} J^{2}+4 T_{1 X}^{-1} \pi^{4} J^{4}\right] / 2 \sqrt{T_{1 X}^{-2} \pi^{4} J^{4}} \\
& -\frac{1}{2} T_{2 A}^{-1} T_{1 X}^{-1}-\frac{T_{2 A}^{-1} T_{1 X}^{-3}}{8 \pi^{2} J^{2}}=-T_{1 X}^{-2}-\pi^{2} J^{2}+\frac{1}{2} T_{1 X}^{-2}+2 \pi^{2} J^{2}-\frac{1}{2} T_{2 A}^{-1} T_{1 X}^{-1}-\frac{T_{2 A}^{-1} T_{1 X}^{-3}}{8 \pi^{2} J^{2}}= \\
& =\pi^{2} J^{2}-\frac{1}{2} T_{1 X}^{-2}-\frac{1}{2} T_{2 A}^{-1} T_{1 X}^{-1}-\frac{T_{2 A}^{-1} T_{1 X}^{-3}}{8 \pi^{2} J^{2}} \\
& \approx \pi^{2} J^{2}-\frac{1}{2} T_{1 X}^{-2}
\end{aligned}
$$


Approximate solutions for the line widths of the coalesced peak:

- for singlets: Taylor expansion of $1 /\left\{\mathrm{WA}^{-1} 1\right\}$ in (ix) gives:

$$
\begin{aligned}
& f(i x)=2 /\left(W \bullet A^{-1} \bullet 1\right)=\frac{\left(i x-T_{2 A}^{-1}-\frac{1}{2} T_{1 X}^{-1}\right)^{2}+\pi^{2} J^{2}-\frac{1}{4} T_{1 X}^{-2}}{i x-T_{2 A}^{-1}-T_{1 X}^{-1}} \\
& f(0)=\frac{\left(T_{2 A}^{-1}+\frac{1}{2} T_{1 X}^{-1}\right)^{2}+\pi^{2} J^{2}-\frac{1}{4} T_{1 X}^{-2}}{T_{2 A}^{-1}+T_{1 X}^{-1}}=T_{2 A}^{-1}+\frac{\pi^{2} J^{2}}{T_{2 A}^{-1}+T_{1 X}^{-1}} \\
& f^{\prime}(i x)=\frac{2\left(i x-T_{2 A}^{-1}-T_{1 X}^{-1}\right)\left(i x-T_{2 A}^{-1}-\frac{1}{2} T_{1 X}^{-1}\right)-\left(i x-T_{2 A}^{-1}-\frac{1}{2} T_{1 X}^{-1}\right)^{2}-\pi^{2} J^{2}+\frac{1}{4} T_{1 X}^{-2}}{\left(i x-T_{2 A}^{-1}-T_{1 X}^{-1}\right)^{2}}= \\
& =\frac{2(i x)^{2}-2\left(T_{2 A}^{-1}+T_{1 X}^{-1}\right)(i x)+2\left(T_{2 A}^{-1}+T_{1 X}^{-1}\right)\left(T_{2 A}^{-1}+\frac{1}{2} T_{1 X}^{-1}\right)-\left(T_{2 A}^{-1}+\frac{1}{2} T_{1 X}^{-1}\right)^{2}-\pi^{2} J^{2}+\frac{1}{4} T_{1 X}^{-2}}{\left(i x-T_{2 A}^{-1}-T_{1 X}^{-1}\right)^{2}}= \\
& =\frac{2(i x)^{2}-2\left(T_{2 A}^{-1}+T_{1 X}^{-1}\right)(i x)+\left(T_{2 A}^{-1}+T_{1 X}^{-1}\right)^{2}-\pi^{2} J^{2}}{\left(i x-T_{2 A}^{-1}-T_{1 X}^{-1}\right)^{2}} \\
& \Rightarrow f^{\prime}(0)=\frac{\left(T_{2 A}^{-1}+T_{1 X}^{-1}\right)^{2}-\pi^{2} J^{2}}{\left(T_{2 A}^{-1}+T_{1 X}^{-1}\right)^{2}} \\
& \Rightarrow
\end{aligned}
$$

$$
\begin{aligned}
& f(i x) \approx T_{2 A}^{-1}+\frac{\pi^{2} J^{2}}{T_{2 A}^{-1}+T_{1 X}^{-1}}+\frac{\left(T_{2 A}^{-1}+T_{1 X}^{-1}\right)^{2}-\pi^{2} J^{2}}{\left(T_{2 A}^{-1}+T_{1 X}^{-1}\right)^{2}}(i x)= \\
& =\frac{\left(T_{2 A}^{-1}+T_{1 X}^{-1}\right)^{2}-\pi^{2} J^{2}}{\left(T_{2 A}^{-1}+T_{1 X}^{-1}\right)^{2}}(\underbrace{\frac{\left(T_{2 A}^{-1}+T_{1 X}^{-1}\right)^{2} T_{2 A}^{-1}+\left(T_{2 A}^{-1}+T_{1 X}^{-1}\right) \pi^{2} J^{2}}{\left(T_{2 A}^{-1}+T_{1 X}^{-1}\right)^{2}-\pi^{2} J^{2}}+(i x)}_{\pi W})
\end{aligned}
$$

Taylor expansion in $\pi^{2} \mathrm{~J}^{2}$ :

$\Rightarrow \pi W \approx T_{2 A}^{-1}+\frac{T_{1 X}^{-1}+2 T_{2 A}^{-1}}{\left(T_{2 A}^{-1}+T_{1 X}^{-1}\right)^{2}} \pi^{2} J^{2}$ (eq.S2)

This approximation constructs an approximate Lorentz peak around $\mathrm{x}=0$. The true line shape is "flattened" at this point, thus (eq. S2) over-estimates the widths compared to the least-square Lorentz fit. 
Taylor expansion around $\mathrm{y}=1 / \mathrm{x}=0$ (infinite $\mathrm{x}$ ):

$$
\begin{aligned}
& I(x)=C \frac{i x-T_{2 A}^{-1}-T_{1 X}^{-1}}{-x^{2}-i x\left(2 T_{2 A}^{-1}+T_{1 X}^{-1}\right)+T_{2 A}^{-2}+T_{2 A}^{-1} T_{1 X}^{-1}+\pi^{2} J^{2}}= \\
& =C \frac{i y-\left(T_{2 A}^{-1}+T_{1 X}^{-1}\right) y^{2}}{-1-i y\left(2 T_{2 A}^{-1}+T_{1 X}^{-1}\right)+\ldots} \\
& I(y=0)=0 \\
& I^{\prime}(y)=C \frac{\left(-1-i y\left(2 T_{2 A}^{-1}+T_{1 X}^{-1}\right)\right)\left(i-2\left(T_{2 A}^{-1}+T_{1 X}^{-1}\right) y\right)+i y\left(2 T_{2 A}^{-1}+T_{1 X}^{-1}\right)\left(i-\left(T_{2 A}^{-1}+T_{1 X}^{-1}\right) y\right)+\ldots}{\left(-1-i y\left(2 T_{2 A}^{-1}+T_{1 X}^{-1}\right)+\ldots\right)^{2}}= \\
& =C \frac{-i+y\left(2 T_{2 A}^{-1}+2 T_{1 X}^{-1}\right)+\ldots}{1+2 i y\left(2 T_{2 A}^{-1}+T_{1 X}^{-1}\right)+\ldots} \quad\left(1+2 i y\left(2 T_{2 A}^{-1}+T_{1 X}^{-1}\right)+\ldots\right)^{2} \\
& I^{\prime}(y=0)=-C i \\
& I^{\prime \prime}(y)=C \frac{\left(1+2 i y\left(2 T_{2 A}^{-1}+T_{1 X}^{-1}\right)\right)\left(2 T_{2 A}^{-1}+2 T_{X}^{-1}\right)-2 i\left(2 T_{2 A}^{-1}+T_{1 X}^{-1}\right)\left(-i+y\left(2 T_{2 A}^{-1}+2 T_{1 X}^{-1}\right)\right)+\ldots}{(1}=-2 C T_{2 A}^{-1} \\
& I^{\prime \prime}(y=0)=C \frac{2 T_{2 A}^{-1}+2 T_{1 X}^{-1}-2\left(2 T_{2 A}^{-1}+T_{1 X}^{-1}\right)}{I^{2}}=-C\left(i y+T_{2 A}^{-1} y^{2}\right) \\
& I^{\prime}(y) \approx-C \\
& \text { and } \\
& \mid \operatorname{Re}(I(y)) \approx C T_{2 A}^{-1} y^{2}=C \frac{T_{2 A}^{-1}}{x^{2}}
\end{aligned}
$$

Expansion of a Lorentz function with width $\mathrm{W}$ around $\mathrm{y}=1 / \mathrm{x}=0$ :

$$
\begin{aligned}
& I(x)=\frac{C}{i x+\pi W}=C \frac{y}{i+\pi W y} \\
& I(y=0)=0 \\
& I^{\prime}(y)=C \frac{(i+\pi W y) 1-\pi W y}{(i+\pi W y)^{2}}=C \frac{i}{-1+2 \pi W y i+\ldots} \\
& I^{\prime}(y=0)=-C i \\
& I^{\prime \prime}(y)=C \frac{(-1+2 \pi W y i) 0+2 \pi W+\ldots}{(-1+2 \pi W y i+\ldots)^{2}}=C \frac{2 \pi W+\ldots}{1-4 \pi W y i+\ldots} \\
& I^{\prime \prime}(y=0)=2 \pi W C \\
& I(y) \approx-C i y+\pi W C y^{2} \\
& \text { and }
\end{aligned}
$$$$
\operatorname{Re}(I(y)) \approx \pi W C y^{2}=C \frac{\pi W}{x^{2}}
$$

Therefore:

$\pi W \approx T_{2 A}^{-1}$

This approximation under-estimates the width for a least-square Lorentz fit. 
Triplet Case:

$$
\begin{aligned}
& A=\left[\begin{array}{ccc}
i x+2 \pi J i-T_{2 A}^{-1}-T_{1 X}^{-1} & T_{1 X}^{-1} & 0 \\
\frac{1}{2} T_{1 X}^{-1} & i x-T_{2 A}^{-1}-T_{1 X}^{-1} & \frac{1}{2} T_{1 X}^{-1} \\
0 & T_{1 X}^{-1} & i x-2 \pi J i-T_{2 A}^{-1}-T_{1 X}^{-1}
\end{array}\right] \\
& x=2 \pi\left(v-v_{0}\right) \\
& |A|=\left(i x-T_{2 A}^{-1}-T_{1 X}^{-1}\right)^{3}+4 \pi^{2} J^{2}\left(i x-T_{2 A}^{-1}-T_{1 X}^{-1}\right)-\left(i x-T_{2 A}^{-1}-T_{1 X}^{-1}\right) T_{1 X}^{-2}= \\
& =(i x)^{3}-3\left(T_{2 A}^{-1}+T_{1 X}^{-1}\right)(i x)^{2}+\left(3\left(T_{2 A}^{-1}+T_{1 X}^{-1}\right)^{2}+4 \pi^{2} J^{2}-T_{1 X}^{-2}\right)(i x)-\left(T_{2 A}^{-1}+T_{1 X}^{-1}\right)^{3} \\
& -4 \pi^{2} J^{2}\left(T_{2 A}^{-1}+T_{1 X}^{-1}\right)+T_{2 A}^{-1} T_{1 X}^{-2}-T_{1 X}^{-3}= \\
& =(i x)^{3}-3\left(T_{2 A}^{-1}+T_{1 X}^{-1}\right)(i x)^{2}+\left(3 T_{2 A}^{-2}+6 T_{2 A}^{-1} T_{1 X}^{-1}+2 T_{1 X}^{-2}+4 \pi^{2} J^{2}\right)(i x) \\
& -T_{2 A}^{-3}-3 T_{2 A}^{-2} T_{1 X}^{-1}-2 T_{2 A}^{-1} T_{1 X}^{-2}-4 \pi^{2} J^{2}\left(T_{2 A}^{-1}+T_{1 X}^{-1}\right) \\
& A^{-1}= \\
& =\frac{\left[\begin{array}{ccc}
\left(i x-T_{2 A}^{-1}-T_{1 X}^{-1}\right)^{2} & \multicolumn{1}{c}{} & \multicolumn{1}{c}{T_{1 X}^{-2}} \\
-2 \pi J i\left(i x-T_{2 A}^{-1}-T_{1 X}^{-1}\right)-\frac{1}{2} T_{1 X}^{-2} & -T_{1 X}^{-1}\left(i x-T_{2 A}^{-1}-T_{1 X}^{-1}-2 \pi J i\right) & \multicolumn{1}{c}{{ }^{-1}\left(i x-T_{2 A}^{-1}-T_{1 X}^{-1}+2 \pi J i\right)} \\
-\frac{1}{2} T_{1 X}^{-1}\left(i x-T_{2 A}^{-1}-T_{1 X}^{-1}-2 \pi J i\right) & \left(i x-T_{2 A}^{-1}-T_{1 X}^{-1}\right)^{2}+4 \pi^{2} J^{2} & -\frac{1}{2} T_{1 X}^{-1}\left(i x-T_{1 X}^{-1}\right)^{2} \\
\frac{1}{2} T_{1 X}^{-2} & -T_{1 X}^{-1}\left(i x-T_{2 A}^{-1}-T_{1 X}^{-1}+2 \pi J i\right) & \left(i x-T_{2 A}^{-1}-T_{1 X}^{-1}\right. \\
+2 \pi J i\left(i x-T_{2 A}^{-1}-T_{1 X}^{-1}\right)-\frac{1}{2} T_{1 X}^{-2}
\end{array}\right]}{|A|}
\end{aligned}
$$




$$
\begin{aligned}
& I(x)=\operatorname{Re}\left\{W \bullet A^{-1} \bullet 1\right\} \\
& W \bullet A^{-1} \bullet 1=\left(\begin{array}{l}
1 \\
2 \\
1
\end{array}\right) \bullet A^{-1} \bullet\left(\begin{array}{l}
1 \\
1 \\
1
\end{array}\right)= \\
& =\frac{4\left(i x-T_{2 A}^{-1}-T_{1 X}^{-1}\right)^{2}-4 T_{1 X}^{-1}\left(i x-T_{2 A}^{-1}-T_{1 X}^{-1}\right)+8 \pi^{2} J^{2}}{(i x)^{3}-3\left(T_{2 A}^{-1}+T_{1 X}^{-1}\right)(i x)^{2}+\left(3 T_{2 A}^{-2}+6 T_{2 A}^{-1} T_{1 X}^{-1}+2 T_{1 X}^{-2}+4 \pi^{2} J^{2}\right)(i x)-T_{2 A}^{-3}-3 T_{2 A}^{-2} T_{1 X}^{-1}}= \\
& -2 T_{2 A}^{-1} T_{1 X}^{-2}-4 \pi^{2} J^{2}\left(T_{2 A}^{-1}+T_{1 X}^{-1}\right) \\
& =4 \frac{(i x)^{2}-(i x)\left(2 T_{2 A}^{-1}+3 T_{1 X}^{-1}\right)+T_{2 A}^{-2}+3 T_{2 A}^{-1} T_{1 X}^{-1}+2 T_{1 X}^{-2}+2 \pi^{2} J^{2}}{(i x)^{3}-3\left(T_{2 A}^{-1}+T_{1 X}^{-1}\right)(i x)^{2}+\left(3 T_{2 A}^{-2}+6 T_{2 A}^{-1} T_{1 X}^{-1}+2 T_{1 X}^{-2}+4 \pi^{2} J^{2}\right)(i x)-T_{2 A}^{-3}}= \\
& -3 T_{2 A}^{-2} T_{1 X}^{-1}-2 T_{2 A}^{-1} T_{1 X}^{-2}-4 \pi^{2} J^{2}\left(T_{2 A}^{-1}+T_{1 X}^{-1}\right) \\
& =4 \frac{P+Q(i x)}{R+S(i x)} \\
& P=-x^{2}+T_{2 A}^{-2}+3 T_{2 A}^{-1} T_{1 X}^{-1}+2 T_{1 X}^{-2}+2 \pi^{2} J^{2} \\
& Q=-2 T_{2 A}^{-1}-3 T_{1 X}^{-1} \\
& R=3\left(T_{2 A}^{-1}+T_{1 X}^{-1}\right) x^{2}-T_{2 A}^{-3}-3 T_{2 A}^{-2} T_{1 X}^{-1}-2 T_{2 A}^{-1} T_{1 X}^{-2}-4 \pi^{2} J^{2}\left(T_{2 A}^{-1}+T_{1 X}^{-1}\right) \\
& S=-x^{2}+3 T_{2 A}^{-2}+6 T_{2 A}^{-1} T_{1 X}^{-1}+2 T_{1 X}^{-2}+4 \pi^{2} J^{2} \\
& I(x)=4 \frac{P R+Q S x^{2}}{R^{2}+S^{2} x^{2}}(e q . S 3)
\end{aligned}
$$


Approximate solution around $\mathrm{x}=0$ :

$$
\begin{aligned}
& 4 / f(i x)=\frac{(i x)^{2}-(i x)\left(2 T_{2 A}^{-1}+3 T_{1 X}^{-1}\right)+T_{2 A}^{-2}+3 T_{2 A}^{-1} T_{1 X}^{-1}+2 T_{1 X}^{-2}+2 \pi^{2} J^{2}}{(i x)^{3}-3\left(T_{2 A}^{-1}+T_{1 X}^{-1}\right)(i x)^{2}+\left(3 T_{2 A}^{-2}+6 T_{2 A}^{-1} T_{1 X}^{-1}+2 T_{1 X}^{-2}+4 \pi^{2} J^{2}\right)(i x)-T_{2 A}^{-3}} \\
& -3 T_{2 A}^{-2} T_{1 X}^{-1}-2 T_{2 A}^{-1} T_{1 X}^{-2}-4 \pi^{2} J^{2}\left(T_{2 A}^{-1}+T_{1 X}^{-1}\right) \\
& f(0)=-\frac{T_{2 A}^{-3}+3 T_{2 A}^{-2} T_{1 X}^{-1}+2 T_{2 A}^{-1} T_{1 X}^{-2}+4 \pi^{2} J^{2}\left(T_{2 A}^{-1}+T_{1 X}^{-1}\right)}{T_{2 A}^{-2}+3 T_{2 A}^{-1} T_{1 X}^{-1}+2 T_{1 X}^{-2}+2 \pi^{2} J^{2}} \\
& \left(T_{2 A}^{-2}+3 T_{2 A}^{-1} T_{1 X}^{-1}+2 T_{1 X}^{-2}+2 \pi^{2} J^{2}\right)\left(3 T_{2 A}^{-2}+6 T_{2 A}^{-1} T_{1 X}^{-1}+2 T_{1 X}^{-2}+4 \pi^{2} J^{2}\right) \\
& f^{\prime}(0)=\frac{-\left(2 T_{2 A}^{-1}+3 T_{1 X}^{-1}\right)\left(T_{2 A}^{-3}+3 T_{2 A}^{-2} T_{1 X}^{-1}+2 T_{2 A}^{-1} T_{1 X}^{-2}+4 \pi^{2} J^{2}\left(T_{2 A}^{-1}+T_{1 X}^{-1}\right)\right)}{\left(T_{2 A}^{-2}+3 T_{2 A}^{-1} T_{1 X}^{-1}+2 T_{1 X}^{-2}+2 \pi^{2} J^{2}\right)^{2}}= \\
& =\frac{4 T_{1 X}^{-4}+12 T_{1 X}^{-3} T_{2 A}^{-1}+13 T_{1 X}^{-2} T_{2 A}^{-2}+T_{1 X}^{-1}\left(6 T_{2 A}^{-3}+4 T_{2 A}^{-1} \pi^{2} J^{2}\right)+T_{2 A}^{-4}+2 T_{2 A}^{-2} \pi^{2} J^{2}+8 \pi^{4} J^{4}}{\left(T_{2 A}^{-2}+3 T_{2 A}^{-1} T_{1 X}^{-1}+2 T_{1 X}^{-2}+2 \pi^{2} J^{2}\right)^{2}} \\
& \Rightarrow \\
& \left.f(i x) \approx \text { const. } \text {. ix }-\frac{f(0)}{f^{\prime}(0)}\right) \approx \text { const. }(\text { ix }-\pi W) \\
& \pi W \approx \frac{\left(T_{2 A}^{-3}+3 T_{2 A}^{-2} T_{1 X}^{-1}+2 T_{2 A}^{-1} T_{1 X}^{-2}+4 \pi^{2} J^{2}\left(T_{2 A}^{-1}+T_{1 X}^{-1}\right)\left(T_{2 A}^{-2}+3 T_{2 A}^{-1} T_{1 X}^{-1}+2 T_{1 X}^{-2}+2 \pi^{2} J^{2}\right)\right.}{4 T_{1 X}^{-4}+12 T_{1 X}^{-3} T_{2 A}^{-1}+13 T_{1 X}^{-2} T_{2 A}^{-2}+T_{1 X}^{-1}\left(6 T_{2 A}^{-3}+4 T_{2 A}^{-1} \pi^{2} J^{2}\right)+T_{2 A}^{-4}+2 T_{2 A}^{-2} \pi^{2} J^{2}+8 \pi^{4} J^{4}}= \\
& 4 T_{1 X}^{-4} T_{2 A}^{-1}+T_{1 X}^{-3}\left(12 T_{2 A}^{-2}+8 \pi^{2} J^{2}\right)+T_{1 X}^{-2}\left(13 T_{2 A}^{-3}+24 T_{2 A}^{-1} \pi^{2} J^{2}\right) \\
& =\frac{+T_{1 X}^{-1}\left(6 T_{2 A}^{-4}+22 T_{2 A}^{-2} \pi^{2} J^{2}+8 \pi^{4} J^{4}\right)+T_{2 A}^{-5}+6 T_{2 A}^{-3} \pi^{2} J^{2}+8 T_{2 A}^{-1} \pi^{4} J^{4}}{4 T_{1 X}^{-4}+12 T_{1 X}^{-3} T_{2 A}^{-1}+13 T_{1 X}^{-2} T_{2 A}^{-2}+T_{1 X}^{-1}\left(6 T_{2 A}^{-3}+4 T_{2 A}^{-1} \pi^{2} J^{2}\right)+T_{2 A}^{-4}+2 T_{2 A}^{-2} \pi^{2} J^{2}+8 \pi^{4} J^{4}}= \\
& 4 T_{2 A}^{-1}+T_{1 X}\left(12 T_{2 A}^{-2}+8 \pi^{2} J^{2}\right)+T_{1 X}^{2}\left(13 T_{2 A}^{-3}+24 T_{2 A}^{-1} \pi^{2} J^{2}\right) \\
& =\frac{+T_{1 X}^{3}\left(6 T_{2 A}^{-4}+22 T_{2 A}^{-2} \pi^{2} J^{2}+8 \pi^{4} J^{4}\right)+T_{1 X}^{4}\left(T_{2 A}^{-5}+6 T_{2 A}^{-3} \pi^{2} J^{2}+8 T_{2 A}^{-1} \pi^{4} J^{4}\right)}{4+12 T_{1 X} T_{2 A}^{-1}+13 T_{1 X}^{2} T_{2 A}^{-2}+T_{1 X}^{3}\left(6 T_{2 A}^{-3}+4 T_{2 A}^{-1} \pi^{2} J^{2}\right)+T_{1 X}^{4}\left(T_{2 A}^{-4}+2 T_{2 A}^{-2} \pi^{2} J^{2}+8 \pi^{4} J^{4}\right)}= \\
& 4 T_{1 X}^{-4} T_{2 A}^{-1}+12 T_{1 X}^{-3} T_{2 A}^{-2}+13 T_{1 X}^{-2} T_{2 A}^{-3}+6 T_{1 X}^{-1} T_{2 A}^{-4}+T_{2 A}^{-5} \\
& =\frac{+\left(8 T_{1 X}^{-3}+24 T_{1 X}^{-2} T_{2 A}^{-1}+22 T_{1 X}^{-1} T_{2 A}^{-2}+6 T_{2 A}^{-3}\right) \pi^{2} J^{2}+8\left(T_{1 X}^{-1}+T_{2 A}^{-1}\right) \pi^{4} J^{4}}{4 T_{1 X}^{-4}+12 T_{1 X}^{-3} T_{2 A}^{-1}+13 T_{1 X}^{-2} T_{2 A}^{-2}+6 T_{1 X}^{-1} T_{2 A}^{-3}+T_{2 A}^{-4}+\left(4 T_{1 X}^{-1} T_{2 A}^{-1}+2 T_{2 A}^{-2}\right) \pi^{2} J^{2}+8 \pi^{4} J^{4}}= \\
& 4 T_{2 A}^{-1}\left(T_{1 X}^{-1}+T_{2 A}^{-1}\right)^{4}-4 T_{2 A}^{-2}\left(T_{1 X}^{-1}+T_{2 A}^{-1}\right)^{3}+2 T_{2 A}^{-3}\left(T_{1 X}^{-1}+T_{2 A}^{-1}\right)^{2} \\
& =\frac{+\left(8\left(T_{1 X}^{-1}+T_{2 A}^{-1}\right)^{3}-2 T_{2 A}^{-2}\left(T_{1 X}^{-1}+T_{2 A}^{-1}\right)\right) \pi^{2} J^{2}+8\left(T_{1 X}^{-1}+T_{2 A}^{-1}\right) \pi^{4} J^{4}}{4\left(T_{1 X}^{-1}+T_{2 A}^{-1}\right)^{4}-4 T_{2 A}^{-1}\left(T_{1 X}^{-1}+T_{2 A}^{-1}\right)^{3}+2 T_{2 A}^{-2}\left(T_{1 X}^{-1}+T_{2 A}^{-1}\right)^{2}+\left(4 T_{1 X}^{-1} T_{2 A}^{-1}+2 T_{2 A}^{-2}\right) \pi^{2} J^{2}+8 \pi^{4} J^{4}}
\end{aligned}
$$


Taylor expansion in $\pi^{2} \mathrm{~J}^{2}$ :

$$
\begin{aligned}
\pi W \approx & T_{2 A}^{-1} \\
& \left(4 T_{1 X}^{-4}+12 T_{1 X}^{-3} T_{2 A}^{-1}+13 T_{1 X}^{-2} T_{2 A}^{-2}+6 T_{1 X}^{-1} T_{2 A}^{-3}+T_{2 A}^{-4}\right)\left(8 T_{1 X}^{-3}+24 T_{1 X}^{-2} T_{2 A}^{-1}+22 T_{1 X}^{-1} T_{2 A}^{-2}+6 T_{2 A}^{-3}\right)- \\
+ & \frac{\left(4 T_{1 X}^{-1} T_{2 A}^{-1}+2 T_{2 A}^{-2}\right)\left(4 T_{1 X}^{-4}+12 T_{1 X}^{-3} T_{2 A}^{-1}+13 T_{1 X}^{-2} T_{2 A}^{-2}+6 T_{1 X}^{-1} T_{2 A}^{-3}+T_{2 A}^{-4}\right) T_{2 A}^{-1}}{\left(4 T_{1 X}^{-4}+12 T_{1 X}^{-3} T_{2 A}^{-1}+13 T_{1 X}^{-2} T_{2 A}^{-2}+6 T_{1 X}^{-1} T_{2 A}^{-3}+T_{2 A}^{-4}\right)^{2}} \pi^{2} J^{2}= \\
& \left(4 T_{1 X}^{-4}+12 T_{1 X}^{-3} T_{2 A}^{-1}+13 T_{1 X}^{-2} T_{2 A}^{-2}+6 T_{1 X}^{-1} T_{2 A}^{-3}+T_{2 A}^{-4}\right) \\
= & T_{2 A}^{-1}+\frac{\left(8 T_{1 X}^{-3}+24 T_{1 X}^{-2} T_{2 A}^{-1}+22 T_{1 X}^{-1} T_{2 A}^{-2}+6 T_{2 A}^{-3}-4 T_{1 X}^{-1} T_{2 A}^{-2}-2 T_{2 A}^{-3}\right)}{\left(4 T_{1 X}^{-4}+12 T_{1 X}^{-3} T_{2 A}^{-1}+13 T_{1 X}^{-2} T_{2 A}^{-2}+6 T_{1 X}^{-1} T_{2 A}^{-3}+T_{2 A}^{-4}\right)^{2}} \pi^{2} J^{2}= \\
= & T_{2 A}^{-1}+\frac{8 T_{1 X}^{-3}+24 T_{1 X}^{-2} T_{2 A}^{-1}+18 T_{1 X}^{-1} T_{2 A}^{-2}+4 T_{2 A}^{-3}}{4 T_{1 X}^{-4}+12 T_{1 X}^{-3} T_{2 A}^{-1}+13 T_{1 X}^{-2} T_{2 A}^{-2}+6 T_{1 X}^{-1} T_{2 A}^{-3}+T_{2 A}^{-4}} \pi^{2} J^{2}= \\
= & T_{2 A}^{-1}+\frac{2\left(2 T_{1 X}^{-1}+T_{2 A}^{-1}\right)^{2}\left(T_{1 X}^{-1}+2 T_{2 A}^{-1}\right)}{\left(2 T_{1 X}^{-1}+T_{2 A}^{-1}\right)^{2}\left(T_{1 X}^{-1}+T_{2 A}^{-1}\right)^{2}} \pi^{2} J^{2}=T_{2 A}^{-1}+2 \frac{T_{1 X}^{-1}+2 T_{2 A}^{-1}}{\left(T_{1 X}^{-1}+T_{2 A}^{-1}\right)^{2}} \pi^{2} J^{2}
\end{aligned}
$$

Again, this approximation over-estimates the width. 


\section{Quartet Case:}

$A=\left[\begin{array}{cccc}i x+3 \pi J i-T_{2 A}^{-1}-\frac{3}{2} T_{1 X}^{-1} & \frac{3}{2} T_{1 X}^{-1} & 0 & 0 \\ \frac{1}{2} T_{1 X}^{-1} & i x+\pi J i-T_{2 A}^{-1}-\frac{3}{2} T_{1 X}^{-1} & T_{1 X}^{-1} & 0 \\ 0 & T_{1 X}^{-1} & i x-\pi J i-T_{2 A}^{-1}-\frac{3}{2} T_{1 X}^{-1} & \frac{1}{2} T_{1 X}^{-1} \\ 0 & 0 & \frac{3}{2} T_{1 X}^{-1} & i x-3 \pi J i-T_{2 A}^{-1}-\frac{3}{2} T_{1 X}^{-1}\end{array}\right]$

$x=2 \pi\left(v-v_{0}\right)$

$|A|=\left(i x-T_{2 A}^{-1}-\frac{3}{2} T_{1 X}^{-1}+3 \pi J i\right)\left(\left(i x-T_{2 A}^{-1}-\frac{3}{2} T_{1 X}^{-1}-3 \pi J i\right)\left(\left(i x-T_{2 A}^{-1}-\frac{3}{2} T_{1 X}^{-1}\right)^{2}+\pi^{2} J^{2}-T_{1 X}^{-2}\right)\right.$

$\left.-\frac{3}{4} T_{1 X}^{-2}\left(i x-T_{2 A}^{-1}-\frac{3}{2} T_{1 X}^{-1}+\pi J i\right)\right)-\frac{3}{4} T_{1 X}^{-2}\left(\left(i x-T_{2 A}^{-1}-\frac{3}{2} T_{1 X}^{-1}-\pi J i\right)\left(i x-T_{2 A}^{-1}-\frac{3}{2} T_{1 X}^{-1}-3 \pi J i\right)-\frac{3}{4} T_{1 X}^{-2}\right)=$

$=\left(i x-T_{2 A}^{-1}-\frac{3}{2} T_{1 X}^{-1}+3 \pi J i\right)\left(\left(i x-T_{2 A}^{-1}-\frac{3}{2} T_{1 X}^{-1}\right)^{3}+\left(\pi^{2} J^{2}-T_{1 X}^{-2}\right)\left(i x-T_{2 A}^{-1}-\frac{3}{2} T_{1 X}^{-1}\right)\right.$

$\left.-3 \pi J i\left(i x-T_{2 A}^{-1}-\frac{3}{2} T_{1 X}^{-1}\right)^{2}-3 \pi J i\left(\pi^{2} J^{2}-T_{1 X}^{-2}\right)-\frac{3}{4} T_{1 X}^{-2}\left(i x-T_{2 A}^{-1}-\frac{3}{2} T_{1 X}^{-1}\right)-\frac{3}{4} T_{1 X}^{-2} \pi J i\right)$

$-\frac{3}{4} T_{1 X}^{-2}\left(\left(i x-T_{2 A}^{-1}-\frac{3}{2} T_{1 X}^{-1}\right)^{2}-4 \pi J i\left(i x-T_{2 A}^{-1}-\frac{3}{2} T_{1 X}^{-1}\right)-3 \pi^{2} J^{2}-\frac{3}{4} T_{1 X}^{-2}\right)=$

$=\left(i x-T_{2 A}^{-1}-\frac{3}{2} T_{1 X}^{-1}\right)^{4}+\left(\pi^{2} J^{2}-T_{1 X}^{-2}\right)\left(i x-T_{2 A}^{-1}-\frac{3}{2} T_{1 X}^{-1}\right)^{2}-3 \pi J i\left(i x-T_{2 A}^{-1}-\frac{3}{2} T_{1 X}^{-1}\right)^{3}$

$-3 \pi J i\left(\pi^{2} J^{2}-T_{1 X}^{-2}\right)\left(i x-T_{2 A}^{-1}-\frac{3}{2} T_{1 X}^{-1}\right)-\frac{3}{4} T_{1 X}^{-2}\left(i x-T_{2 A}^{-1}-\frac{3}{2} T_{1 X}^{-1}\right)^{2}-\frac{3}{4} T_{1 X}^{-2} \pi J i\left(i x-T_{2 A}^{-1}-\frac{3}{2} T_{1 X}^{-1}\right)$

$+3 \pi J i\left(i x-T_{2 A}^{-1}-\frac{3}{2} T_{1 X}^{-1}\right)^{3}+3 \pi J i\left(\pi^{2} J^{2}-T_{1 X}^{-2}\right)\left(i x-T_{2 A}^{-1}-\frac{3}{2} T_{1 X}^{-1}\right)+9 \pi^{2} J^{2}\left(i x-T_{2 A}^{-1}-\frac{3}{2} T_{1 X}^{-1}\right)^{2}$

$+9 \pi^{2} J^{2}\left(\pi^{2} J^{2}-T_{1 X}^{-2}\right)-\frac{9}{4} T_{1 X}^{-2}\left(i x-T_{2 A}^{-1}-\frac{3}{2} T_{1 X}^{-1}\right) \pi J i+\frac{9}{4} T_{1 X}^{-2} \pi^{2} J^{2}-\frac{3}{4} T_{1 X}^{-2}\left(i x-T_{2 A}^{-1}-\frac{3}{2} T_{1 X}^{-1}\right)^{2}$

$+3 T_{1 X}^{-2} \pi J i\left(i x-T_{2 A}^{-1}-\frac{3}{2} T_{1 X}^{-1}\right)+\frac{9}{4} T_{1 X}^{-2} \pi^{2} J^{2}+\frac{9}{16} T_{1 X}^{-4}=$

$=\left(i x-T_{2 A}^{-1}-\frac{3}{2} T_{1 X}^{-1}\right)^{4}+(-3 \pi J i+3 \pi J i)\left(i x-T_{2 A}^{-1}-\frac{3}{2} T_{1 X}^{-1}\right)^{3}$

$+\left(\pi^{2} J^{2}-T_{1 X}^{-2}-\frac{3}{4} T_{1 X}^{-2}+9 \pi^{2} J^{2}-\frac{3}{4} T_{1 X}^{-2}\right)\left(i x-T_{2 A}^{-1}-\frac{3}{2} T_{1 X}^{-1}\right)^{2}$

$+\left(-3 \pi J i \pi^{2} J^{2}+3 \pi J i T_{1 X}^{-2}-\frac{3}{4} T_{1 X}^{-2} \pi J i+3 \pi J i \pi^{2} J^{2}-3 \pi J i T_{1 X}^{-2}-\frac{9}{4} \pi J i T_{1 X}^{-2}+3 T_{1 X}^{-2} \pi J i\right)\left(i x-T_{2 A}^{-1}-\frac{3}{2} T_{1 X}^{-1}\right)$

$+9 \pi^{4} J^{4}-9 \pi^{2} J^{2} T_{1 X}^{-2}+\frac{9}{4} T_{1 X}^{-2} \pi^{2} J^{2}+\frac{9}{4} T_{1 X}^{-2} \pi^{2} J^{2}+\frac{9}{16} T_{1 X}^{-4}=$

$=\left(i x-T_{2 A}^{-1}-\frac{3}{2} T_{1 X}^{-1}\right)^{4}+\left(10 \pi^{2} J^{2}-\frac{5}{2} T_{1 X}^{-2}\right)\left(i x-T_{2 A}^{-1}-\frac{3}{2} T_{1 X}^{-1}\right)^{2}+9 \pi^{4} J^{4}-\frac{9}{2} T_{1 X}^{-2} \pi^{2} J^{2}+\frac{9}{16} T_{1 X}^{-4}=$

$=(i x)^{4}-(i x)^{3}\left(4 T_{2 A}^{-1}+6 T_{1 X}^{-1}\right)+(i x)^{2}\left(6 T_{2 A}^{-2}+18 T_{2 A}^{-1} T_{1 X}^{-1}+\frac{27}{2} T_{1 X}^{-2}+10 \pi^{2} J^{2}-\frac{5}{2} T_{1 X}^{-2}\right)$

$-(i x)\left(4 T_{2 A}^{-3}+18 T_{2 A}^{-2} T_{1 X}^{-1}+27 T_{2 A}^{-1} T_{1 X}^{-2}+\frac{27}{2} T_{1 X}^{-3}+20 T_{2 A}^{-1} \pi^{2} J^{2}+30 T_{1 X}^{-1} \pi^{2} J^{2}-5 T_{2 A}^{-1} T_{1 X}^{-2}-\frac{15}{2} T_{1 X}^{-3}\right)$

$+T_{2 A}^{-4}+6 T_{2 A}^{-3} T_{1 X}^{-1}+\frac{27}{2} T_{2 A}^{-2} T_{1 X}^{-2}+\frac{27}{2} T_{2 A}^{-1} T_{1 X}^{-3}+\frac{81}{16} T_{1 X}^{-4}-\frac{5}{2} T_{2 A}^{-2} T_{1 X}^{-2}-\frac{15}{2} T_{2 A}^{-1} T_{1 X}^{-3}-\frac{45}{8} T_{1 X}^{-4}+\frac{9}{16} T_{1 X}^{-4}$

$+10 T_{2 A}^{-2} \pi^{2} J^{2}+30 T_{2 A}^{-1} T_{1 X}^{-1} \pi^{2} J^{2}+\frac{45}{2} T_{1 X}^{-2} \pi^{2} J^{2}+9 \pi^{4} J^{4}-\frac{9}{2} T_{1 X}^{-2} \pi^{2} J^{2}=$

$=(i x)^{4}-(i x)^{3}\left(4 T_{2 A}^{-1}+6 T_{1 X}^{-1}\right)+(i x)^{2}\left(6 T_{2 A}^{-2}+18 T_{2 A}^{-1} T_{1 X}^{-1}+11 T_{1 X}^{-2}+10 \pi^{2} J^{2}\right)$

$-(i x)\left(4 T_{2 A}^{-3}+18 T_{2 A}^{-2} T_{1 X}^{-1}+22 T_{2 A}^{-1} T_{1 X}^{-2}+6 T_{1 X}^{-3}+20 T_{2 A}^{-1} \pi^{2} J^{2}+30 T_{1 X}^{-1} \pi^{2} J^{2}\right)$

$+T_{2 A}^{-4}+6 T_{2 A}^{-3} T_{1 X}^{-1}+11 T_{2 A}^{-2} T_{1 X}^{-2}+6 T_{2 A}^{-1} T_{1 X}^{-3}+10 T_{2 A}^{-2} \pi^{2} J^{2}+30 T_{2 A}^{-1} T_{1 X}^{-1} \pi^{2} J^{2}+18 T_{1 X}^{-2} \pi^{2} J^{2}+9 \pi^{4} J^{4}$ 


$$
A=\left[\begin{array}{cccc}
i x+3 \pi J i-T_{2 A}^{-1}-\frac{3}{2} T_{1 X}^{-1} & \frac{3}{2} T_{1 X}^{-1} & 0 & 0 \\
\frac{1}{2} T_{1 X}^{-1} & i x+\pi J i-T_{2 A}^{-1}-\frac{3}{2} T_{1 X}^{-1} & T_{1 X}^{-1} & 0 \\
0 & T_{1 X}^{-1} & i x-\pi J i-T_{2 A}^{-1}-\frac{3}{2} T_{1 X}^{-1} & \frac{1}{2} T_{1 X}^{-1} \\
0 & 0 & \frac{3}{2} T_{1 X}^{-1} & i x-3 \pi J i-T_{2 A}^{-1}-\frac{3}{2} T_{1 X}^{-1}
\end{array}\right]
$$

$$
\begin{aligned}
& A^{-1}= \\
& =\frac{\left[\begin{array}{llll}
A_{11} & A_{12} & A_{13} & A_{14} \\
A_{21} & A_{22} & A_{23} & A_{24} \\
A_{31} & A_{32} & A_{33} & A_{34} \\
A_{41} & A_{42} & A_{43} & A_{44}
\end{array}\right]}{|A|}
\end{aligned}
$$

$$
\begin{aligned}
& I(x)=\operatorname{Re}\left\{W \bullet A^{-1} \bullet 1\right\} \\
& W \bullet A^{-1} \bullet 1=\left(\begin{array}{l}
1 \\
3 \\
3 \\
1
\end{array}\right) \bullet A^{-1} \bullet\left(\begin{array}{l}
1 \\
1 \\
1 \\
1
\end{array}\right)= \\
& =\frac{A_{11}+A_{44}+A_{12}+A_{43}+A_{13}+A_{42}+A_{14}+A_{41}+3\left(A_{21}+A_{34}+A_{22}+A_{33}+A_{23}+A_{32}+A_{24}+A_{31}\right)}{|A|}
\end{aligned}
$$




$$
\begin{aligned}
& A_{11}=\left(i x-3 \pi J i-T_{2 A}^{-1}-\frac{3}{2} T_{1 X}^{-1}\right)\left(\left(i x-T_{2 A}^{-1}-\frac{3}{2} T_{1 X}^{-1}\right)^{2}+\pi^{2} J^{2}-T_{1 X}^{-2}\right) \\
& -\frac{3}{4} T_{1 X}^{-2}\left(i x+\pi J i-T_{2 A}^{-1}-\frac{3}{2} T_{1 X}^{-1}\right)= \\
& =\left(i x-T_{2 A}^{-1}-\frac{3}{2} T_{1 X}^{-1}\right)^{3}+\left(\pi^{2} J^{2}-T_{1 X}^{-2}\right)\left(i x-T_{2 A}^{-1}-\frac{3}{2} T_{1 X}^{-1}\right)-3 \pi J i\left(i x-T_{2 A}^{-1}-\frac{3}{2} T_{1 X}^{-1}\right)^{2} \\
& -3 \pi^{3} J^{3} i+3 \pi J i T_{1 X}^{-2}-\frac{3}{4} T_{1 X}^{-2}\left(i x-T_{2 A}^{-1}-\frac{3}{2} T_{1 X}^{-1}\right)-\frac{3}{4} T_{1 X}^{-2} \pi J i= \\
& =\left(i x-T_{2 A}^{-1}-\frac{3}{2} T_{1 X}^{-1}\right)^{3}-3 \pi J i\left(i x-T_{2 A}^{-1}-\frac{3}{2} T_{1 X}^{-1}\right)^{2}+\left(\pi^{2} J^{2}-\frac{7}{4} T_{1 X}^{-2}\right)\left(i x-T_{2 A}^{-1}-\frac{3}{2} T_{1 X}^{-1}\right) \\
& -3 \pi^{3} J^{3} i+\frac{9}{4} \pi J i T_{1 X}^{-2}
\end{aligned}
$$$$
A_{12}=-\frac{3}{2} T_{1 X}^{-1}\left(\left(i x-\pi J i-T_{2 A}^{-1}-\frac{3}{2} T_{1 X}^{-1}\right)\left(i x-3 \pi J i-T_{2 A}^{-1}-\frac{3}{2} T_{1 X}^{-1}\right)-\frac{3}{4} T_{1 X}^{-2}\right)=
$$$$
-\frac{3}{2} T_{1 X}^{-1}\left(i x-T_{2 A}^{-1}-\frac{3}{2} T_{1 X}^{-1}\right)^{2}+6 T_{1 X}^{-1} \pi J i\left(i x-T_{2 A}^{-1}-\frac{3}{2} T_{1 X}^{-1}\right)+\frac{9}{2} \pi^{2} J^{2} T_{1 X}^{-1}+\frac{9}{8} T_{1 X}^{-3}
$$$$
A_{13}=\frac{3}{2} T_{1 X}^{-2}\left(i x-3 \pi J i-T_{2 A}^{-1}-\frac{3}{2} T_{1 X}^{-1}\right)=\frac{3}{2} T_{1 X}^{-2}\left(i x-T_{2 A}^{-1}-\frac{3}{2} T_{1 X}^{-1}\right)-\frac{9}{2} T_{1 X}^{-2} \pi J i
$$$$
A_{14}=-\frac{3}{4} T_{1 X}^{-3}
$$$$
A_{21}=-\frac{1}{2} T_{1 X}^{-1}\left(\left(i x-\pi J i-T_{2 A}^{-1}-\frac{3}{2} T_{1 X}^{-1}\right)\left(i x-3 \pi J i-T_{2 A}^{-1}-\frac{3}{2} T_{1 X}^{-1}\right)-\frac{3}{4} T_{1 X}^{-2}\right)=
$$$$
-\frac{1}{2} T_{1 X}^{-1}\left(i x-T_{2 A}^{-1}-\frac{3}{2} T_{1 X}^{-1}\right)^{2}+2 T_{1 X}^{-1} \pi J i\left(i x-T_{2 A}^{-1}-\frac{3}{2} T_{1 X}^{-1}\right)+\frac{3}{2} \pi^{2} J^{2} T_{1 X}^{-1}+\frac{3}{8} T_{1 X}^{-3}
$$$$
A_{22}=\left(i x-\pi J i-T_{2 A}^{-1}-\frac{3}{2} T_{1 X}^{-1}\right)\left(\left(i x-T_{2 A}^{-1}-\frac{3}{2} T_{1 X}^{-1}\right)^{2}+9 \pi^{2} J^{2}\right)-\frac{3}{4} T_{1 X}^{-2}\left(i x+3 \pi J i-T_{2 A}^{-1}-\frac{3}{2} T_{1 X}^{-1}\right)=
$$$$
=\left(i x-T_{2 A}^{-1}-\frac{3}{2} T_{1 X}^{-1}\right)^{3}+9 \pi^{2} J^{2}\left(i x-T_{2 A}^{-1}-\frac{3}{2} T_{1 X}^{-1}\right)-\pi J i\left(i x-T_{2 A}^{-1}-\frac{3}{2} T_{1 X}^{-1}\right)^{2}-9 \pi^{3} J^{3} i
$$$$
-\frac{3}{4} T_{1 X}^{-2}\left(i x-T_{2 A}^{-1}-\frac{3}{2} T_{1 X}^{-1}\right)-\frac{9}{4} T_{1 X}^{-2} \pi J i=
$$$$
=\left(i x-T_{2 A}^{-1}-\frac{3}{2} T_{1 X}^{-1}\right)^{3}-\pi J i\left(i x-T_{2 A}^{-1}-\frac{3}{2} T_{1 X}^{-1}\right)^{2}+\left(9 \pi^{2} J^{2}-\frac{3}{4} T_{1 X}^{-2}\right)\left(i x-T_{2 A}^{-1}-\frac{3}{2} T_{1 X}^{-1}\right)
$$$$
-9 \pi^{3} J^{3} i-\frac{9}{4} \pi J i T_{1 X}^{-2}
$$$$
A_{23}=-T_{1 X}^{-1}\left(\left(i x-T_{2 A}^{-1}-\frac{3}{2} T_{1 X}^{-1}\right)^{2}+9 \pi^{2} J^{2}\right)=-T_{1 X}^{-1}\left(i x-T_{2 A}^{-1}-\frac{3}{2} T_{1 X}^{-1}\right)^{2}-9 T_{1 X}^{-1} \pi^{2} J^{2}
$$$$
A_{24}=\frac{1}{2} T_{1 X}^{-2}\left(i x+3 \pi J i-T_{2 A}^{-1}-\frac{3}{2} T_{1 X}^{-1}\right)=\frac{1}{2} T_{1 X}^{-2}\left(i x-T_{2 A}^{-1}-\frac{3}{2} T_{1 X}^{-1}\right)+\frac{3}{2} T_{1 X}^{-2} \pi J i
$$

$$
\begin{aligned}
& A_{31}=\frac{1}{2} T_{1 X}^{-2}\left(i x-3 \pi J i-T_{2 A}^{-1}-\frac{3}{2} T_{1 X}^{-1}\right)=\frac{1}{2} T_{1 X}^{-2}\left(i x-T_{2 A}^{-1}-\frac{3}{2} T_{1 X}^{-1}\right)-\frac{3}{2} T_{1 X}^{-2} \pi J i \\
& A_{32}=-T_{1 X}^{-1}\left(\left(i x-T_{2 A}^{-1}-\frac{3}{2} T_{1 X}^{-1}\right)^{2}+9 \pi^{2} J^{2}\right)=-T_{1 X}^{-1}\left(i x-T_{2 A}^{-1}-\frac{3}{2} T_{1 X}^{-1}\right)^{2}-9 T_{1 X}^{-1} \pi^{2} J^{2} \\
& A_{33}=\left(i x+\pi J i-T_{2 A}^{-1}-\frac{3}{2} T_{1 X}^{-1}\right)\left(\left(i x-T_{2 A}^{-1}-\frac{3}{2} T_{1 X}^{-1}\right)^{2}+9 \pi^{2} J^{2}\right)-\frac{3}{4} T_{1 X}^{-2}\left(i x-3 \pi J i-T_{2 A}^{-1}-\frac{3}{2} T_{1 X}^{-1}\right)= \\
& =\left(i x-T_{2 A}^{-1}-\frac{3}{2} T_{1 X}^{-1}\right)^{3}+9 \pi^{2} J^{2}\left(i x-T_{2 A}^{-1}-\frac{3}{2} T_{1 X}^{-1}\right)+\pi J i\left(i x-T_{2 A}^{-1}-\frac{3}{2} T_{1 X}^{-1}\right)^{2}+9 \pi^{3} J^{3} i \\
& -\frac{3}{4} T_{1 X}^{-2}\left(i x-T_{2 A}^{-1}-\frac{3}{2} T_{1 X}^{-1}\right)+\frac{9}{4} T_{1 X}^{-2} \pi J i= \\
& =\left(i x-T_{2 A}^{-1}-\frac{3}{2} T_{1 X}^{-1}\right)^{3}+\pi J i\left(i x-T_{2 A}^{-1}-\frac{3}{2} T_{1 X}^{-1}\right)^{2}+\left(9 \pi^{2} J^{2}-\frac{3}{4} T_{1 X}^{-2}\right)\left(i x-T_{2 A}^{-1}-\frac{3}{2} T_{1 X}^{-1}\right) \\
& +9 \pi^{3} J^{3} i+\frac{9}{4} \pi J i T_{1 X}^{-2} \\
& A_{34}=-\frac{1}{2} T_{1 X}^{-1}\left(\left(i x+\pi J i-T_{2 A}^{-1}-\frac{3}{2} T_{1 X}^{-1}\right)\left(i x+3 \pi J i-T_{2 A}^{-1}-\frac{3}{2} T_{1 X}^{-1}\right)-\frac{3}{4} T_{1 X}^{-2}\right)= \\
& -\frac{1}{2} T_{1 X}^{-1}\left(i x-T_{2 A}^{-1}-\frac{3}{2} T_{1 X}^{-1}\right)^{2}-2 T_{1 X}^{-1} \pi J i\left(i x-T_{2 A}^{-1}-\frac{3}{2} T_{1 X}^{-1}\right)+\frac{3}{2} \pi^{2} J^{2} T_{1 X}^{-1}+\frac{3}{8} T_{1 X}^{-3}
\end{aligned}
$$




$$
\begin{aligned}
& A_{41}=-\frac{3}{4} T_{1 X}^{-3} \\
& A_{42}=\frac{3}{2} T_{1 X}^{-2}\left(i x+3 \pi J i-T_{2 A}^{-1}-\frac{3}{2} T_{1 X}^{-1}\right)=\frac{3}{2} T_{1 X}^{-2}\left(i x-T_{2 A}^{-1}-\frac{3}{2} T_{1 X}^{-1}\right)+\frac{9}{2} T_{1 X}^{-2} \pi J i \\
& A_{43}=-\frac{3}{2} T_{1 X}^{-1}\left(\left(i x+\pi J i-T_{2 A}^{-1}-\frac{3}{2} T_{1 X}^{-1}\right)\left(i x+3 \pi J i-T_{2 A}^{-1}-\frac{3}{2} T_{1 X}^{-1}\right)-\frac{3}{4} T_{1 X}^{-2}\right)= \\
& -\frac{3}{2} T_{1 X}^{-1}\left(i x-T_{2 A}^{-1}-\frac{3}{2} T_{1 X}^{-1}\right)^{2}-6 T_{1 X}^{-1} \pi J i\left(i x-T_{2 A}^{-1}-\frac{3}{2} T_{1 X}^{-1}\right)+\frac{9}{2} \pi^{2} J^{2} T_{1 X}^{-1}+\frac{9}{8} T_{1 X}^{-3} \\
& A_{44}=\left(i x+3 \pi J i-T_{2 A}^{-1}-\frac{3}{2} T_{1 X}^{-1}\right)\left(\left(i x-T_{2 A}^{-1}-\frac{3}{2} T_{1 X}^{-1}\right)^{2}+\pi^{2} J^{2}-T_{1 X}^{-2}\right) \\
& -\frac{3}{4} T_{1 X}^{-2}\left(i x-\pi J i-T_{2 A}^{-1}-\frac{3}{2} T_{1 X}^{-1}\right)= \\
& =\left(i x-T_{2 A}^{-1}-\frac{3}{2} T_{1 X}^{-1}\right)^{3}+\left(\pi^{2} J^{2}-T_{1 X}^{-2}\right)\left(i x-T_{2 A}^{-1}-\frac{3}{2} T_{1 X}^{-1}\right)+3 \pi J i\left(i x-T_{2 A}^{-1}-\frac{3}{2} T_{1 X}^{-1}\right)^{2} \\
& +3 \pi^{3} J^{3} i-3 \pi J i T_{1 X}^{-2}-\frac{3}{4} T_{1 X}^{-2}\left(i x-T_{2 A}^{-1}-\frac{3}{2} T_{1 X}^{-1}\right)+\frac{3}{4} T_{1 X}^{-2} \pi J i= \\
& =\left(i x-T_{2 A}^{-1}-\frac{3}{2} T_{1 X}^{-1}\right)^{3}+3 \pi J i\left(i x-T_{2 A}^{-1}-\frac{3}{2} T_{1 X}^{-1}\right)^{2}+\left(\pi^{2} J^{2}-\frac{7}{4} T_{1 X}^{-2}\right)\left(i x-T_{2 A}^{-1}-\frac{3}{2} T_{1 X}^{-1}\right) \\
& +3 \pi^{3} J^{3} i-\frac{9}{4} \pi J i T
\end{aligned}
$$

$$
\begin{aligned}
& A_{11}+A_{44}=2\left(i x-T_{2 A}^{-1}-\frac{3}{2} T_{1 X}^{-1}\right)^{3}+2\left(\pi^{2} J^{2}-\frac{7}{4} T_{1 X}^{-2}\right)\left(i x-T_{2 A}^{-1}-\frac{3}{2} T_{1 X}^{-1}\right) \\
& 3\left(A_{22}+A_{33}\right)=6\left(i x-T_{2 A}^{-1}-\frac{3}{2} T_{1 X}^{-1}\right)^{3}+6\left(9 \pi^{2} J^{2}-\frac{3}{4} T_{1 X}^{-2}\right)\left(i x-T_{2 A}^{-1}-\frac{3}{2} T_{1 X}^{-1}\right)
\end{aligned}
$$

$$
\begin{aligned}
& 3\left(A_{21}+A_{34}\right)=-3 T_{1 X}^{-1}\left(i x-T_{2 A}^{-1}-\frac{3}{2} T_{1 X}^{-1}\right)^{2}+9 \pi^{2} J^{2} T_{1 X}^{-1}+\frac{9}{4} T_{1 X}^{-3} \\
& 3\left(A_{23}+A_{32}\right)=-6 T_{1 X}^{-1}\left(i x-T_{2 A}^{-1}-\frac{3}{2} T_{1 X}^{-1}\right)^{2}-54 T_{1 X}^{-1} \pi^{2} J^{2} \\
& A_{12}+A_{43}=-3 T_{1 X}^{-1}\left(i x-T_{2 A}^{-1}-\frac{3}{2} T_{1 X}^{-1}\right)^{2}+9 \pi^{2} J^{2} T_{1 X}^{-1}+\frac{9}{4} T_{1 X}^{-3} \\
& A_{13}+A_{42}=3 T_{1 X}^{-2}\left(i x-T_{2 A}^{-1}-\frac{3}{2} T_{1 X}^{-1}\right) \\
& 3\left(A_{24}+A_{31}\right)=3 T_{1 X}^{-2}\left(i x-T_{2 A}^{-1}-\frac{3}{2} T_{1 X}^{-1}\right)
\end{aligned}
$$$$
A_{14}+A_{41}=-\frac{3}{2} T_{1 X}^{-3}
$$$$
\left(W \bullet A^{-1} \bullet 1\right)|A|=
$$$$
=A_{11}+A_{44}+A_{12}+A_{43}+A_{13}+A_{42}+A_{14}+A_{41}+3\left(A_{21}+A_{34}+A_{22}+A_{33}+A_{23}+A_{32}+A_{24}+A_{31}\right)=
$$$$
8\left(i x-T_{2 A}^{-1}-\frac{3}{2} T_{1 X}^{-1}\right)^{3}
$$$$
-12 T_{1 X}^{-1}\left(i x-T_{2 A}^{-1}-\frac{3}{2} T_{1 X}^{-1}\right)^{2}
$$$$
+\left(56 \pi^{2} J^{2}-2 T_{1 X}^{-2}\right)\left(i x-T_{2 A}^{-1}-\frac{3}{2} T_{1 X}^{-1}\right)
$$$$
-36 T_{1 X}^{-1} \pi^{2} J^{2}+3 T_{1 X}^{-3}=
$$ 


$$
\begin{aligned}
& =8(i x)^{3}-(i x)^{2}\left(24 T_{2 A}^{-1}+36 T_{1 X}^{-1}+12 T_{1 X}^{-1}\right) \\
& +(i x)\left(24 T_{2 A}^{-2}+72 T_{1 X}^{-1} T_{2 A}^{-1}+54 T_{1 X}^{-2}+24 T_{1 X}^{-1} T_{2 A}^{-1}+36 T_{1 X}^{-2}+56 \pi^{2} J^{2}-2 T_{1 X}^{-2}\right) \\
& -8 T_{2 A}^{-3}-36 T_{1 X}^{-1} T_{2 A}^{-2}-54 T_{1 X}^{-2} T_{2 A}^{-1}-27 T_{1 X}^{-3}-12 T_{1 X}^{-1} T_{2 A}^{-2}-36 T_{1 X}^{-2} T_{2 A}^{-1}-27 T_{1 X}^{-3}-56 \pi^{2} J^{2} T_{2 A}^{-1}+2 T_{2 A}^{-1} T_{1 X}^{-2} \\
& -84 \pi^{2} J^{2} T_{1 X}^{-1}+3 T_{1 X}^{-3}+3 T_{1 X}^{-3}-36 \pi^{2} J^{2} T_{1 X}^{-1}= \\
& =8(i x)^{3}-(i x)^{2}\left(24 T_{2 A}^{-1}+48 T_{1 X}^{-1}\right)+(i x)\left(24 T_{2 A}^{-2}+96 T_{1 X}^{-1} T_{2 A}^{-1}+88 T_{1 X}^{-2}+56 \pi^{2} J^{2}\right) \\
& -8 T_{2 A}^{-3}-48 T_{1 X}^{-1} T_{2 A}^{-2}-88 T_{1 X}^{-2} T_{2 A}^{-1}-48 T_{1 X}^{-3}-56 \pi^{2} J^{2} T_{2 A}^{-1}-120 \pi^{2} J^{2} T_{1 X}^{-1}= \\
& =8\left((i x)^{3}-3(i x)^{2}\left(T_{2 A}^{-1}+2 T_{1 X}^{-1}\right)+(i x)\left(3 T_{2 A}^{-2}+12 T_{1 X}^{-1} T_{2 A}^{-1}+11 T_{1 X}^{-2}+7 \pi^{2} J^{2}\right)\right. \\
& \left.-T_{2 A}^{-3}-6 T_{1 X}^{-1} T_{2 A}^{-2}-11 T_{1 X}^{-2} T_{2 A}^{-1}-6 T_{1 X}^{-3}-7 \pi^{2} J^{2} T_{2 A}^{-1}-15 \pi^{2} J^{2} T_{1 X}^{-1}\right) \\
& I(x)=\operatorname{Re}\left\{W \bullet A^{-1} \bullet 1\right\} \\
& W \bullet A^{-1} \bullet 1=8 / f(i x)
\end{aligned}
$$

$$
\begin{aligned}
& \quad(i x)^{3}-3(i x)^{2}\left(T_{2 A}^{-1}+2 T_{1 X}^{-1}\right)+(i x)\left(3 T_{2 A}^{-2}+12 T_{1 X}^{-1} T_{2 A}^{-1}+11 T_{1 X}^{-2}+7 \pi^{2} J^{2}\right) \\
& =8 \frac{-T_{2 A}^{-3}-6 T_{1 X}^{-1} T_{2 A}^{-2}-11 T_{1 X}^{-2} T_{2 A}^{-1}-6 T_{1 X}^{-3}-7 \pi^{2} J^{2} T_{2 A}^{-1}-15 \pi^{2} J^{2} T_{1 X}^{-1}}{(i x)^{4}-(i x)^{3}\left(4 T_{2 A}^{-1}+6 T_{1 X}^{-1}\right)+(i x)^{2}\left(6 T_{2 A}^{-2}+18 T_{2 A}^{-1} T_{1 X}^{-1}+11 T_{1 X}^{-2}+10 \pi^{2} J^{2}\right)}= \\
& \quad-(i x)\left(4 T_{2 A}^{-3}+18 T_{2 A}^{-2} T_{1 X}^{-1}+22 T_{2 A}^{-1} T_{1 X}^{-2}+6 T_{1 X}^{-3}+20 T_{2 A}^{-1} \pi^{2} J^{2}+30 T_{1 X}^{-1} \pi^{2} J^{2}\right) \\
& \quad+T_{2 A}^{-4}+6 T_{2 A}^{-3} T_{1 X}^{-1}+11 T_{2 A}^{-2} T_{1 X}^{-2}+6 T_{2 A}^{-1} T_{1 X}^{-3}+10 T_{2 A}^{-2} \pi^{2} J^{2}+30 T_{2 A}^{-1} T_{1 X}^{-1} \pi^{2} J^{2}+18 T_{1 X}^{-2} \pi^{2} J^{2}+9 \pi^{4} J^{4} \\
& =8 \frac{P+Q(i x)}{R+S(i x)}
\end{aligned}
$$

with

$$
\begin{aligned}
& P=3 x^{2}\left(T_{2 A}^{-1}+2 T_{1 X}^{-1}\right)-T_{2 A}^{-3}-6 T_{1 X}^{-1} T_{2 A}^{-2}-11 T_{1 X}^{-2} T_{2 A}^{-1}-6 T_{1 X}^{-3}-7 \pi^{2} J^{2} T_{2 A}^{-1}-15 \pi^{2} J^{2} T_{1 X}^{-1} \\
& Q=-x^{2}+3 T_{2 A}^{-2}+12 T_{1 X}^{-1} T_{2 A}^{-1}+11 T_{1 X}^{-2}+7 \pi^{2} J^{2} \\
& R=x^{4}-x^{2}\left(6 T_{2 A}^{-2}+18 T_{2 A}^{-1} T_{1 X}^{-1}+11 T_{1 X}^{-2}+10 \pi^{2} J^{2}\right) \\
& +T_{2 A}^{-4}+6 T_{2 A}^{-3} T_{1 X}^{-1}+11 T_{2 A}^{-2} T_{1 X}^{-2}+6 T_{2 A}^{-1} T_{1 X}^{-3}+10 T_{2 A}^{-2} \pi^{2} J^{2}+30 T_{2 A}^{-1} T_{1 X}^{-1} \pi^{2} J^{2}+18 T_{1 X}^{-2} \pi^{2} J^{2}+9 \pi^{4} J^{4} \\
& S=x^{2}\left(4 T_{2 A}^{-1}+6 T_{1 X}^{-1}\right)-4 T_{2 A}^{-3}-18 T_{2 A}^{-2} T_{1 X}^{-1}-22 T_{2 A}^{-1} T_{1 X}^{-2}-6 T_{1 X}^{-3}-20 T_{2 A}^{-1} \pi^{2} J^{2}-30 T_{1 X}^{-1} \pi^{2} J^{2} \\
& I(x)=8 \frac{P R+Q S x^{2}}{R^{2}+S^{2} x^{2}}(\text { eq.S4 })
\end{aligned}
$$


Approximate solution around $\mathrm{x}=0$ :

$$
\begin{aligned}
& f(0)=-\frac{T_{2 A}^{-4}+6 T_{2 A}^{-3} T_{1 X}^{-1}+11 T_{2 A}^{-2} T_{1 X}^{-2}+6 T_{2 A}^{-1} T_{1 X}^{-3}+10 T_{2 A}^{-2} \pi^{2} J^{2}+30 T_{2 A}^{-1} T_{1 X}^{-1} \pi^{2} J^{2}+18 T_{1 X}^{-2} \pi^{2} J^{2}+9 \pi^{4} J^{4}}{T_{2 A}^{-3}+6 T_{1 X}^{-1} T_{2 A}^{-2}+11 T_{1 X}^{-2} T_{2 A}^{-1}+6 T_{1 X}^{-3}+7 \pi^{2} J^{2} T_{2 A}^{-1}+15 \pi^{2} J^{2} T_{1 X}^{-1}} \\
& \left(T_{2 A}^{-3}+6 T_{1 X}^{-1} T_{2 A}^{-2}+11 T_{1 X}^{-2} T_{2 A}^{-1}+6 T_{1 X}^{-3}+7 \pi^{2} J^{2} T_{2 A}^{-1}+15 \pi^{2} J^{2} T_{1 X}^{-1}\right)\left(4 T_{2 A}^{-3}+18 T_{2 A}^{-2} T_{1 X}^{-1}+22 T_{2 A}^{-1} T_{1 X}^{-2}\right. \\
& \left.+6 T_{1 X}^{-3}+20 T_{2 A}^{-1} \pi^{2} J^{2}+30 T_{1 X}^{-1} \pi^{2} J^{2}\right)-\left(T_{2 A}^{-4}+6 T_{2 A}^{-3} T_{1 X}^{-1}+11 T_{2 A}^{-2} T_{1 X}^{-2}+6 T_{2 A}^{-1} T_{1 X}^{-3}+10 T_{2 A}^{-2} \pi^{2} J^{2}\right. \\
& f^{\prime}(0)=\frac{\left.+30 T_{2 A}^{-1} T_{1 X}^{-1} \pi^{2} J^{2}+18 T_{1 X}^{-2} \pi^{2} J^{2}+9 \pi^{4} J^{4}\right)\left(3 T_{2 A}^{-2}+12 T_{1 X}^{-1} T_{2 A}^{-1}+11 T_{1 X}^{-2}+7 \pi^{2} J^{2}\right)}{\left(T_{2 A}^{-3}+6 T_{1 X}^{-1} T_{2 A}^{-2}+11 T_{1 X}^{-2} T_{2 A}^{-1}+6 T_{1 X}^{-3}+7 \pi^{2} J^{2} T_{2 A}^{-1}+15 \pi^{2} J^{2} T_{1 X}^{-1}\right)^{2}}= \\
& 36 T_{1 X}^{-6}+132 T_{1 X}^{-5} T_{2 A}^{-1}+193 T_{1 X}^{-4} T_{2 A}^{-2}+144 T_{1 X}^{-3} T_{2 A}^{-3}+58 T_{1 X}^{-2} T_{2 A}^{-4}+12 T_{1 X}^{-1} T_{2 A}^{-5}+T_{2 A}^{-6} \\
& +72 T_{1 X}^{-4} \pi^{2} J^{2}+234 T_{1 X}^{-3} T_{2 A}^{-1} \pi^{2} J^{2}+223 T_{1 X}^{-2} T_{2 A}^{-2} \pi^{2} J^{2}+84 T_{1 X}^{-1} T_{2 A}^{-3} \pi^{2} J^{2}+11 T_{2 A}^{-4} \pi^{2} J^{2} \\
& =\frac{+225 T_{1 X}^{-2} \pi^{4} J^{4}+192 T_{1 X}^{-1} T_{2 A}^{-1} \pi^{4} J^{4}+43 T_{2 A}^{-2} \pi^{4} J^{4}-63 \pi^{6} J^{6}}{\left(T_{2 A}^{-3}+6 T_{1 X}^{-1} T_{2 A}^{-2}+11 T_{1 X}^{-2} T_{2 A}^{-1}+6 T_{1 X}^{-3}+7 \pi^{2} J^{2} T_{2 A}^{-1}+15 \pi^{2} J^{2} T_{1 X}^{-1}\right)^{2}} \\
& \Rightarrow
\end{aligned}
$$




$$
\begin{aligned}
& f(i x) \approx \text { const. }\left(i x-\frac{f(0)}{f^{\prime}(0)}\right) \approx \text { const. }(\text { ix }-\pi W) \\
& \left(T_{2 A}^{-4}+6 T_{2 A}^{-3} T_{1 X}^{-1}+11 T_{2 A}^{-2} T_{1 X}^{-2}+6 T_{2 A}^{-1} T_{1 X}^{-3}+10 T_{2 A}^{-2} \pi^{2} J^{2}+30 T_{2 A}^{-1} T_{1 X}^{-1} \pi^{2} J^{2}+18 T_{1 X}^{-2} \pi^{2} J^{2}+9 \pi^{4} J^{4}\right) \\
& \pi W \approx \frac{\left(T_{2 A}^{-3}+6 T_{1 X}^{-1} T_{2 A}^{-2}+11 T_{1 X}^{-2} T_{2 A}^{-1}+6 T_{1 X}^{-3}+7 \pi^{2} J^{2} T_{2 A}^{-1}+15 \pi^{2} J^{2} T_{1 X}^{-1}\right)}{36 T_{1 X}^{-6}+132 T_{1 X}^{-5} T_{2 A}^{-1}+193 T_{1 X}^{-4} T_{2 A}^{-2}+144 T_{1 X}^{-3} T_{2 A}^{-3}+58 T_{1 X}^{-2} T_{2 A}^{-4}+12 T_{1 X}^{-1} T_{2 A}^{-5}+T_{2 A}^{-6}}= \\
& +72 T_{1 X}^{-4} \pi^{2} J^{2}+234 T_{1 X}^{-3} T_{2 A}^{-1} \pi^{2} J^{2}+223 T_{1 X}^{-2} T_{2 A}^{-2} \pi^{2} J^{2}+84 T_{1 X}^{-1} T_{2 A}^{-3} \pi^{2} J^{2}+11 T_{2 A}^{-4} \pi^{2} J^{2} \\
& +225 T_{1 X}^{-2} \pi^{4} J^{4}+192 T_{1 X}^{-1} T_{2 A}^{-1} \pi^{4} J^{4}+43 T_{2 A}^{-2} \pi^{4} J^{4}-63 \pi^{6} J^{6} \\
& 36 T_{1 X}^{-6} T_{2 A}^{-1}+T_{1 X}^{-5}\left(132 T_{2 A}^{-2}+108 \pi^{2} J^{2}\right)+T_{1 X}^{-4}\left(193 T_{2 A}^{-3}+468 T_{2 A}^{-1} \pi^{2} J^{2}\right) \\
& +T_{1 X}^{-3}\left(144 T_{2 A}^{-4}+705 T_{2 A}^{-2} \pi^{2} J^{2}+324 \pi^{4} J^{4}\right)+T_{1 X}^{-2}\left(58 T_{2 A}^{-5}+475 T_{2 A}^{-3} \pi^{2} J^{2}+675 T_{2 A}^{-1} \pi^{4} J^{4}\right) \\
& +T_{1 X}^{-1}\left(12 T_{2 A}^{-6}+147 T_{2 A}^{-4} \pi^{2} J^{2}+414 T_{2 A}^{-2} \pi^{4} J^{4}+135 \pi^{6} J^{6}\right) \\
& =\frac{+T_{2 A}^{-7}+17 T_{2 A}^{-5} \pi^{2} J^{2}+79 T_{2 A}^{-3} \pi^{4} J^{4}+63 T_{2 A}^{-1} \pi^{6} J^{6}}{36 T_{1 X}^{-6}+132 T_{1 X}^{-5} T_{2 A}^{-1}+193 T_{1 X}^{-4} T_{2 A}^{-2}+144 T_{1 X}^{-3} T_{2 A}^{-3}+58 T_{1 X}^{-2} T_{2 A}^{-4}+12 T_{1 X}^{-1} T_{2 A}^{-5}+T_{2 A}^{-6}}= \\
& +72 T_{1 X}^{-4} \pi^{2} J^{2}+234 T_{1 X}^{-3} T_{2 A}^{-1} \pi^{2} J^{2}+223 T_{1 X}^{-2} T_{2 A}^{-2} \pi^{2} J^{2}+84 T_{1 X}^{-1} T_{2 A}^{-3} \pi^{2} J^{2}+11 T_{2 A}^{-4} \pi^{2} J^{2} \\
& +225 T_{1 X}^{-2} \pi^{4} J^{4}+192 T_{1 X}^{-1} T_{2 A}^{-1} \pi^{4} J^{4}+43 T_{2 A}^{-2} \pi^{4} J^{4}-63 \pi^{6} J^{6} \\
& 36 T_{2 A}^{-1}+T_{1 X}\left(132 T_{2 A}^{-2}+108 \pi^{2} J^{2}\right)+T_{1 X}^{2}\left(193 T_{2 A}^{-3}+468 T_{2 A}^{-1} \pi^{2} J^{2}\right) \\
& +T_{1 X}^{3}\left(144 T_{2 A}^{-4}+705 T_{2 A}^{-2} \pi^{2} J^{2}+324 \pi^{4} J^{4}\right)+T_{1 X}^{4}\left(58 T_{2 A}^{-5}+475 T_{2 A}^{-3} \pi^{2} J^{2}+675 T_{2 A}^{-1} \pi^{4} J^{4}\right) \\
& +T_{1 X}^{5}\left(12 T_{2 A}^{-6}+147 T_{2 A}^{-4} \pi^{2} J^{2}+414 T_{2 A}^{-2} \pi^{4} J^{4}+135 \pi^{6} J^{6}\right) \\
& =\frac{+T_{1 X}^{6}\left(T_{2 A}^{-7}+17 T_{2 A}^{-5} \pi^{2} J^{2}+79 T_{2 A}^{-3} \pi^{4} J^{4}+63 T_{2 A}^{-1} \pi^{6} J^{6}\right)}{36+132 T_{1 X} T_{2 A}^{-1}+T_{1 X}^{2}\left(193 T_{2 A}^{-2}+72 \pi^{2} J^{2}\right)+T_{1 X}^{3}\left(144 T_{2 A}^{-3}+234 T_{2 A}^{-1} \pi^{2} J^{2}\right)} \\
& +T_{1 X}^{4}\left(58 T_{2 A}^{-4}+223 T_{2 A}^{-2} \pi^{2} J^{2}+225 \pi^{4} J^{4}\right)+T_{1 X}^{5}\left(12 T_{2 A}^{-5}+84 T_{2 A}^{-3} \pi^{2} J^{2}+192 T_{2 A}^{-1} \pi^{4} J^{4}\right) \\
& +T_{1 X}^{6}\left(T_{2 A}^{-6}+11 T_{2 A}^{-4} \pi^{2} J^{2}+43 T_{2 A}^{-2} \pi^{4} J^{4}-63 \pi^{6} J^{6}\right) \\
& 1+12 T_{1 X}^{-1} T_{2 A}+\left(17 \pi^{2} J^{2}+58 T_{1 X}^{-2}\right) T_{2 A}^{2}+147 T_{1 X}^{-1} T_{2 A}^{3} \pi^{2} J^{2}+144 T_{1 X}^{-3} T_{2 A}^{3} \\
& +\left(79 \pi^{4} J^{4}+475 T_{1 X}^{-2} \pi^{2} J^{2}+193 T_{1 X}^{-4}\right) T_{2 A}^{4}+\left(414 T_{1 X}^{-1} \pi^{4} J^{4}+705 T_{1 X}^{-3} \pi^{2} J^{2}+132 T_{1 X}^{-5}\right) T_{2 A}^{5} \\
& +\left(468 T_{1 X}^{-4} \pi^{2} J^{2}+63 \pi^{6} J^{6}+675 T_{1 X}^{-2} \pi^{4} J^{4}+36 T_{1 X}^{-6}\right) T_{2 A}^{6} \\
& =\frac{+\left(135 T_{1 X}^{-1} \pi^{6} J^{6}+324 T_{1 X}^{-3} \pi^{4} J^{4}+108 T_{1 X}^{-5} \pi^{2} J^{2}\right) T_{2 A}^{7}}{1+12 T_{1 X}^{-1} T_{2 A}+\left(58 T_{1 X}^{-2}+11 \pi^{2} J^{2}\right) T_{2 A}^{2}+\left(144 T_{1 X}^{-3}+84 T_{1 X}^{-1} \pi^{2} J^{2}\right) T_{2 A}^{3}} T_{2 A}^{-1} \\
& +\left(43 \pi^{4} J^{4}+223 T_{1 X}^{-2} \pi^{2} J^{2}+193 T_{1 X}^{-4}\right) T_{2 A}^{4}+\left(192 T_{1 X}^{-1} \pi^{4} J^{4}+234 T_{1 X}^{-3} \pi^{2} J^{2}+132 T_{1 X}^{-5}\right) T_{2 A}^{5} \\
& +\left(72 T_{1 X}^{-4} \pi^{2} J^{2}+36 T_{1 X}^{-6}+225 T_{1 X}^{-2} \pi^{4} J^{4}-63 \pi^{6} J^{6}\right) T_{2 A}^{6}
\end{aligned}
$$




$$
\begin{aligned}
& 36 T_{1 X}^{-6} T_{2 A}^{-1}+132 T_{1 X}^{-5} T_{2 A}^{-2}+108 T_{1 X}^{-5} \pi^{2} J^{2}+193 T_{1 X}^{-4} T_{2 A}^{-3}+468 T_{1 X}^{-4} T_{2 A}^{-1} \pi^{2} J^{2} \\
& +144 T_{1 X}^{-3} T_{2 A}^{-4}+705 T_{1 X}^{-3} T_{2 A}^{-2} \pi^{2} J^{2}+324 T_{1 X}^{-3} \pi^{4} J^{4}+58 T_{1 X}^{-2} T_{2 A}^{-5}+475 T_{1 X}^{-2} T_{2 A}^{-3} \pi^{2} J^{2}+675 T_{1 X}^{-2} T_{2 A}^{-1} \pi^{4} J^{4} \\
& +12 T_{1 X}^{-1} T_{2 A}^{-6}+147 T_{1 X}^{-1} T_{2 A}^{-4} \pi^{2} J^{2}+414 T_{1 X}^{-1} T_{2 A}^{-2} \pi^{4} J^{4}+135 T_{1 X}^{-1} \pi^{6} J^{6} \\
\pi W \approx & \frac{+T_{2 A}^{-7}+17 T_{2 A}^{-5} \pi^{2} J^{2}+79 T_{2 A}^{-3} \pi^{4} J^{4}+63 T_{2 A}^{-1} \pi^{6} J^{6}}{36 T_{1 X}^{-6}+132 T_{1 X}^{-5} T_{2 A}^{-1}+193 T_{1 X}^{-4} T_{2 A}^{-2}+144 T_{1 X}^{-3} T_{2 A}^{-3}+58 T_{1 X}^{-2} T_{2 A}^{-4}+12 T_{1 X}^{-1} T_{2 A}^{-5}+T_{2 A}^{-6}} \\
& +72 T_{1 X}^{-4} \pi^{2} J^{2}+234 T_{1 X}^{-3} T_{2 A}^{-1} \pi^{2} J^{2}+223 T_{1 X}^{-2} T_{2 A}^{-2} \pi^{2} J^{2}+84 T_{1 X}^{-1} T_{2 A}^{-3} \pi^{2} J^{2}+11 T_{2 A}^{-4} \pi^{2} J^{2} \\
& +225 T_{1 X}^{-2} \pi^{4} J^{4}+192 T_{1 X}^{-1} T_{2 A}^{-1} \pi^{4} J^{4}+43 T_{2 A}^{-2} \pi^{4} J^{4}-63 \pi^{6} J^{6} \\
& \\
& 36 T_{1 X}^{-6} T_{2 A}^{-1}+132 T_{1 X}^{-5} T_{2 A}^{-2}+193 T_{1 X}^{-4} T_{2 A}^{-3}+144 T_{1 X}^{-3} T_{2 A}^{-4}+58 T_{1 X}^{-2} T_{2 A}^{-5}+12 T_{1 X}^{-1} T_{2 A}^{-6}+T_{2 A}^{-7} \\
+ & \left(108 T_{1 X}^{-5}+468 T_{1 X}^{-4} T_{2 A}^{-1}+705 T_{1 X}^{-3} T_{2 A}^{-2}+475 T_{1 X}^{-2} T_{2 A}^{-3}+147 T_{1 X}^{-1} T_{2 A}^{-4}+17 T_{2 A}^{-5}\right) \pi^{2} J^{2} \\
+ & \left(324 T_{1 X}^{-3}+675 T_{1 X}^{-2} T_{2 A}^{-1}+414 T_{1 X}^{-1} T_{2 A}^{-2}+79 T_{2 A}^{-3}\right) \pi^{4} J^{4} \\
+ & \left(135 T_{1 X}^{-1}+63 T_{2 A}^{-1}\right) \pi^{6} J^{6} \\
\hline & 36 T_{1 X}^{-6}+132 T_{1 X}^{-5} T_{2 A}^{-1}+193 T_{1 X}^{-4} T_{2 A}^{-2}+144 T_{1 X}^{-3} T_{2 A}^{-3}+58 T_{1 X}^{-2} T_{2 A}^{-4}+12 T_{1 X}^{-1} T_{2 A}^{-5}+T_{2 A}^{-6}= \\
+ & \left(72 T_{1 X}^{-4}+234 T_{1 X}^{-3} T_{2 A}^{-1}+223 T_{1 X}^{-2} T_{2 A}^{-2}+84 T_{1 X}^{-1} T_{2 A}^{-3}+11 T_{2 A}^{-4}\right) \pi^{2} J^{2} \\
+ & \left(225 T_{1 X}^{-2}+192 T_{1 X}^{-1} T_{2 A}^{-1}+43 T_{2 A}^{-2}\right) \pi^{4} J^{4}-63 \pi^{6} J^{6}
\end{aligned}
$$

Taylor expansion in $\pi^{2} J^{2}$ gives:

$$
\begin{gathered}
108 T_{1 X}^{-5}+468 T_{1 X}^{-4} T_{2 A}^{-1}+705 T_{1 X}^{-3} T_{2 A}^{-2}+475 T_{1 X}^{-2} T_{2 A}^{-3}+147 T_{1 X}^{-1} T_{2 A}^{-4}+17 T_{2 A}^{-5} \\
\pi W \approx T_{2 A}^{-1}+\frac{-72 T_{1 X}^{-4} T_{2 A}^{-1}-234 T_{1 X}^{-3} T_{2 A}^{-2}-223 T_{1 X}^{-2} T_{2 A}^{-3}-84 T_{1 X}^{-1} T_{2 A}^{-4}-11 T_{2 A}^{-5}}{36 T_{1 X}^{-6}+132 T_{1 X}^{-5} T_{2 A}^{-1}+193 T_{1 X}^{-4} T_{2 A}^{-2}+144 T_{1 X}^{-3} T_{2 A}^{-3}+58 T_{1 X}^{-2} T_{2 A}^{-4}+12 T_{1 X}^{-1} T_{2 A}^{-5}+T_{2 A}^{-6}} \pi^{2} J^{2}= \\
=T_{2 A}^{-1}+\frac{108 T_{1 X}^{-5}+396 T_{1 X}^{-4} T_{2 A}^{-1}+471 T_{1 X}^{-3} T_{2 A}^{-2}+252 T_{1 X}^{-2} T_{2 A}^{-3}+63 T_{1 X}^{-1} T_{2 A}^{-4}+6 T_{2 A}^{-5}}{36 T_{1 X}^{-6}+132 T_{1 X}^{-5} T_{2 A}^{-1}+193 T_{1 X}^{-4} T_{2 A}^{-2}+144 T_{1 X}^{-3} T_{2 A}^{-3}+58 T_{1 X}^{-2} T_{2 A}^{-4}+12 T_{1 X}^{-1} T_{2 A}^{-5}+T_{2 A}^{-6}} \pi^{2} J^{2}= \\
108\left(T_{1 X}^{-1}+T_{2 A}^{-1}\right)^{5}-144 T_{2 A}^{-1}\left(T_{1 X}^{-1}+T_{2 A}^{-1}\right)^{4}-33 T_{2 A}^{-2}\left(T_{1 X}^{-1}+T_{2 A}^{-1}\right)^{3} \\
=T_{2 A}^{-1}+\frac{+135 T_{2 A}^{-3}\left(T_{1 X}^{-1}+T_{2 A}^{-1}\right)^{2}-72 T_{2 A}^{-4}\left(T_{1 X}^{-1}+T_{2 A}^{-1}\right)+12 T_{2 A}^{-5}}{36\left(T_{1 X}^{-1}+T_{2 A}^{-1}\right)^{6}-84 T_{2 A}^{-1}\left(T_{1 X}^{-1}+T_{2 A}^{-1}\right)^{5}+73 T_{2 A}^{-2}\left(T_{1 X}^{-1}+T_{2 A}^{-1}\right)^{4}} \pi^{2} J^{2} \approx \\
-28 T_{2 A}^{-3}\left(T_{1 X}^{-1}+T_{2 A}^{-1}\right)^{3}+4 T_{2 A}^{-4}\left(T_{1 X}^{-1}+T_{2 A}^{-1}\right)^{2} \\
\approx T_{2 A}^{-1}+3 \frac{T_{1 X}^{-1}+2 T_{2 A}^{-1}}{\left(T_{1 X}^{-1}+T_{2 A}^{-1}\right)^{2}} \pi^{2} J^{2}
\end{gathered}
$$

Again, this approximation over-estimates the width. 
The least square fit of the apparent single peak to a Lorentz curve results in a peak narrower near the maximum than the actual signal. The solution above approximates the actual signal to a Lorentz peak near the peak maximum $(\mathrm{x}=0)$, thus the equations above always over-estimate the line width of the least square fit, but gives the functional shape of the expression $(n=1,2,3)$ :

$$
\pi W \approx T_{2 A}^{-1}+n \frac{T_{1 X}^{-1}+2 T_{2 A}^{-1}}{\left(T_{1 X}^{-1}+T_{2 A}^{-1}\right)^{2}} \pi^{2} J^{2}
$$

Least square fit to Lorentz peaks for the exact peak shape for the parameter ranges $\mathbf{J}$ from $3 \ldots 300 \mathrm{~Hz}, \mathrm{~T}_{1 \mathrm{X}}{ }^{-1}$ from $100 \ldots 2000 \mathrm{~Hz}$ and $\mathrm{T}_{2 \mathrm{~A}}{ }^{-1}$ from $100 \ldots 5000 \mathrm{~Hz}$ should cover all reasonable values. All data can be fitted well to the following expression with $n=1$ for $d$, $\mathrm{n}=2$ for $\mathrm{t}$ and $\mathrm{n}=3$ for $\mathrm{q}$ :

$\pi W \approx T_{2 A}^{-1}+n \frac{\pi^{2} J^{2}}{T_{1 X}^{-1}+T_{2 A}^{-1}}($ eq.4 $)$

better:

$\pi W \approx T_{2 A}^{-1}\left[1+n \frac{\pi^{2} J^{2}}{\left(T_{1 X}^{-1}+T_{2 A}^{-1}\right) T_{2 A}^{-1}}-\frac{n-1}{4 \pi}\left(\frac{\pi^{2} J^{2}}{\left(T_{1 X}^{-1}+T_{2 A}^{-1}\right) T_{2 A}^{-1}}\right)^{2}\right]($ eq.S5 $)$
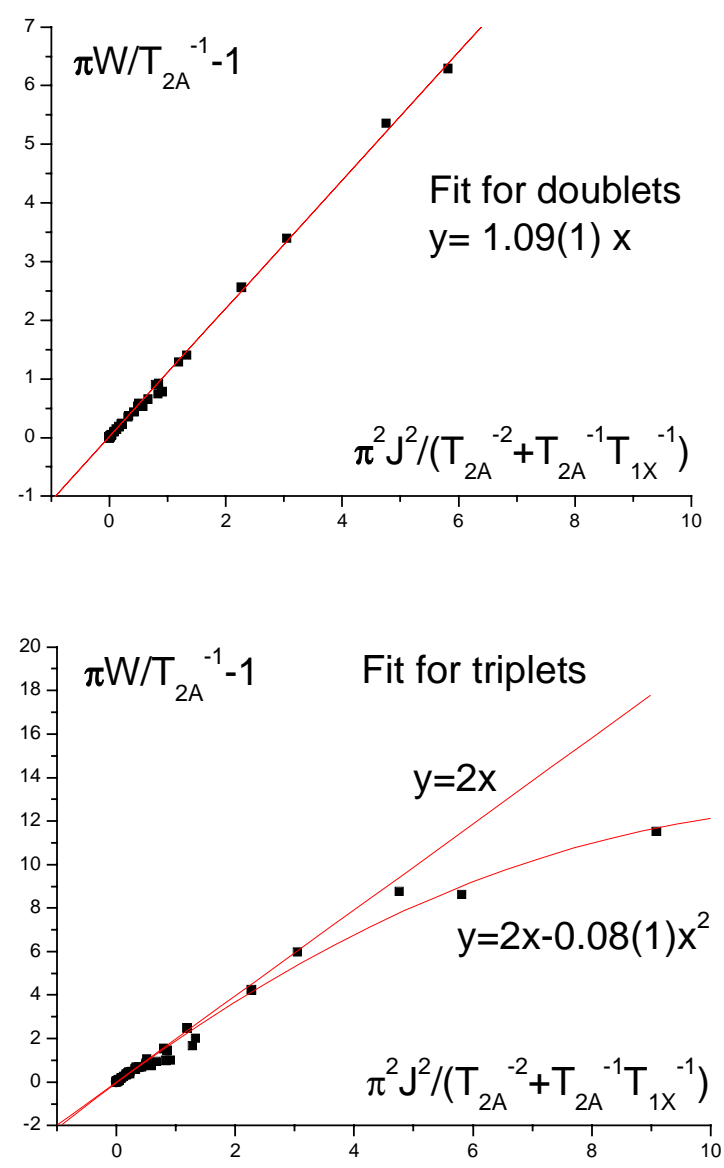

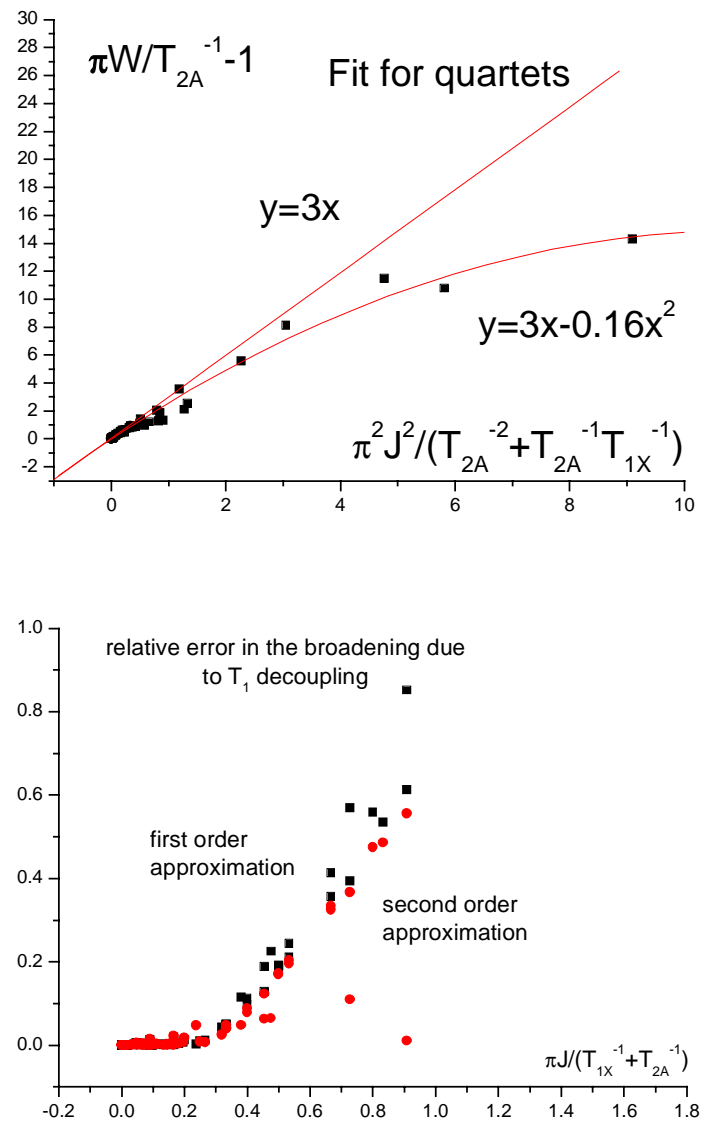

In summary, the first order approximation is good within $5 \%$ for $\mathrm{J}<\left(\mathrm{T}_{1 \mathrm{X}}{ }^{-1}+\mathrm{T}_{2 \mathrm{~A}}{ }^{-1}\right) / 10$ and the second order approximation gives only slight improvement beyond this.

The first order approximation allows to propose the following general expression for the line width of a signal A with broadening after coalescence due to $T_{1}$ spin decoupling of several spin $1 / 2$ nuclei $X$ with coupling constants $J_{X}$ and relaxation times $T_{1 X}$ :

$\pi W_{A} \approx T_{2 A}^{-1}+\sum_{X} \frac{\pi^{2} J_{X}^{2}}{T_{1 X}^{-1}+T_{2 A}^{-1}}(e q .4)$ 
Table 3: Linewidth $\mathrm{W}$ obtained from least square fitting of the curve calculated by eq.S1 (doublet), eq. S3 (triplet) and eq. S4 (quartet) to a single Lorentz peak and comparison to the approximations by eq.4 (FIT A)and eq.S5(second order)(FIT B):

\begin{tabular}{|c|c|c|c|c|c|c|c|c|c|c|}
\hline $\begin{array}{l}\pi \mathrm{J} \text { in } \\
\mathrm{Hz}\end{array}$ & $\begin{array}{l}\mathrm{T}_{2 \mathrm{~A}^{-1}} \\
\text { in } \\
\mathrm{Hz}\end{array}$ & $\begin{array}{l}T_{1 X}^{-1} \text { in } \\
\mathrm{Hz}\end{array}$ & $\mathrm{W}$ for $\mathrm{d}$ & $\begin{array}{l}\text { d: } \\
\text { FIT A }\end{array}$ & $\mathrm{W}$ for $\mathrm{t}$ & $\begin{array}{l}\text { t: } \\
\text { FIT A }\end{array}$ & $\begin{array}{l}\text { t: } \\
\text { FIT B }\end{array}$ & $\mathrm{W}$ for $\mathrm{q}$ & $\begin{array}{l}\text { q: } \\
\text { FIT A }\end{array}$ & $\begin{array}{l}\text { q: } \\
\text { FIT B }\end{array}$ \\
\hline 1000 & 1000 & 1000 & 486.7 & 477.5 & 586.5 & 636.6 & 630.3 & 669.5 & 795.8 & 783.1 \\
\hline 800 & 1000 & 1000 & 428.2 & 420.2 & 505 & 522.0 & 519.4 & 568.9 & 623.9 & 618.7 \\
\hline 500 & 1000 & 1000 & 362.1 & 358.1 & 399.7 & 397.9 & 397.5 & 433.1 & 437.7 & 436.9 \\
\hline 100 & 1000 & 1000 & 320.1 & 319.9 & 321.8 & 321.5 & 321.5 & 323.6 & 323.1 & 323.1 \\
\hline 10 & 1000 & 1000 & 318.328 & 318.3 & 318.345 & 318.3 & 318.3 & 318.363 & 318.4 & 318.4 \\
\hline-- & -- & -- & -- & -- & -- & -- & -- & -- & -- & -- \\
\hline 1000 & 500 & 1000 & 381.9 & 371.4 & 477.5 & 583.6 & 561.1 & 563.2 & 795.7 & 750.7 \\
\hline 800 & 500 & 1000 & 306.6 & 295.0 & 388.3 & 430.8 & 421.6 & 455.7 & 566.6 & 548.1 \\
\hline 500 & 500 & 1000 & 218.4 & 212.2 & 264.3 & 265.3 & 263.9 & 303.3 & 318.3 & 315.5 \\
\hline 100 & 500 & 1000 & 161.54 & 161.3 & 163.9 & 163.4 & 163.4 & 166.24 & 165.5 & 165.5 \\
\hline 10 & 500 & 1000 & 159.179 & 159.2 & 159.203 & 159.2 & 159.4 & 159.227 & 159.2 & 159.2 \\
\hline-- & -- & -- & -- & -- & -- & -- & -- & -- & -- & -- \\
\hline 1000 & 5000 & 1000 & 1647.1 & 1644.6 & 1699.1 & 1697.7 & 1697.5 & 1748.1 & 1750.7 & 1750.4 \\
\hline 800 & 5000 & 1000 & 1627.2 & 1625.5 & 1661.3 & 1659.5 & 1659.4 & 1694.1 & 1693.4 & 1693.3 \\
\hline 500 & 5000 & 1000 & 1605.5 & 1604.8 & 1619.2 & 1618.1 & 1618.1 & 1632.7 & 1631.3 & 1631.3 \\
\hline 100 & 5000 & 1000 & 1592.11 & 1592.1 & 1592.67 & 1592.6 & 1592.6 & 1593.22 & 1593.1 & 1593.1 \\
\hline 10 & 5000 & 1000 & 1591.555 & 1591.6 & 1591.561 & 1591.6 & 1591.6 & 1591.566 & 1591.6 & 1591.6 \\
\hline-- & -- & -- & -- & -- & -- & -- & -- & -- & -- & -- \\
\hline 1000 & 100 & 1000 & -- & 321.2 & 398.5 & 610.6 & 401.2 & 486.5 & 899.9 & 481.3 \\
\hline 800 & 100 & 1000 & 232 & 217.0 & 306.2 & 402.2 & 316.5 & 374.8 & 587.4 & 415.9 \\
\hline 500 & 100 & 1000 & 113.2 & 104.2 & 166.5 & 176.5 & 163.4 & 209.5 & 248.9 & 222.7 \\
\hline 100 & 100 & 1000 & 34.94 & 34.72 & 38.02 & 37.62 & 37.60 & 41.06 & 40.51 & 40.47 \\
\hline 10 & 100 & 1000 & 31.862 & 31.86 & 31.893 & 31.89 & 31.89 & 31.924 & 31.92 & 31.92 \\
\hline-- & -- & -- & -- & -- & -- & -- & -- & -- & -- & -- \\
\hline 1000 & 1000 & 500000 & 318.947 & 318.9 & 319.583 & 319.6 & 319.6 & 320.22 & 320.2 & 320.2 \\
\hline 800 & 1000 & 500000 & 318.717 & 318.7 & 319.125 & 319.1 & 319.1 & 319.532 & 319.5 & 319.5 \\
\hline 500 & 1000 & 500000 & 318.469 & 318.5 & 318.628 & 318.6 & 318.6 & 318.787 & 318.8 & 318.8 \\
\hline 100 & 1000 & 500000 & 318.316 & 318.3 & 318.323 & 318.3 & 318.3 & 318.329 & 318.3 & 318.3 \\
\hline 10 & 1000 & 500000 & 318.31 & 318.3 & 318.31 & 318.3 & 318.3 & 318.31 & 318.3 & 318.3 \\
\hline-- & -- & -- & -- & -- & -- & -- & -- & -- & -- & -- \\
\hline 1000 & 1000 & 500 & 525.3 & 530.5 & 617.8 & 742.7 & 731.5 & 704.1 & 954.9 & 932.4 \\
\hline 800 & 1000 & 500 & 455.7 & 454.1 & 533.5 & 589.9 & 585.3 & 599.8 & 725.7 & 716.5 \\
\hline 500 & 1000 & 500 & 374.2 & 371.4 & 417.5 & 424.4 & 423.7 & 454.4 & 477.5 & 476.1 \\
\hline 100 & 1000 & 500 & 320.6 & 320.4 & 322.86 & 322.6 & 322.5 & 325.1 & 324.7 & 324.7 \\
\hline 10 & 1000 & 500 & 318.333 & 318.3 & 318.356 & 318.4 & 318.4 & 318.379 & 318.4 & 318.4 \\
\hline-- & -- & -- & -- & -- & -- & -- & -- & -- & -- & -- \\
\hline 1000 & 1000 & 2000 & 436.8 & 424.4 & 528.8 & 530.5 & 527.7 & 606.6 & 636.6 & 631.0 \\
\hline 800 & 1000 & 2000 & 394.5 & 386.2 & 459 & 454.1 & 453.0 & 515.9 & 522.0 & 519.7 \\
\hline 500 & 1000 & 2000 & 348.1 & 344.8 & 376.1 & 371.4 & 371.2 & 402.4 & 397.9 & 397.5 \\
\hline 100 & 1000 & 2000 & 319.5 & 319.4 & 320.69 & 320.4 & 320.4 & 321.88 & 321.5 & 321.5 \\
\hline 10 & 1000 & 2000 & 318.322 & 318.3 & 318.334 & 318.3 & 318.3 & 318.346 & 318.3 & 318.3 \\
\hline-- & -- & -- & -- & -- & -- & -- & -- & -- & -- & -- \\
\hline 1000 & 1000 & 100 & 565.7 & 607.7 & 637.1 & 897.1 & 876.1 & 735.9 & 1186.4 & 1144.6 \\
\hline 800 & 1000 & 100 & 486.1 & 503.5 & 555.3 & 688.7 & 680.1 & 627.1 & 873.9 & 856.8 \\
\hline 500 & 1000 & 100 & 388.7 & 390.7 & 434.9 & 463.0 & 461.7 & 474.3 & 535.3 & 532.7 \\
\hline 100 & 1000 & 100 & 321.26 & 321.2 & 324.16 & 324.1 & 324.1 & 326.99 & 327.0 & 327.0 \\
\hline 10 & 1000 & 100 & 318.339 & 318.3 & 318.369 & 318.4 & 318.4 & 318.399 & 318.4 & 318.4 \\
\hline-- & -- & -- & -- & -- & -- & -- & -- & -- & -- & -- \\
\hline 800 & 500 & 500 & -- & 362.9 & 423.3 & 566.6 & 545.8 & 494.5 & 770.3 & 728.8 \\
\hline 500 & 500 & 500 & 243.3 & 238.7 & 293.2 & 318.3 & 315.1 & 334.6 & 397.9 & 391.6 \\
\hline 100 & 500 & 500 & 162.69 & 162.3 & 166.13 & 165.5 & 165.5 & 169.49 & 168.7 & 168.7 \\
\hline 10 & 500 & 500 & 159.19 & 159.9 & 159.226 & 159.2 & 159.2 & 159.261 & 159.3 & 159.3 \\
\hline-- & -- & -- & -- & -- & -- & -- & -- & -- & -- & -- \\
\hline 1000 & 500 & 2000 & 302.2 & 286.5 & 403.5 & 413.8 & 405.7 & 486.7 & 541.1 & 524.9 \\
\hline 800 & 500 & 2000 & 250.8 & 240.6 & 324.3 & 322.1 & 318.8 & 387.1 & 403.6 & 397.0 \\
\hline 500 & 500 & 2000 & 194.8 & 191.0 & 227.6 & 222.8 & 222.3 & 258.1 & 254.6 & 253.0 \\
\hline 100 & 500 & 2000 & 160.57 & 160.4 & 161.98 & 161.7 & 161.7 & 163.39 & 163.0 & 163.0 \\
\hline 10 & 500 & 2000 & 159.169 & 159.2 & 159.183 & 159.2 & 159.2 & 159.197 & 159.2 & 159.2 \\
\hline-- & -- & -- & -- & -- & -- & -- & -- & -- & -- & -- \\
\hline
\end{tabular}


S40

\begin{tabular}{|c|c|c|c|c|c|c|c|c|c|c|}
\hline 1000 & 500 & 100 & -- & 689.7 & -- & 1220.2 & 1079.5 & -- & 1750.7 & 1469.3 \\
\hline 800 & 500 & 100 & -- & 498.7 & -- & 838.2 & 780.6 & -- & 1177.7 & 1062.5 \\
\hline 500 & 500 & 100 & 277.3 & 291.8 & 316.6 & 424.4 & 415.6 & 363.4 & 557.0 & 539.5 \\
\hline 100 & 500 & 100 & 164.64 & 164.5 & 169.76 & 169.8 & 169.8 & 174.59 & 175.1 & 175.0 \\
\hline 10 & 500 & 100 & 159.21 & 159.2 & 159.265 & 159.3 & 159.3 & 159.321 & 159.3 & 159.3 \\
\hline-- & -- & -- & -- & -- & -- & -- & -- & -- & -- & -- \\
\hline 1000 & 5000 & 500 & 1651 & 1649.4 & 1706 & 1707.3 & 1707.1 & 1757.4 & 1765.2 & 1764.8 \\
\hline 800 & 5000 & 500 & 1629.7 & 1628.5 & 1665.9 & 1665.6 & 1665.6 & 1700.6 & 1702.7 & 1702.5 \\
\hline 500 & 5000 & 500 & 1606.5 & 1606.0 & 1621.1 & 1620.5 & 1620.5 & 1635.5 & 1635.0 & 1634.9 \\
\hline 100 & 5000 & 500 & 1592.15 & 1592.1 & 1592.75 & 1592.7 & 1592.7 & 1593.34 & 1593.3 & 1593.3 \\
\hline 10 & 5000 & 500 & 1591.555 & 1591.6 & 1591.561 & 1591.6 & 1591.6 & 1591.567 & 1591.6 & 1591.6 \\
\hline-- & -- & -- & -- & -- & -- & -- & -- & -- & -- & -- \\
\hline 1000 & 5000 & 2000 & 1640.5 & 1637.0 & 1687.1 & 1682.5 & 1682.4 & 1731.7 & 1728.0 & 1727.8 \\
\hline 800 & 5000 & 2000 & 1622.9 & 1620.7 & 1653.3 & 1649.8 & 1649.7 & 1682.8 & 1678.9 & 1678.8 \\
\hline 500 & 5000 & 2000 & 1603.83 & 1602.9 & 1615.96 & 1614.3 & 1614.3 & 1627.94 & 1625.7 & 1625.6 \\
\hline 100 & 5000 & 2000 & 1592.041 & 1592.0 & 1592.533 & 1592.5 & 1592.5 & 1593.024 & 1592.9 & 1592.9 \\
\hline 10 & 5000 & 2000 & 1591.554 & 1591.6 & 1591.559 & 1591.6 & 1591.6 & 1591.564 & 1591.6 & 1591.6 \\
\hline-- & -- & -- & -- & -- & -- & -- & -- & -- & -- & -- \\
\hline 1000 & 5000 & 100 & 1654.5 & 1654.0 & 1712 & 1716.4 & 1716.2 & 1765.5 & 1778.8 & 1778.4 \\
\hline 800 & 5000 & 100 & 1631.9 & 1631.5 & 1670 & 1671.4 & 1671.4 & 1706.2 & 1711.4 & 1711.2 \\
\hline 500 & 5000 & 100 & 1607.38 & 1607.2 & 1622.81 & 1622.8 & 1622.7 & 1637.97 & 1638.4 & 1638.3 \\
\hline 100 & 5000 & 100 & 1592.184 & 1592.2 & 1592.818 & 1592.8 & 1592.8 & 1593.451 & 1593.4 & 1593.4 \\
\hline 10 & 5000 & 100 & 1591.556 & 1591.6 & 1591.562 & 1591.6 & 1591.6 & 1591.568 & 1591.6 & 1591.6 \\
\hline-- & -- & -- & -- & -- & -- & -- & -- & -- & -- & -- \\
\hline 1000 & 100 & 2000 & 202.3 & 183.4 & 310.5 & 335.0 & 277.5 & 397.3 & 486.6 & 371.7 \\
\hline 800 & 100 & 2000 & 139.8 & 128.8 & 222 & 225.8 & 202.3 & 289.8 & 322.9 & 275.8 \\
\hline 500 & 100 & 2000 & 72.71 & 69.73 & 110.45 & 107.6 & 104.0 & 145.11 & 145.5 & 138.3 \\
\hline 100 & 100 & 2000 & 33.412 & 33.35 & 34.991 & 34.86 & 34.86 & 36.567 & 36.38 & 36.37 \\
\hline 10 & 100 & 2000 & 31.847 & 31.85 & 31.863 & 31.86 & 31.86 & 31.878 & 31.88 & 31.88 \\
\hline-- & -- & -- & -- & -- & -- & -- & -- & -- & -- & -- \\
\hline 100 & 100 & 500 & 37.73 & 37.14 & 43.32 & 42.44 & 42.37 & 48.64 & 47.75 & 47.61 \\
\hline 10 & 100 & 500 & 31.89 & 31.88 & 31.948 & 31.94 & 31.94 & 32.007 & 32.00 & 32.00 \\
\hline-- & -- & -- & -- & -- & -- & -- & -- & -- & -- & -- \\
\hline 100 & 100 & 100 & 48.674 & 47.75 & 58.641 & 63.66 & 63.03 & 66.921 & 79.58 & 78.31 \\
\hline 10 & 100 & 100 & 32.011 & 31.99 & 32.19 & 32.15 & 31.94 & 32.367 & 32.31 & 32.31 \\
\hline
\end{tabular}

